\title{
NUCLEAR SPECTROSCOPIC STUDIES
}

\author{
C.R. Bingham, M.W. GuidRy, L.L. Riedinger, \\ AND S.P. SORENSEN
}

\author{
Department of Physics and Astronomy \\ University of Tennessee \\ Knoxville, TN 37996-1200
}

February 18, 1994

DISCLAIMER

\begin{abstract}
This report was prepared as an account of work sponsored by an agency of the United States Government. Neither the United States Government nor any agency thereof, nor any of their employees, makes any warranty, express or implied, or assumes any legal liability or responsibility for the accuracy, completeness, or usefulness of any information, apparatus, product, or process disclosed, or represents that its use would not infringe privately owned rights. Reference herein to any specific commercial product, process, or service by trade name, trademark, manufacturer, or otherwise does not necessarily constitute or imply its endorsement, recommendation, or favoring by the United States Government or any agency thereof. The views and opinions of authors expressed herein do not necessarily state or reflect those of the United States Government or any agency thereof.
\end{abstract}

Research supported by the United States Department of Energy under contract DE-FG05-87ER40361 June 1, 1.992 to May 31, 1993. 


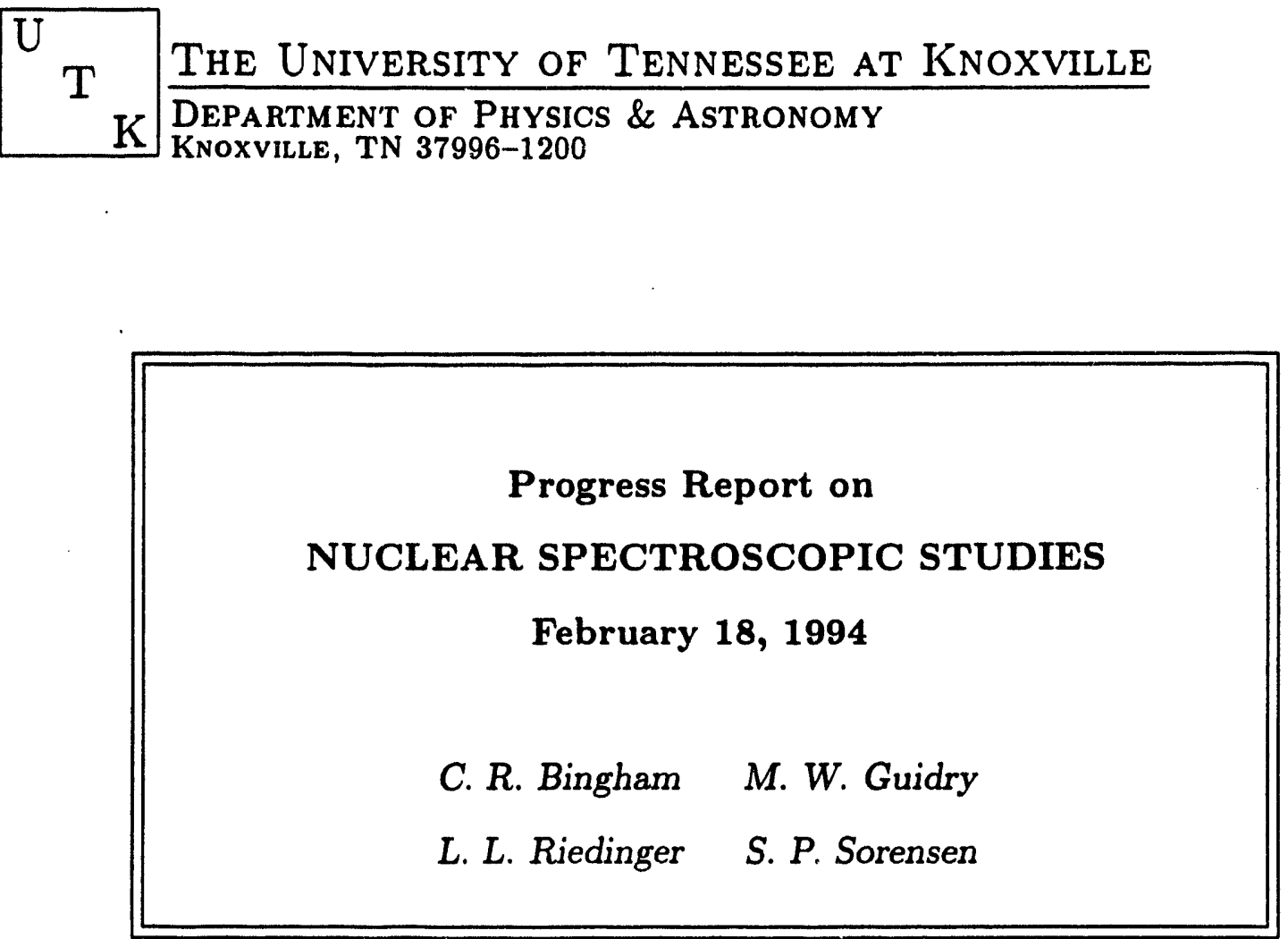

Document no. 94-01

$\mathcal{N}$ Nuclear Physics Group Document

$\mathcal{P}$ Research Supported by the U. S. Dept. of Energy 


\section{CONTENTS}

I. OVERVIEW 3

II. SCIENTIFIC RESUltS

II.B. Properties of High-Spin States . . . . . . . . . . . . . . 11

II.A.1. Prolate Collectivity in ${ }^{187} \mathrm{Tl}$. . . . . . . . . . . . . . 13

II.A.2. Search for "Normal" and Superdeformed Bands in ${ }^{189} \mathrm{Tl} . \quad$. 17

II.A.3. Multiple Band Structures in ${ }^{191} \mathrm{Tl} \ldots . . . . . . . . .20$

II.A.4. Level Lifetimes in $\pi i_{13 / 2}$ and $\pi h_{9 / 2}$ Bands of ${ }^{181} \mathrm{Ir}$ and ${ }^{187} \mathrm{Au} .23$

II.A.5. New Results on High Spin States in ${ }^{181} \mathrm{Au}$. . . . . . . . . 27

II.A.6. High Spin Studies in ${ }^{164} \mathrm{Tm}$. . . . . . . . . . . . . . . . 29

II.A.7. Lifetime Measurement for the Superdeformed Band in ${ }^{196} \mathrm{~Pb} . \quad 30$

II.A.8. Calculated Signature Splitting in W isotopes . . . . . . . 32

II.A.9. Identical Transitions between Superdeformed and Normally Deformed Bands and the Cancellation Picture. . . . . . . 34

II.A.10. Relation of Collective Observables to Nuclear Shapes. . . 36

II.B. STUdy OF LOW-Energy Levels OF NUClei FAR From STABILITY .................... 41

II.B.1. Observation of Fine Structure in ${ }^{190} \mathrm{~Pb} \alpha$ Decay . . . . . . 43

II.B.2. Alpha Decay Studies in $\mathrm{Hg}, \mathrm{Au}, \mathrm{PtNuclides}$ Using a DoubleSided Silicon Strip Detector . . . . . . . . . . . . . 47

II.B.3. Proton Radioactivity Measurements on ${ }^{146,147} \mathrm{Tm}$ and ${ }^{160} \operatorname{Re} \quad 52$

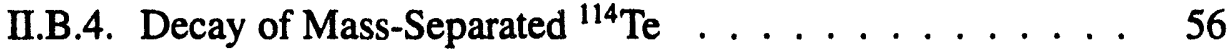

III.B.5. Decay of Mass-Separated ${ }^{199} \mathrm{Bi}$ and ${ }^{193} \mathrm{Bi} \ldots \ldots$. . . . . . 59

II.B.6. Search for $I=0$ Superdeformed Isomers in Non-actinide Nuclides: ${ }^{194} \mathrm{Hg}$. . . . . . . . . . . . . . 60

II.C. High ENERgy HeAvy-Ion Physics . . . . . . . . . . . . 65

II.C.1. Overview of the PHENIX Project . . . . . . . . . . . 67

II.C.2. Muon Detection in PHENIX . . . . . . . . . . . . . 71

II.C.3. The Off-line Computing System of PHENIX . . . . . . . 81

II.C.4. The RD93 $\mu$ ID Project . . . . . . . . . . . . . . . . . . . 85

II.C.5. Status of Data Analysis of the $\mu$ ID R\&D Project . . . . . 88

II.C.6. LabVIEW Based Small Data Acquisition System . . . . . 92

II.C.7. RD93 Data Acquisition System . . . . . . . . . . . . . . 94

II.C.8. Overview of WA80 Progress . . . . . . . . . . . . . . . 95

II.C.9. Status Report of WA80 Proton Data Analysis . . . . . . . 98

II.C.10. Azimuthal asymmetry of pion emission in proton-nucleus reactions at $200 \mathrm{GeV} \ldots \ldots \ldots 0$

II.C.11.Particle Spectra from Ultrarelativistic Collisions at Backward Angles . . . . . . . . . . . . . . 102 
II.C.12. Overview of WA93 Progress . . . . . . . . . . . . 105

II.C.13. Overview of WA98 Progress . . . . . . . . . . . . . 106

II.C.14. The Photon Spectrometer in the WA98 Experiment . . . . 106

III.The JoInt Institute for Heavy Ion RESEARCH 111

$\begin{array}{ll}\text { IV. List of Publications } & 117\end{array}$

IV.A. Papers Published or Ready for Submission . . . . . . . . . . . . . 119

IV.B. Abstracts at Meetings . . . . . . . . . . . . . . . . . 125

IV.C. Invited Talks . . . . . . . . . . . . . . . . . . 126

IV.D. Colioquia . . . . . . . . . . . . . . . . . . . . 126

IV.B. Nuclear Physics Group Seminars . . . . . . . . . . . . . . . . . . 127 



\section{Overview}

The Nuclear Physics group at the University of Tennessee, Knoxville (UTK) is involved in several aspects of heavy-ion physics including both nuclear structure and reaction mechanisms. While our main emphasis is on experimental problems, we have maintained a strong collaboration with several theorists in order to best pursue the physics of our measurements. During the last year we have had several experiments at the the ATLAS at Argonne National Laboratory, the GAMMASPHERE at the LBL 88" Cyclotron, and with the NORDBALL at the Niels Bohr Institute Tandem. Also, we continue to be very active in the WA93/98 collaboration studying ultra-relativistic heavy ion physics utilizing the SPS accelerator at CERN in Geneva, Switzerland and in the PHENIX Collaboration at the RHIC accelerator under construction at Brookhaven National Laboratory.

During the last year our experimental work has been in three broad areas: (1) the structure of nuclei at high angular momentum, (2) the structure of nuclei far from stability, and (3) ultra-relativistic heavy-ion physics. The results of studies in these particular areas will be described in this document in sections $\amalg A, ~ \Pi B$, and IIC, respectively. These studies concentrate on the structure of nuclear matter in extreme conditions of rotational motion, imbalance of neutrons and protons, or very high temperature and density. Another area of our research is heavy-ion-induced transfer reactions, which utilize the transfer of nucleons to states with high angular momentum to learn about their structure and to understand the transfer of particles, energy, and angular momentum in collisions between heavy ions.

An important component of our work is theoretical interpretation of the experimental results. This part of the work profits greatly from the Joint Institute for Heavy Ion Research (JIHIR) which is discussed in chapter III. The JIHIR has been a huge success, and greatly benefits our own research program but also the other research programs based at Oak Ridge through the flow of excellent visitors to the area and in the sponsorship of workshops and symposia which are of direct interest to our work.

The lists of publications, abstracts, and invited talks are given in chapter IV. In the past year, the research supported by the DOE contract has resulted in the publication of 29 papers; with 7 others accepted and another 10 under review or ready for submission to journals. Seven abstracts were submitted to meetings, and 11 additional invited talks, papers, or lectures were presented.

The research described here is led by four full-time UTK faculty in the Department of Physics, Carrol Bingham (Prof.), Mike Guidry (Prof.), Lee Riedinger (Prof.), 
and Soren Sorensen (Assoc. Prof.). J-y. Zhang, a theorist from Lanzhou (China) has been with us this year. In addition, several JIHIR visitors have contributed to the theoretical effort (e.g. Witek Nazarewicz) and to the experimental effort (e.g. P. Woods). Our experimental programs depend heavily on collaborations with experimentalists from ORNL, University of Lund, University of Munster, GSI, Georgia State University, Vanderbilt University, Los Alamos, VECC (Calcutta), UNISOR, LSU, Georgia Tech. University of Maryland, Lawrence Livermore Laboratory, and Argonne National Laboratory. In view of the large number of collaborations in our work, we list the actual participants at the beginning of each report in this document, execpt for the PHENIX project, which involves close to 400 scientists.

Postdoctoral research associates and graduate students play a large role in the research described here. Currently, there are three postdoctoral research associates contributing to this research: Xiaochun He in the ultra-relativistic heavy ion physics program, Walter Reviol in the program to study high spin states, and B. E. Zimmerman in the program to study nuclei far from stability. X.C. He has been responsible for the analysis of proton induced data from CERN and the implementation of a data aquisition system from the RD93 project at BNL. Zimmerman is taking the lead on experiments utilizing recoil mass separators at other facilities and is contributing to the design of detection techniques to be used with the new facility in Oak Ridge. Reviol is providing leadership on the study of high-spin states via in-beam $\gamma$-ray spectroscopy, and will devote increasing time toward development of specialized detectors to be used with arrays at Oak Ridge and Berkeley. At the present time we have seven graduate students working on some aspect of this research. M. Zhang, and X. Wang are supported at the moment fully by the DOE grant. J. Kreke, J. Lewis, J. D. Richards, B. Smith and W. Mueller receive partial support from the contract and partial support from other university funds.

Our high spin program of the last three years has been devoted primarily to a study of "intruder states" of large individual angular momentum $(j)$ and their effects at high angular momentum, both at normal and super deformation. Intruder states emanate from a position high above the Fermi level, are strongly downsloping with deformation in the usual single-particle scheme, and drop to low excitation energy by virtue of a drive of the nucleus to larger deformation. Our work has concentrated on odd- $Z$ nuclei and the deformation-driving effects of proton states, especially $h_{9 / 2}$ and $i_{13 / 2}$. In the past year, we have taken the lead in performing three major experiments at three different accelerators, and also worked on several other measurements as collaborators. The three experiments have been on high-spin states in ${ }^{187} \mathrm{Tl}$ at Argonne (the Ge-BGO array), ${ }^{164} \mathrm{Tm}$ at the Niels Bohr Institute (NORDBALL), and ${ }^{189} \mathrm{Tl}$ at Berkeley (GAMMASPHERE). 
Our work has shown that the signature splitting (in energy) between the two components of a rotational band built on a high- $j$ orbital is an indicator of the deformation. We have in press a major work (experimental and theoretical) on the systematics of the $\nu i_{13 / 2}$ signature splitting throughout the region of rare-earth deformed nuclei, and in preparation a companion paper for $\pi h_{9 / 2}$. These two studies have enabled us to extract important physics from the very complicated odd-odd nuclei that we have studied in the last few years, especially ${ }^{178} \mathrm{Ir}$, ${ }^{180} \mathrm{Ir}$, and ${ }^{164} \mathrm{Tm}$. Coupling of the "intruder" $\pi h_{9 / 2}$ orbital with $\nu i_{13 / 2}$ to form such a band in odd-odd nuclei produces a significant decrease of the characteristic $\nu i_{13 / 2}$ splitting in ${ }^{164} \mathrm{Tm}$ (since the $\pi h_{9 / 2}$ orbital is deformation driving) but not in ${ }^{178} \mathrm{Ir}$ and ${ }^{180} \mathrm{Ir}$ (since the $\pi h_{9 / 2}$ deformation drive is absent). In this way, we can use signature splitting (which we have systematized) to help make the always difficult configuration assignments in odd-odd nuclei.

We have performed experiments on two isotopes of thallium, ${ }^{187,189} \mathrm{Tl}$, in the past year, and progressed greatly on finalizing the level scheme of ${ }^{191} \mathrm{Tl}$ from our earlier experiment. The ground state and lowest configurations of these nuclei are slightly oblate in shape, but an issue for some years is locating the couplings of the high- $j$ states, $\pi h_{9 / 2}$ and $\pi i_{13 / 2}$ to the rather low lying strongly prolate excitation in the $\mathrm{Hg}$ core nuclei. Our recent measurement on ${ }^{187} \mathrm{Tl}$ at Argonne has demonstrated for the first time the existence of both of these prolate bands, coexisting with lower lying oblate bands. The earlier measurement on ${ }^{189} \mathrm{Tl}$ in France has proposed the prolate $\pi i_{13 / 2}$ band, but indicated that the prolate $\pi h_{9 / 2}$ band was absent, for a configuration blocking reason. To test this, we have taken data recently at GAMMASPHERE on ${ }^{189} \mathrm{Tl}$, to search for both these prolate bands.

Our program has encompassed also the study of superdeformed structures in Tl nuclei. We have in press a paper on the discovery of two superdeformed bands in ${ }^{191} \mathrm{Tl}$, and our recent GAMMASPHERE run on ${ }^{189} \mathrm{Tl}$ was intended partially to search for the two expected bands. The two superdeformed structures seen in odd- $A \mathrm{Tl}$ nuclei are $\pi i_{13 / 2}$ in nature (5/2[642]), and so our work on signature splitting at normal deformation has direct application to these issues at superdeformation. We are searching the new data for superdeformed bands in ${ }^{189} \mathrm{Tl}$, which would represent the lowest $N$ in this region for which such bands have been found. Our hope is to see the linking transitions between the two signatures, so that we might measure for the first time the real signature splitting in this orbital at superdeformed values.

Our measurements of rotational bands built on upsloping and downsloping orbitals has led us to an important study of identical bands. Our earlier Physical Review Letter suggested that the identical nature of bands in odd-proton nuclei throughout the rare-earth region results from simply a cancellation of two effects, 
the increase of the moment of inertia compared to adjacent even-even nuclei due to the lower pairing correlations in the odd- $Z$ nucleus and the decrease of the moment due to the smaller $\beta_{2}$ of the nucleus in the upsloping orbital. These ideas were explored further in the past year by comparing normal and superdeformed bands.

No new experiments have been done in the heavy-ion transfer program for the past year because of the decision to convert Holifield to a Radioactive Ion Beam facility. Nevertheless, over the past year we have written four papers associated with the analysis of previously acquired data. These papers deal with possible evidence for a nuclear Josephson effect, anomalous population and structure observed in the 2-8 MeV excitation energy range, measurement of the probability of populating rotational states as a angular momentum in one and two-neutron transfer, and a search for a nuclear Berry phase effect (diabolical transfer). Most importantly, they establish the foundation for next-generation experiments that that we shall propose for the new multidetector arrays and radioactive ion beam accelerators. We await the full implementation of GAMMASPHERE for our first round of such proposals.

In the area of nuclei far from stability, experimentation has continued at the Fragment Mass Analyzer on the ATLAS at Argonne National Laboratory in the study of $\alpha$ decay, and a new program to study proton radioactivity has been started in collaboration with Cary Davids, et al. Experiments included an $\alpha$ decay experiment for nuclei around ${ }^{180} \mathrm{~Pb}$, and another to find the fine structure peak corresponding to the decay of ${ }^{192} \mathrm{Po}$ to the $0_{2}^{+}$state of ${ }^{188} \mathrm{~Pb}$, which is expected to be deformed. In re-analysis of $\alpha$-decay data of ${ }^{190} \mathrm{~Pb}$ taken at UNISOR, the fine structure line corresponding to the transition to the $2_{1}^{+}$was discovered; its strength indicates that the transition is hindered, but much less so than for the the same transition in ${ }^{186.188} \mathrm{~Pb}$ decay. Three initial runs to study proton radioactivity was devoted to measurements in ${ }^{146,147} \mathrm{Tm}$ and ${ }^{160} \mathrm{Re}$.

Work has continued on the study of the low-lying single particle states and shape coexistence in the odd- $\mathrm{A} \mathrm{Pb}$ isotopes. Re-analysis of the ${ }^{199} \mathrm{Bi}$ decay data from UNISOR has uncovered an excellent candidate for the low-lying $p_{1 / 2}$ state in ${ }^{199} \mathrm{~Pb}$ at $216 \mathrm{keV}$ excitation, and the analysis of new decay data from ${ }^{193} \mathrm{Bi}$ has resulted in a scheme of about 30 previously unknown states, and again includes the probable $p_{1 / 2}$ state at $354 \mathrm{keV}$. Progress was also made in the analysis of ${ }^{114} \mathrm{Te}$ decay data which sheds light on shape coexistence properties in the region above the $Z=50$ closed shell.

The observation of superdeformed states in the high spin region has prompted several theoretical predictions relating to superdeformed nuclei. It has been predicted that the low-spin states of such superdeformed nuclei in the light $\mathrm{Hg}$ - $\mathrm{Pb}$-Po region fall at an excitation energy of perhaps 3-5 MeV. During the last years of HHIRF 
operation, we collaborated in several studies to search for low-spin members of SD bands populated via radioactive decay. Two papers concerning these searches including very small upper limits for the branch of the beta decay to the $8^{+}, 10^{+}$ and $12^{+}$members of the SD band in ${ }^{194} \mathrm{~Pb}$ were published in 1993. During the last year an experiment was carried out at the Indiana Cyclotron in collaboration with Univ. of Maryland, UNISOR, LSU and Indiana to search for short-lived isomers in ${ }^{194} \mathrm{Hg}$ populated via the $(\mathrm{p}, 4 \mathrm{n})$ reaction at $45 \mathrm{MeV}$. Also, plans were made for an experiment utilizing the isotope separator ISOLDE at CERN to observe the low-spin SD band members in ${ }^{198} \mathrm{Po},{ }^{194} \mathrm{~Pb}$, or ${ }^{190} \mathrm{Hg}$. Our first run at ISOLDE is currently being planned to occur early this year (1994).

Our research within the field of high energy nuclear physics is concentrated within two large collaborations: the WA80/93/98 collaboration at the SPS accelerator at CERN in Switzerland and the PHENIX collaboration at the new RHIC accelerator under construction at Brookhaven National Laboratory. Working within two collaborations, where one is actively taking data (WA93/98) and the other (PHENIX) is in the design/construction phase, enables us to participate in all aspects of experimental work within high energy nuclear physics.

As a continuation of our longstanding involvement with the WA80/93/98 calorimeters we spent this summer overhauling the detectors: recalibrating all modules and troubleshooting all channels as preparation for the $\mathrm{Pb}$-beam experiments in November 1994. This will be the first chance to investigate real heavy ion collisons at $160 \mathrm{GeV} /$ nucleon. We will, as usual, be in charge of the hadron calorimeters, but will also provide support for the large electromagnetic calorimeters and the subsequent data analysis. We have continued the analysis of the older proton induced data and a paper describing these data is now circulating within the collaboration for comments.

Our main focus this years has, however, been work related to PHENIX. In January Dr. Sorensen was appointed to the Detector Council of PHENIX with responsibilty for the design and construction of the off-line computer system. The requirements to this system will make it into one of the most powerful scientific computer systems: it will need 50-100 GFlops of CPU power and will have to be able to process 500 TeraBytes of data each year. In addition to the offline work we have been active with the design of the muon arm in PHENIX. In 1993 the muon arm had to go through three major DOE mandated reviews. In each case we prepared crucial material. In particular our work on tracking in the muon arm was important, since we were able to demonstrate through the creation of a set of advanced algorithms, that tracking of muons at the $90-95 \%$ efficiency level is possible even in the very "dirty" background environment created especially by leakage from the two 
magnets in PHENIX. In the muon absorber R\&D project we have taken a major role in the data aquisition and data analysis.

The Nuclear Physics programs at the University of Tennessee and at the Oak Ridge National Laboratory continue to receive considerable support from the university and the state. Most of this support comes from the state through the Science Alliance, which is the first and largest of the Centers of Excellence funded by the State of Tennessee. The Science Alliance has supported the nuclear physics effort at UTK/ORNL in a variety of ways, including partial support of Holifield equipment purchases (Compton-suppressed Ge detectors, dilution refrigerator, and the Recoil Mass Spectrometer), partial support of graduate students in our group, undergraduate summer research appointments, support of our effort in the relativistic heavy-ion program at CERN, and the major part of the operating fund for the Joint Institute for Heavy Ion Research. Our group has contributed much to the operation of the JIHIR, but has also benefited from the visitors to the institute. The JIHIR is administered through UTK, which means that all of the visitors are paid and processed through our university offices. There have been a total of 56 visitors to the Joint Institute in the past year working in support of Holifield programs, some involving our group; the average length of these appointments was approximately 3 months. While a significant portion of the JIHIR budget was spent in support of nuclear theory relevant to RIB physics, another large fraction was spent in support of RIB, RMS, and detector development for the new HRIBF under construction. The JIHIR has had a major impact on the vitality of nuclear physics in the state of Tennessee, and on the health of the field at an international level.

Concerning committee work in the community, Lee Riedinger was recently elected to be the vice chair of the Division of Nuclear Physics (DNP) of the American Physical Society, and continues to serve as chairman of the Nuclear Science Resources Committee in the DNP. At the University of Tennessee, he presently holds the position of Associate Vice Chancellor for Research on an acting basis. Carrol Bingham serves as vice chair of the UNISOR executive committee and as chairman of its Technical Committee, is a council member of the Joint Institute for Heavy Ion Research and is an Executive Council Member of the Southeastern Section of the American Physical Society. Soren Sorensen is a member of the Detector Council for the PHENIX Collaboration at Brookhaven National Laboratory and is also serving as the PHENIX representative in the planning committee for the RHIC Computing Center. 


\section{Scientific Results}




\section{II.A. Properties of High-Spin States}




\section{II.A.1. Prolate Collectivity in ${ }^{187} \mathbf{T l}$.}

W. Reviol, L.L. Riedinger, J.-Y. Zhang, C.R. Bingham, W.F. Mueller, B.E. Zimmerman [UTK]; R.V.F. Janssens, M.P. Carpenter, I. Ahmad, I.G. Bearden, R.G. Henry, T.L. Khoo, T. Lauritsen, Y. Liang [ANL].

Light mercury and lead nuclei exhibit a variety of shapes, ranging from a slightly oblate ground state $\left(\left|\epsilon_{2}\right| \approx 0.14\right)$ to a prolate intruder band of rotational states with rather strong deformation $\left(\left|\epsilon_{2}\right| \approx 0.22\right)$, and even to a superdeformed prolate structure $\left(\left|\epsilon_{2}\right| \approx 0.45\right)[1]$. The purpose of this work was to explore the microscopic structure of this low-lying prolate minimum (e.g. $E\left(0_{2}^{+}\right)=523 \mathrm{keV}$ in ${ }^{186} \mathrm{Hg}$ [1] and $E\left(0_{2}^{+}\right) \approx 720 \mathrm{keV}$ in $\left.{ }^{188} \mathrm{~Pb}[2,3]\right)$ by searching for the occurrence of prolate $h_{9 / 2}$ and $i_{13 / 2}$ rotational bands in an odd $Z$ neighbor, ${ }^{187} \mathrm{Tl}$, an isotone of both ${ }^{186} \mathrm{Hg}$ and ${ }^{188} \mathrm{~Pb}$.

Both the $h_{9 / 2}$ and $i_{13 / 2}$ orbitals are expected to play a major role in the formation of the excited prolate minimum in light $\mathrm{Hg}$ and $\mathrm{Pb}$ nuclei due to their strongly "downsloping" character. In fact, based on the observation of a prolate $\pi i_{13 / 2}$ band in ${ }^{189} \mathrm{Tl}$, and the apparent lack of feeding of a prolate $\pi h_{9 / 2}$ structure, Porquet et al. [4] argued that the prolate $\mathrm{Hg}$ core could be largely of $\left(\pi h_{9 / 2}\right)^{2}$ character. Our experiment and calculations on ${ }^{187} \mathrm{Tl}$ test this "blocking" hypothesis in a case where the prolate minimum is even lower in energy in the core nuclei, ${ }^{186} \mathrm{Hg}$ and ${ }^{188} \mathrm{~Pb}$. We find that prolate bands based on both $\pi h_{9 / 2}$ and $\pi i_{13 / 2}$ orbitals are present in ${ }^{187} \mathrm{Tl}$ and describe calculations which analyze the role of both of these high- $j$ proton orbitals in the formation of the prolate minimum. A publication of these results has been accepted by Physical Review [5].

The experiment was performed at the ATLAS accelerator at ANL. The states in ${ }^{187} \mathrm{Tl}$ were populated with the ${ }^{156} \mathrm{Gd}\left({ }^{35} \mathrm{Cl}, 4 \mathrm{n}\right){ }^{187} \mathrm{Tl}$ reaction at beam energies of 169 and $172 \mathrm{MeV}$. An isotopically enriched target of $0.98 \mathrm{mg} / \mathrm{cm}^{2}{ }^{156} \mathrm{Gd}$ with a backing was used. The $\gamma$ radiation from the target was measured with 12 Comptonsuppressed Ge spectrometers (CSGs) and an inner array of 50 BGO elements. A total of 35 million two- and higher-fold CSG events in prompt coincidence with at least 4 BGO elements was collected.

The proposed level scheme of ${ }^{187} \mathrm{Tl}$ built on the $I^{\pi}=9 / 2^{-}, 16 \mathrm{~s}$ isomer $(E$ $\approx 330 \mathrm{keV}$ [6]) is shown in Fig. II.A.1.1. The parts of this scheme are: (1) a strongly coupled (oblate) band of negative-parity levels on top of the $9 / 2^{-}$isomer; (2) a decoupled (prolate) band of positive-parity levels that becomes yrast at spin 13/2; (3) a decoupled (prolate) band of negative-parity levels.

The major new feature exhibited by our data is the presence of two decoupled rotational bands. The high- $j$ proton orbitals present near the Fermi surface are $h_{9 / 2}$ and $i_{13 / 2}$. They are characterized by a high $K$ value for oblate shapes and a low $K$ 


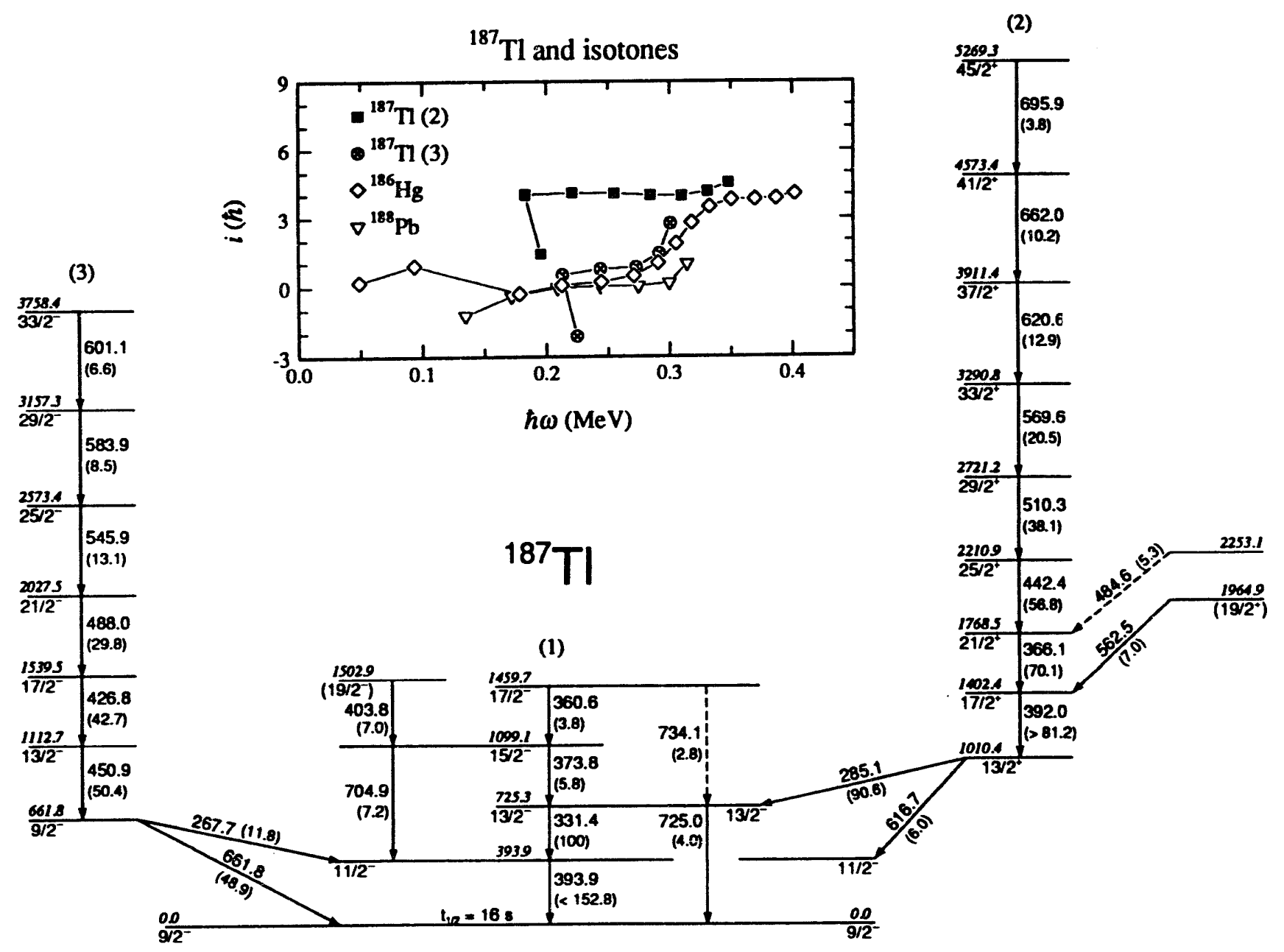

$\bar{A}$

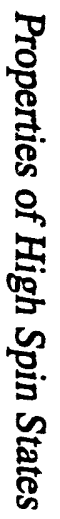

Figure II.A1.1: Partial level scheme of ${ }^{187} \mathrm{Tl}$ obtained in this study. Transitions are labeled by their energy in keV; $\gamma$-ray intensities normalized to 100 for the $331.4 \mathrm{keV}$ transition are given in parentheses. Level energies are given relative to the $9 / 2^{-}$isomeric state. Inset: Aligned angular momentum versus rotational frequency for bands 2 and 3 in ${ }^{187} \mathrm{Tl}$ and prolate bands in ${ }^{186} \mathrm{Hg}[7,8]$ and ${ }^{188} \mathrm{~Pb}[2,3]$. (See text.) 
value for prolate shapes. Even though we do not measure the shape directly, we can infer from the level sequences that band 1 is associated with the commonly observed $\pi h_{9 / 2}$ [505]9/2 configuration in $\mathrm{Tl}$ (slightly oblate), while bands 2 and 3 correspond to the couplings of $i_{13 / 2}$ and $h_{9 / 2}$ protons to the prolate shape seen in the $\mathrm{Hg}$ and $\mathrm{Pb}$ isotones. Band 2 would then be assigned to the [660]1/2 configuration and band 3 to the [541]1/2 or [532]3/2 configuration.

For a quantitative description, bandhead calculations for odd- $A \mathrm{Tl}$ isotopes have been performed using the Nilsson-Strutinsky-BCS method [9]. The values for $\kappa$ and $\mu$ were taken from Ref. [10] and axially symmetric shapes were assumed. The results of these calculations are displayed in Fig. II.A.1.2 as a function of the neutron number, $N$. The upper panel shows the bandhead energies predicted for the lowest-lying prolate and oblate configurations (open symbols) together with the known experimental energies (filled symbols). For prolate deformations, our calculations predict excitation energies for the $i_{13 / 2}$ and $h_{9 / 2}$ bandheads which are close to those observed in this work and for ${ }^{189} \mathrm{Tl}$ estimated in Ref. [4].

The deformation parameters $\epsilon_{2}>0$ corresponding to the calculated bandheads are presented in the middle panel of Fig. II.A.1.2. Predictions for the deformations of the respective $\mathrm{Hg}$ core nuclei (diamonds) are given as well. Focusing on the role of the proton orbitals, two conclusions are important. First, in ${ }^{185} \mathrm{Tl}$ and ${ }^{187} \mathrm{Tl}$, the $\pi h_{9 / 2}$ and $\pi i_{13 / 2}$ bands have a very similar deformation. Secondly, ${ }^{185} \mathrm{Tl}$ and ${ }^{187} \mathrm{Tl}$ are different from the other $\mathrm{Tl}$ isotopes in that the deformations of the prolate $\pi h_{9 / 2}$ configurations are significantly larger $\left(\epsilon_{2} \approx 0.23\right.$ for $N=104$ and 106 , compared to 0.14 or less for the other cases). Based on an inspection of the Nilsson diagram, the prolate $h_{9 / 2}$ configuration is ascribed to the [532]3/2 orbital for $N=104$ and 106, but to the [541]1/2 orbital for the other isotopes. Thus, at the larger deformation $\epsilon_{2}$ $\approx 0.23$ the $h_{9 / 2}$ excitation involves three protons. Both low- $K$ components of the $h_{9 / 2}$ orbital mix at $\hbar \omega>0$, as inferred from CSM calculations.

In the lower panel of Fig. II.A.1.2, the occupation probablities $V^{2}$ calculated for the $\left(\pi h_{9 / 2}\right)^{2} K=1 / 2$ and $3 / 2$ and $\left(\pi i_{13 / 2}\right)^{2} K=1 / 2$ quasiparticle configurations at two different deformations $\epsilon_{2}$ are histogrammed. The left bin corresponds to the $\epsilon_{2}$ value suitable for the $h_{9 / 2}$ configuration, the right bin to the corresponding $\epsilon_{2}$ value for the $i_{13 / 2}$ orbital. Large sum values $\sum V^{2} \approx 1$ for these three relevant proton orbitals at $102 \leq N \leq 108$ result from the enhanced population of all three downsloping orbitals at $\epsilon_{2}>0.2$. While the largest contribution to $\sum V^{2}$ is due to the $\left(\pi h_{9 / 2}\right)^{2} K=1 / 2$ configuration, it is too simplistic to attribute the prolate minimum solely to the $\left(\pi h_{9 / 2}\right)^{2}$ configuration. Note that at $N=104$ and 106 the occupation probabilities for the prolate $h_{9 / 2}$ and $i_{13 / 2}$ bandheads are very similar. This corresponds to the fact that their deformations in ${ }^{185,187} \mathrm{Tl}$ and the deformations 


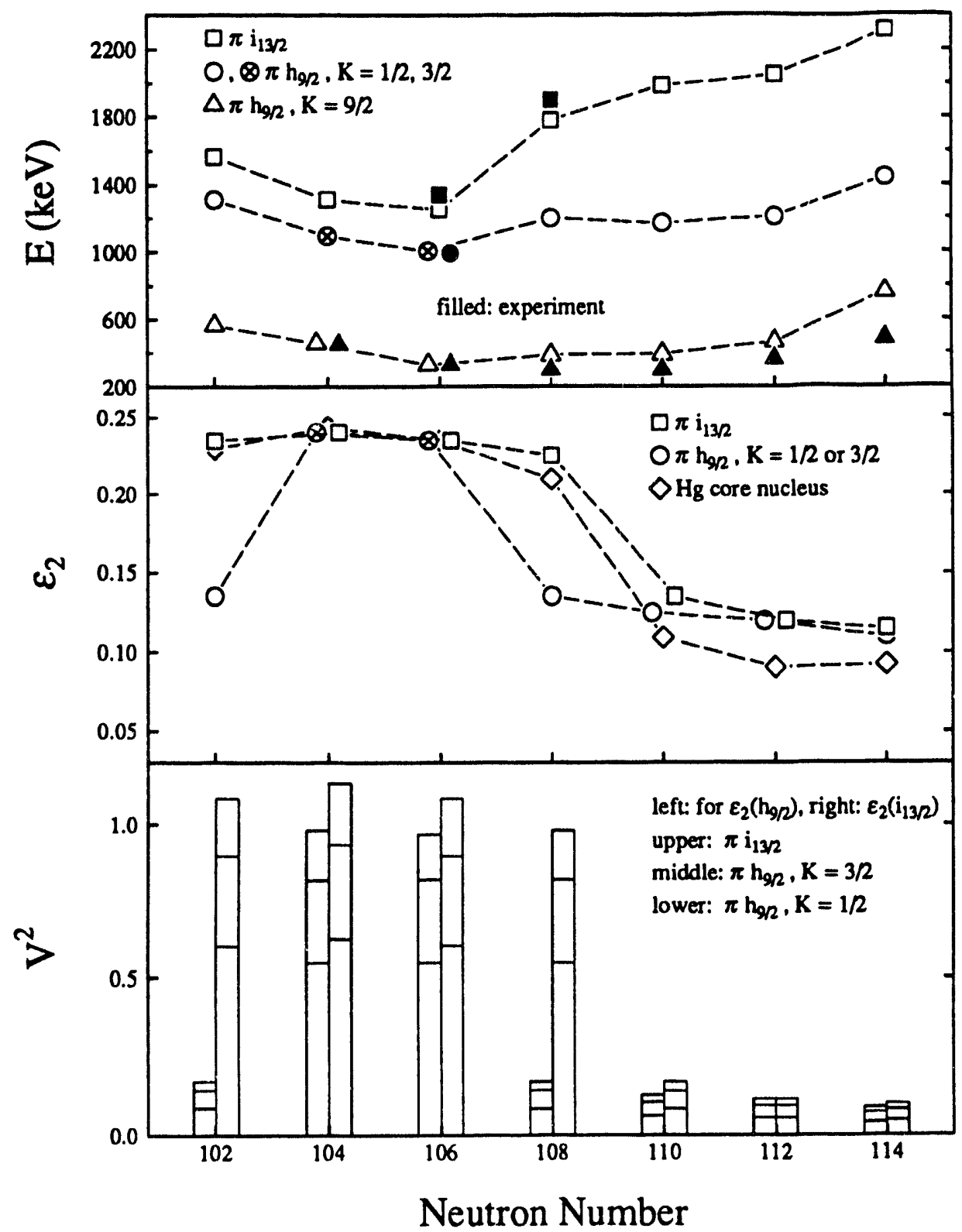

Figure II.A.1.2: Upper panel: Calculated bandhead energies for $\pi i_{13 / 2} K=1 / 2$ (squares), $\pi h_{9 / 2} K=1 / 2$ or $3 / 2$ (circles) and $\pi h_{9 / 2} K=9 / 2$ configurations (triangles) in odd- $A$ Tl isotopes compared with experimental energies from this work, Ref. [4] and Ref. [11]. Middle panel: Calculated quadrupole deformations for the same cases and for $\mathrm{Hg}$ isotones. Lower panel: Occupation probabilities for low- $K$ proton configurations in the respective $\mathrm{Hg}$ cores at two different $\epsilon_{2}$ values (left and right bin). Note that all calculations correspond to zero rotational frequency. 
in the respective $\mathrm{Hg}$ cores are very similar (middle panel). The lack of evidence for a prolate $h_{9 / 2}$ configuration in ${ }^{189} \mathrm{Tl}$ can be explained, alternatively to Ref. [4]. Since at $N=108$ the deformation of a expected $h_{9 / 2}$ band is small compared to that for the $i_{13 / 2}$ structure, the latter will be populated much more strongly at high spins.

The high-spin features of the prolate bands in ${ }^{187} \mathrm{Tl}$ are examined through the alignment plot shown in the inset of Fig. II.A.1.1. The plot includes also the alignments of the prolate bands in ${ }^{186} \mathrm{Hg}[7,8]$ (built on the $0_{2}^{+}$state) and in ${ }^{188} \mathrm{~Pb}$ $[2,3]$. The choice of reference parameters $\left(\mathcal{J}_{0}=26 \hbar^{2} \mathrm{MeV}^{-1}\right.$ and $\mathcal{J}_{1}=200 \hbar^{4}$ $\mathrm{MeV}^{-3}$ ) is arbitrary and done here to give a constant alignment for the $\pi i_{13 / 2}$ band in ${ }^{187} \mathrm{Tl}$. Note that this parameter set works approximately for both prolate bands in ${ }^{187} \mathrm{Tl}$ and also for the prolate bands in the two isotones. This observation supports our calculations, i.e. the bandhead deformations for the two structures in ${ }^{187} \mathrm{Tl}$ and for ${ }^{186} \mathrm{Hg}$ are indeed very similar. This similarity is also suggested by the onset of an upbending (due to a $\left(v i_{13 / 2}\right)^{2}$ alignment) in each band in the same frequency range ( $\hbar \omega=0.30-0.35 \mathrm{MeV}$ ), which should occur if the deformations are similar.

\section{II.A.2. Search for "Normal" and Superdeformed Bands in ${ }^{189} \mathrm{Tl}$.}

W. Reviol, L.L. Riedinger, C.R. Bingham, W.F. Mueller, B.E. Zimmerman [UTK]; C.-H. Yu [Rochester]; I-Y. Lee, A.O. Macchiavelli [LBL].

We have just completed a measurement on ${ }^{189} \mathrm{Tl}$ using GAMMASPHERE in the early implementation phase at the Lawrence Berkeley Laboratory. The purpose of this measurement was to study both large deformed and superdeformed rotational bands in ${ }^{189} \mathrm{Tl}$.

Prolate bands in light Tl nuclei resulting from the coupling of $\pi i_{13 / 2}$ and $\pi h_{9 / 2}$ excitations to the prolate shape in $\mathrm{Hg}$ have attracted considerable interest. Most recently, Lane et al. [12] reported on a study of the yrast $\pi i_{13 / 2}$ bands in ${ }^{185}, 187 \mathrm{Tl}$. In our measurement on ${ }^{187} \mathrm{Tl}$ [5] (see section II.A.1.) we observed not only the low lying slightly oblate structure but also co-existing prolate $\pi i_{13 / 2}$ and $\pi h_{9 / 2}$ bands. Remarkably, both prolate bands in ${ }^{187} \mathrm{Tl}$ seem to have a very similar quadrupole deformation $\beta_{2} \approx 0.25\left(\epsilon_{2} \approx 0.23\right)$. The presence of both configurations is in some contrast to the result from a recent measurement on ${ }^{189} \mathrm{Tl}$ by Porquet et al. [4], where the prolate $\pi i_{13 / 2}$ band was seen, but not the corresponding prolate $\pi h_{9 / 2}$ structure. The latter result lead the authors of Ref. [4] to the conclusion that the prolate minimum is primarily composed of $\left(\pi h_{9 / 2}\right)^{2}$, and thus that the crossing of the $\pi h_{9 / 2}$ structures in ${ }^{189} \mathrm{Tl}$ is blocked. According to the bandhead calculations for odd$A \mathrm{Tl}$ isotopes presented in the previous contribution to this report and in Ref. [5], the deformation for a prolate $\pi h_{9 / 2}$ band in ${ }^{189} \mathrm{Tl}$ is predicted to be significantly smaller 
$\beta_{2} \approx 0.15\left(\epsilon_{2} \approx 0.14\right)$ than that of the prolate $i_{13 / 2}$ band $\left(\beta_{2} \approx 0.25\right)$. The predicted energy of this band, however, is about $800 \mathrm{keV}$ above the oblate $h_{9 / 2}$ bandhead in ${ }^{189} \mathrm{Tl}$, and not far above what we see in ${ }^{187} \mathrm{Tl}$. This structure in ${ }^{189} \mathrm{Tl}$ would be more weakly populated than that in ${ }^{187} \mathrm{Tl}$, since it should have a smaller moment of inertia (due to the smaller deformation), and thus the higher spin levels should lie at higher energy relative to the well deformed $i_{13 / 2}$ band, but it should be observable. So, the the results on ${ }^{189} \mathrm{Tl}[4]$ and the calculations are compatible, as long as a more sensitive measurement does find the expected prolate $h_{9 / 2}$ band. It is important to note that the conclusion about the nature of this lowest-lying prolate minimum in $\mathrm{Hg}$ nuclei depends on the eventual observation of a prolate $\pi h_{9 / 2}$ band in ${ }^{189} \mathrm{Tl}$.

The other purpose for measuring ${ }^{189} \mathrm{Tl}$ was a search for superdeformed bands at $N=108$, both to try to answer some of the key questions on the superdeformed structure and also to probe the edges of this region of superdeformation.

Satula et al. predict [13] that the largest variation in $\beta_{2}$ of the superdeformed band occurs at the "edge" of the region, around ${ }^{189} \mathrm{Tl}$ and ${ }^{190} \mathrm{~Pb}$. That is, while $\beta_{2}$ is rather consistent for superdeformed bands in ${ }^{191} \mathrm{Tl},{ }^{193} \mathrm{Tl}$, and ${ }^{195} \mathrm{Tl}\left(\beta_{2} \approx 0.47\right)$, the value for ${ }^{189} \mathrm{Tl}$ drops by around $10 \%$ [13]. Such a small but significant change in $\beta_{2}$ would be difficult to detect in a quadrupole moment measurement, but perhaps could be verified through understanding signature splitting in the $\pi i_{13 / 2}[642] 5 / 2$ band, which is proposed to be the superdeformed structure in odd- $A$ Tl nuclei. As indicated in the contribution to this report on signature splitting for normal deformed bands (section II.A.8), the $v i_{13 / 2}$ signature splitting is very dependent on the deformation of the nucleus in that configuration. Indeed, we feel that signature splitting can also be used as an indication of the quadrupole deformation. Cranking calculations of the superdeformed $\pi i_{13 / 2}$ configuration indicate that the signature splitting at a rotational frequency of $0.2 \mathrm{MeV}$ should double from about 20 to 40 $\mathrm{keV}$ for a decrease in $\beta_{2}$ by $10 \%$, i.e. from a value of 0.47 in ${ }^{191} \mathrm{Tl}$ to a value $10 \%$ less in ${ }^{189} \mathrm{Tl}$. Such a fingerprint of the changing deformation between different superdeformed Tl nuclei should be observable. The exact degree of the energy splitting between the two signatures of a superdeformed band is not yet known for $\mathrm{Tl}$, since the bandhead energies are generally unknown and connecting transitions between two signatures were not seen. However, we could compare an "energy shift" between two cascades of superdeformed transitions, as described in references [14] and [15]. Fig. II.A.2.1 shows this energy shift $\Delta E$ between the two signatures of the superdeformed $\pi i_{13 / 2}$ band in ${ }^{191} \mathrm{Tl},{ }^{193} \mathrm{Tl}$, and ${ }^{195} \mathrm{Tl}$, which is related to the signature splitting. The $\Delta E$ values are remarkably consistent for these odd- $A \mathrm{Tl}$ nuclei reflecting a very similar deformation. The question is how this energy shift is precisely related to signature splitting, and thus how would an expected doubling 


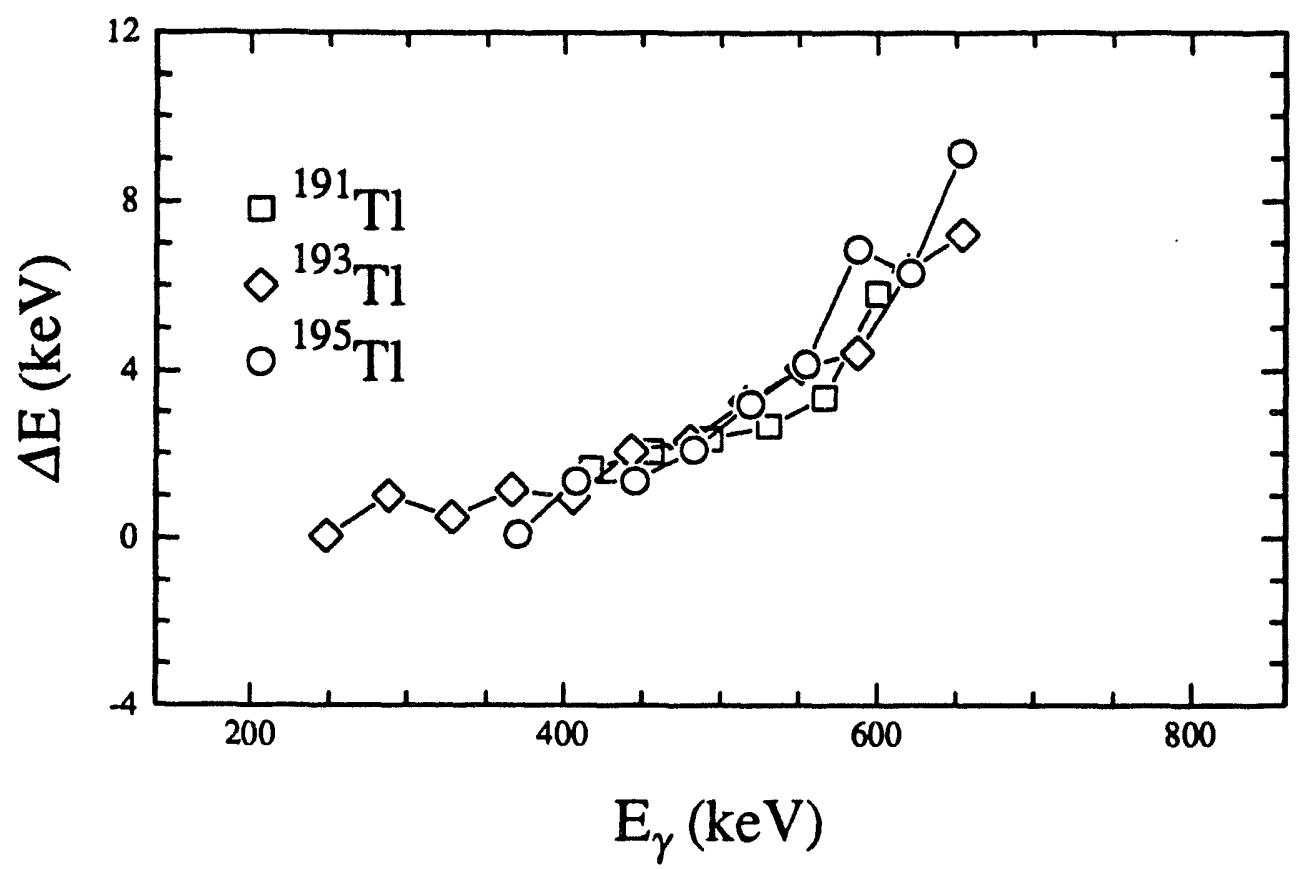

Figure II.A.2.1: Energy shifts, $\Delta E$, measured for superdeformed bands in ${ }^{191} \mathrm{Tl}$ [17], ${ }^{193} \mathrm{Tl}[18]$, and ${ }^{195} \mathrm{Tl}[19]$.

of the signature splitting be detected in terms of this energy shift. To estimate that relationship, we have compared the two quantities for the normal deformed $v i_{13 / 2}$ band ([624]9/2) in two $N=107$ isotopes, ${ }^{181} \mathrm{~W}$ and ${ }^{185} \mathrm{Pt}$ [16]. The former has a known signature splitting of only $3 \mathrm{keV}$, the latter has a value of $36 \mathrm{keV}$. The corresponding energy shifts between the two signatures in these two cases are 6 and $17 \mathrm{keV}$, respectively, at a similar rotational frequency. So, it is clear that an increased signature splitting does result in a larger energy shift. If a superdeformed [642]5/2 band is observable in ${ }^{189} \mathrm{Tl}$, we hope to see an increase in the shift between the two sets of $\Delta I=2$ sequences, compared to the constant trend for the heavier isotopes.

Excited states in ${ }^{189} \mathrm{Tl}$ were populated in the ${ }^{156} \mathrm{Gd}\left({ }^{37} \mathrm{Cl}, 4 \mathrm{n}\right)$ reaction using a ${ }^{37} \mathrm{Cl}$ beam of $171 \mathrm{MeV}$ from the 88 "Cyclotron. The choice of the bombarding energy was based on the experience from populating superdeformed bands in ${ }^{191,193} \mathrm{Tl}$ and ${ }^{190} \mathrm{Hg}$ via $(\mathrm{HI}, 4 \mathrm{n})$ reactions. The final ${ }^{189} \mathrm{Tl}$ nucleus was formed under conditions of $\mathrm{E}_{x} \approx 25 \mathrm{MeV}$ and $l_{\max } \approx 41 \hbar$ and appeared as the dominant evaporation residue. The setup of GAMMASPHERE comprised a total of 34 detectors (early implementation phase). These were 29 detectors grouped at forward and backward angles with respect to the beam axis, the rest (including one "double-D" detector) at $90^{\circ}$. A total 
of 280 million triple and higher fold coincidences were collected during about $70 \mathrm{~h}$ running time. The data analysis is in progress.

\section{II.A.3. Multiple Band Structures in ${ }^{191} \mathrm{Tl}$}

J.M. Lewis, L.L. Riedinger, S. Pilotte, W. Reviol and C.-H. Yu** [UTK], C. Baktash, J.D. Garrett, N.R. Johnson, I.Y. Lee, F.K. McGowan [ORNL].

The data taken for the study of superdeformation in ${ }^{191} \mathrm{Tl}$ [17] have also enabled an investigation of the normal deformed states of this nucleus in great detail. The data are from three experiments. The first experiment, ${ }^{159} \mathrm{~Tb}\left({ }^{36} \mathrm{~S}, 4 \mathrm{n}\right)$ at a bombarding energy of $165 \mathrm{MeV}$, was performed at the Holifield Facility at ORNL using the Spin Spectrometer and 19 Compton-suppressed Ge detectors (CSGs). The second, ${ }^{169} \mathrm{Tm}\left({ }^{26} \mathrm{Mg}, 4 \mathrm{n}\right)$ at $128 \mathrm{MeV}$, was also performed at Holifield using the compact ball with 20 CSGs. Analyzing data from these two experiments has allowed us to extend the known level scheme of high-spin states [20] by 70 transitions, 23 of which were added since last reporting [15]. The third experiment was performed at the ATLAS facility at ANL with the same $\left({ }^{36} \mathrm{~S}, 4 \mathrm{n}\right)$ reaction as in the first experiment. Data from this experiment were used mainly to determine DCO ratios.

The level scheme is shown in Fig. I.A.3.1. It consists essentially of three structures, two of which have positive parity ( 2 and 3 ), the other of which has negative parity. A complete analysis of intensities and DCO ratios has been performed and the results confirm the placement of transitions and spin assignments of levels except in cases noted by dotted lines or parentheses, respectively. The negative-parity $E 2$ sequences based on the $9 / 2^{-}$state [21] (and labeled 1) exhibit a rotationallike behavior and are viewed as being strongly coupled bands. The positive-parity branch, built on the $13 / 2^{+}$state [22] appears to be more complex (structures 2 and 3). First, the level spacings do not show the smooth increase with spin that is usually associated with rotational-like behavior. Secondly, the crossover dipole transitions in this branch compete strongly with the $E 2$ transitions. Thirdly, these two structures seem to be of a different character.

The negative-parity $E 2$ sequences have been interpreted as nearly oblate $\left(\beta_{2}\right.$ $\left.\approx 0.15, \gamma \approx-57^{\circ}\right) \pi h_{9 / 2}$ bands in which a $\nu i_{13 / 2}$ band crossing occurs at $\hbar \omega_{c} \approx 0.22$ $\mathrm{MeV}$ [23]. This agrees with comparable level structures in neighboring isotopes, e.g. ${ }^{193} \mathrm{Tl}$ [24], as well as in $\mathrm{Hg}$-core nuclei [25]. The more interesting point, however, is the interpretation of the level structures built on the long known $13 / 2^{+}$state, in particular band 2. The different characters of both structures could be explained by assuming a nearly spherical shape for structure 3 (similar to the discussion of the positive-parity branch of ${ }^{193} \mathrm{Tl}$ in Ref. [24]), but a deformed shape for band 2 . 


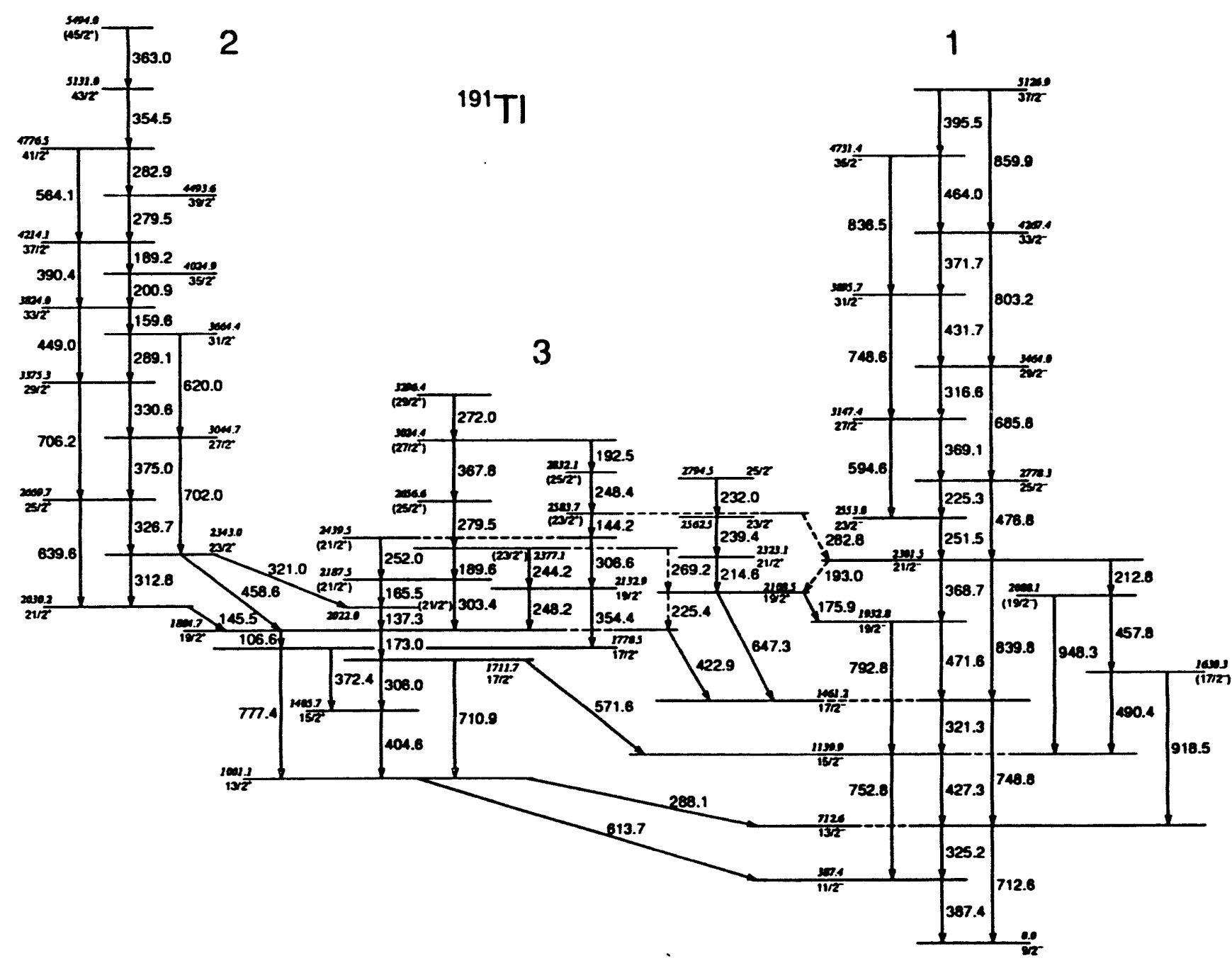

Figure II.A3.1: Level scheme for ${ }^{191} \mathrm{Tl}$. Spin and parity assignments given in parentheses are tentative. 


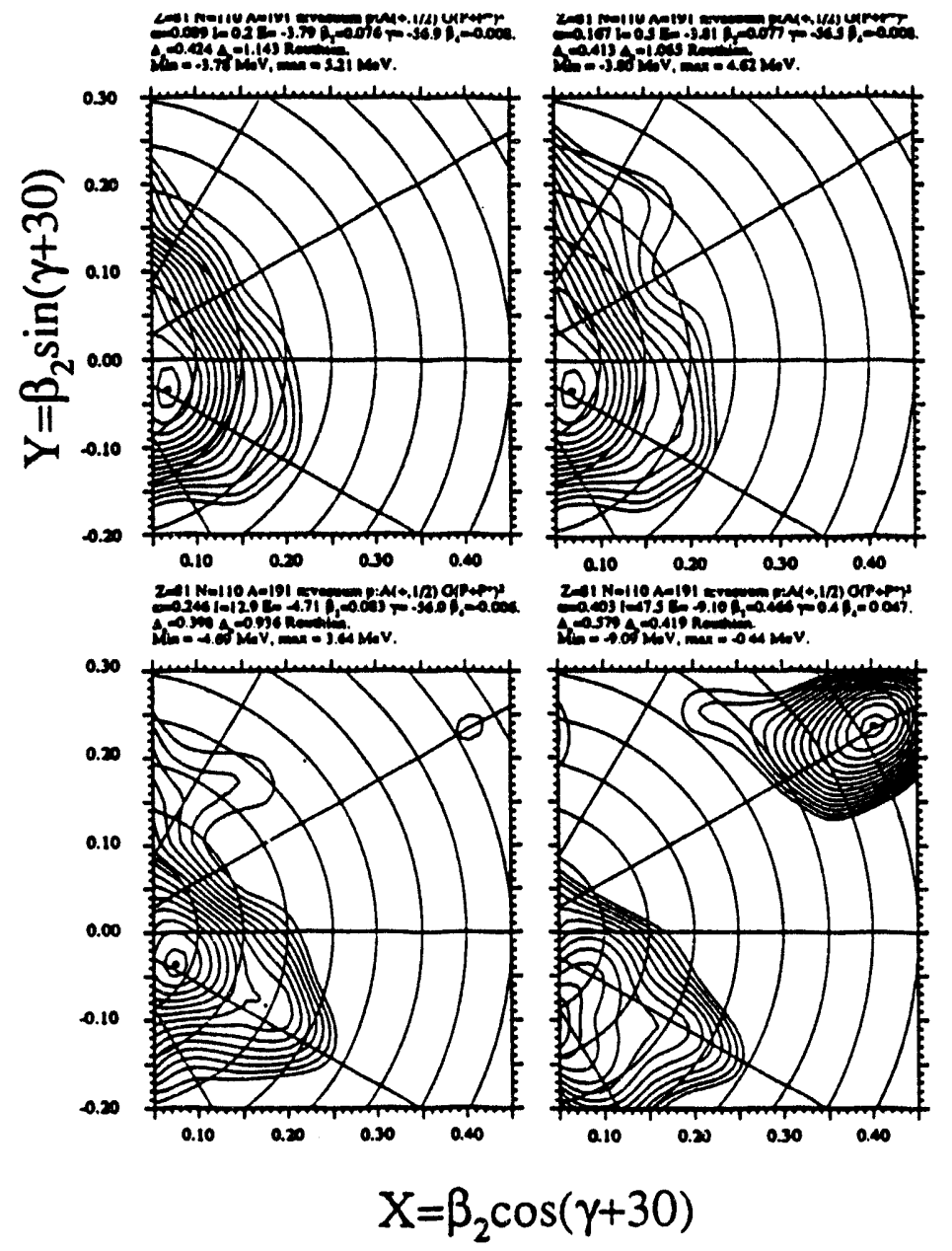

Figure II.A.3.2: Sample TRS plots for positive-parity configurations of ${ }^{191} \mathrm{Tl}$. The frequency range shown is $0.089 \mathrm{MeV} \leq \hbar \omega \leq 0.403 \mathrm{MeV}$.

These alternatives are reflected by Total Routhian Surface (TRS) calculations [26] for positive-parity states in ${ }^{191} \mathrm{Tl}$. Some results from these calculations are shown in Fig. II.A.3.2. At low rotational frequency ( $\hbar \omega<0.24 \mathrm{MeV}$ ) we see only a near-spherical minimum. When the rotational frequency increases, a new deformed minimum tends to develop $\left(\beta_{2} \approx 0.19, \gamma \approx-55^{\circ}\right)$.

In structure 2 of the level scheme the energy spacings approach a somewhat more rotational-like $E_{\gamma}(I)$ behavior, which could reflect the TRS pattern for $\hbar \omega>$ $0.24 \mathrm{MeV}$, so one possible scenario is that this structure is related to the $\beta_{2}=0.19$ minimum, and is in fact the $\pi i_{13 / 2}$ rotational band. Other evidence that supports this assignment is the existence of a backbend around spin $I=31 / 2^{+}$, which 
in this interpretation would be excepted and is due to the alignment of a pair of $i_{13 / 2}$ neutrons. Also, it would be more likely for this structure to be related to the larger deformed minimum because it begins at and extends to higher spin than does structure 3.

As one goes down a band from higher to lower spin, the nucleus is losing both angular momentum and excitation energy. The $\beta_{2}=0.19$ TRS minimum is not very "stable" but is dependent on the rotational frequency of the nucleus. Therefore, at low angular momentum values, it would no longer be favored over the well defined nearly spherical minimum $\left(\beta_{2} \approx 0.08, \gamma \approx-57^{\circ}\right)$. Structure 3 then, which lies at lower spin than band 2 , is most likely related to this small- $\beta_{2}$ minimum. Since the rotational frequency is small, levels built on this structure would not be expected to exhibit the normal behavior of a rotational band. Also, this minimum lies near the non-collective axis $\left(\gamma=-60^{\circ}\right)$ which, along with the small deformation, would further preclude rotational behavior. Supporting evidence for this theory is the complicated single-particle like structure seen in band 3 . Here there are no dipole sequences with $E 2$ crossovers, rather the transitions are mostly dipole and exhibit no regular behavior. The strongest sequence in the upper part of this band involves the $303.4,189.6,279.5,367.8$ and $272.6 \mathrm{keV}$ transitions. In general, however, the transitions above the $19 / 2^{+}$level become increasingly weak as one moves to higher spin, which is expected since this structure is related to a smaller $\beta_{2}$ and thus is not nearly yrast at higher spins. There are three connecting transitions between bands 2 and $2(145.5,321.0$, and $458.6 \mathrm{keV})$, which form the link between the two modes of this $i_{13 / 2}$ structure.

With the analysis of the data essentially complete, future work on ${ }^{191} \mathrm{Tl}$ will consist of the completion of a master's thesis on this work.

* Permanent address: Dept. of Physics, Univ. of Ottawa, Ottawa, Canacia K1N-6N5.

** Pernanent address: Dept. of Physics, Univ. of Rochester, Rochester, NY 14627.

\section{II.A.4. Level Lifetimes in $\pi i_{13 / 2}$ and $\pi h_{9 / 2}$ Bands of ${ }^{181}$ Ir and ${ }^{187} \mathrm{Au}$.}

J.C. Walpe, U. Garg, S. Naguleswaran, Q. Ren [Notre Dame]; W. Reviol, L.L. Riedinger, X.Z. Wang, J.-Y. Zhang [UTK]; R.V.F. Janssens, I. Ahmad, I.G. Bearden, M.P. Carpenter, R.G. Henry, T.L. Khoo, T. Lauritsen [ANL].

In recent years, $\gamma$-spectroscopic studies on series of $\mathrm{Ta}$, Re and Ir nuclei have shown that the low- $K h_{9 / 2}$ and $i_{13 / 2}$ proton intruder orbitals in these nuclei behave very differently at high-spin. This difference is most clearly visible in the experimental alignments $i(\hbar \omega)$ obtained for both configurations, see e.g. the compilation in section II.A.5 of Ref. [15]. From comparisons with neighboring even- $A$ isotones, a $\left(\nu i_{13 / 2}\right)^{2}$ alignment at $\hbar \omega \sim 0.3 \mathrm{MeV}$ is expected. While the 


\begin{tabular}{||l|l|l|l|l||}
\hline $\mathrm{I}_{i}-\mathrm{I}_{f}$ & $\begin{array}{l}E_{\gamma} \\
(\mathrm{keV})\end{array}$ & $\begin{array}{l}\tau \\
(\mathrm{ps})\end{array}$ & $\begin{array}{l}B(E 2) \\
\left(\mathrm{e}^{2} \mathrm{~b}^{2}\right)\end{array}$ & $\begin{array}{l}\mathrm{Q}_{t} \\
(\mathrm{eb})\end{array}$ \\
\hline $13 / 2^{-}-9 / 2^{-}$ & 187.8 & $210(18)$ & $1.16(10)$ & $6.03(52)$ \\
$17 / 2^{-}-13 / 2^{-}$ & 335.6 & $14.8(1.2)$ & $1.20(10)$ & $6.03(49)$ \\
$21 / 2^{-}-17 / 2^{-}$ & 455.8 & $3.1(0.3)$ & $1.30(13)$ & $6.19(60)$ \\
$25 / 2^{-}-21 / 2^{-}$ & 545.9 & $1.3(0.2)$ & $1.31(20)$ & $6.18(95)$ \\
$29 / 2^{-}-25 / 2^{-}$ & 604.9 & $0.8(0.1)$ & $1.16(16)$ & $5.77(72)$ \\
$33 / 2^{-}-29 / 2^{-}$ & 621.6 & $2.6(0.3)$ & & \\
& & & & \\
$212^{+}-17 / 2^{+}$ & 295.0 & $11.8(0.9)$ & $2.81(21)$ & $9.11(69)$ \\
$25 / 2^{+}-21 / 2^{+}$ & 385.0 & $3.4(0.3)$ & $2.70(24)$ & $8.86(78)$ \\
$29 / 2^{+}-25 / 2^{+}$ & 433.3 & $4.5(0.5)$ & & \\
\hline
\end{tabular}

Table II.A.4.1: Preliminary results for ${ }^{181}$ Ir. The lifetimes for the $33 / 2^{-}$and $29 / 2^{+}$ levels could not be separated from the feeding times. The values given are therefore upper limits.

$\pi h_{9 / 2}$ bands upbend as expected, there is no indication for rotational alignment in the $\pi i_{13 / 2}$ bands. Instead, a gradual gain of alignment is observed up to the highest frequencies ( $\hbar \omega \sim 0.45 \mathrm{MeV}$ ). A reasonable interpretation for this "anomalous" behavior of the $\pi i_{13 / 2}$ bands is that the quadrupole deformation is larger than that for the $\pi h_{9 / 2}$ bands. For the latter, deformation parameters have been deduced from the quadrupole moments measured for low-lying $\pi h_{9 / 2}$ states in some Ir and Os nuclei (e.g. $\left.{ }^{181} \mathrm{Ir}[27]\right)$. In order to test this idea of a deformation induced delay of a rotational alignment, we have performed lifetime measurements on ${ }^{181} \mathrm{Ir}$ and ${ }^{187} \mathrm{Au}$ using the recoil distance method.

The ${ }^{154} \mathrm{Sm}\left({ }^{31} \mathrm{P}, 4 \mathrm{n}\right){ }^{181} \mathrm{Ir}$ and ${ }^{154} \mathrm{Sm}\left({ }^{37} \mathrm{Cl}, 4 \mathrm{n}\right){ }^{187} \mathrm{Au}$ reactions were used at beam energies of $140 \mathrm{MeV}$ and $160 \mathrm{MeV}$, respectively. The experiments were performed at the Argonne Tandem Linac Accelerator System using the "plunger" device of the University of Notre Dame in conjunction with the BGO $\gamma$-ray facility at ANL. This facility comprises a BGO ball with $K=50$ elements surrounded by 12 Comptonsuppressed Ge counters (CSGs). An acceptable event consisted of at least one CSG firing in prompt coincidence with $K \geq 4 \mathrm{BGO}$ elements. In both experiments, the target was isotopically enriched ${ }^{154} \mathrm{Sm}$ of $1.1 \mathrm{mg} / \mathrm{cm}^{2}$ thickness evaporated onto a $1.5 \mathrm{mg} / \mathrm{cm}^{2}$ thick Au layer. The stopper was a $22.1 \mathrm{mg} / \mathrm{cm}^{2}$ thick Au foil. Runs of approximately two hours were taken at twenty target-to-stopper distances ranging from 10 to $10000 \mu \mathrm{m}$. 


\begin{tabular}{||l|l|l|l|l||}
\hline $\mathrm{I}_{i}-\mathrm{I}_{f}$ & $\begin{array}{l}E_{\gamma} \\
(\mathrm{keV})\end{array}$ & $\begin{array}{l}\tau \\
(\mathrm{ps})\end{array}$ & $\begin{array}{l}B(E 2) \\
\left(\mathrm{e}^{2} \mathrm{~b}^{2}\right)\end{array}$ & $\begin{array}{l}\mathrm{Q}_{1} \\
(\mathrm{eb})\end{array}$ \\
\hline $13 / 2^{-}-9 / 2^{-}$ & 233.4 & $123(15)$ & $0.77(09)$ & $4.93(60)$ \\
$17 / 2^{-}-13 / 2^{-}$ & 334.7 & $13.7(1.1)$ & $1.31(11)$ & $6.30(51)$ \\
$21 / 2^{-}-17 / 2^{-}$ & 413.8 & $4.8(0.4)$ & $1.33(11)$ & $6.27(52)$ \\
$25 / 2^{-}-21 / 2^{-}$ & 491.6 & $2.1(0.2)$ & $1.34(13)$ & $6.25(60)$ \\
$29 / 2^{-}-25 / 2^{-}$ & 566.8 & $3.8(0.3)$ & & \\
& & & & \\
$212^{+}-17 / 2^{+}$ & 316.4 & $10.4(0.9)$ & $2.27(20)$ & $8.19(71)$ \\
\hline
\end{tabular}

Table II.A.4.2: Preliminary results for ${ }^{187} \mathrm{Au}$. The lifetime for the $29 / 2^{-}$state is an upper limit.

Software gates on higher folds $K$ were used in the analysis, in order to enhance the transitions between high-spin states in ${ }^{181} \mathrm{Ir}$ and ${ }^{187} \mathrm{Au}$, respectively. In ${ }^{181} \mathrm{Ir}$, level lifetimes for the two bands of interest were determined up to $I^{\pi}=33 / 2^{-}$and $25 / 2^{+}$, respectively. Preliminary results are given in Table II.A.4.1. Note that these results are in agreement with a previous measurement for the four lowest yrast trasitions in ${ }^{181}$ Ir [27]. Similarly, in ${ }^{187}$ Au lifetime information was obtained up to $I^{\pi}=29 / 2$ - and $21 / 2^{+}$, respectively, see Table II.A.4.2. In each case, the $\pi i_{13 / 2}$ band is associated with a quadrupole moment $Q_{t}$ approximately $20 \%$ larger than that of the $\pi h_{9 / 2}$ band.

In order to present these results in a more transparent way, the measured quadrupole moments have been transformed into deformation parameters $\epsilon_{2}$ under the assumption of axial symmetric shapes $\left(\gamma=0^{\circ}\right)$ without a hexadecapole degree of freedom $\left(\epsilon_{4}=0\right)$. These $\epsilon_{2}$ values are shown in in Fig. II.A.4.1. It is obvious that in both nuclei the $\pi i_{13 / 2}$ bands are more deformed than the $\pi h_{9 / 2}$ bands. However, it appears that this difference in deformation decreases when going from ${ }^{181} \mathrm{Ir}$ to ${ }^{187} \mathrm{Au}$.

It is interesting to compare this trend with the corresponding quadrupole deformations predicted by TRS calculations (see Fig. II.A.5.2 of Ref. [15]). These calculations show the same trend, i.e. indicate that for light Ir nuclei the $\pi i_{13 / 2}-$ $\pi h_{9 / 2}$ deformation difference is a larger than for Au nuclei with similar neutron numbers. To proceed further in this comparison, we might first concentrate on the isotopic chain of the Ir nuclei. We note that e.g. at $N=102$ the predicted deformation for the $\pi i_{13 / 2}$ band is $27 \%$ larger than that of the $\pi h_{9 / 2}$ band, and this difference decreases with increasing neutron number (18\% at $N=104)$ indicating the influence of the neutron system. However, this influence cannot account solely 


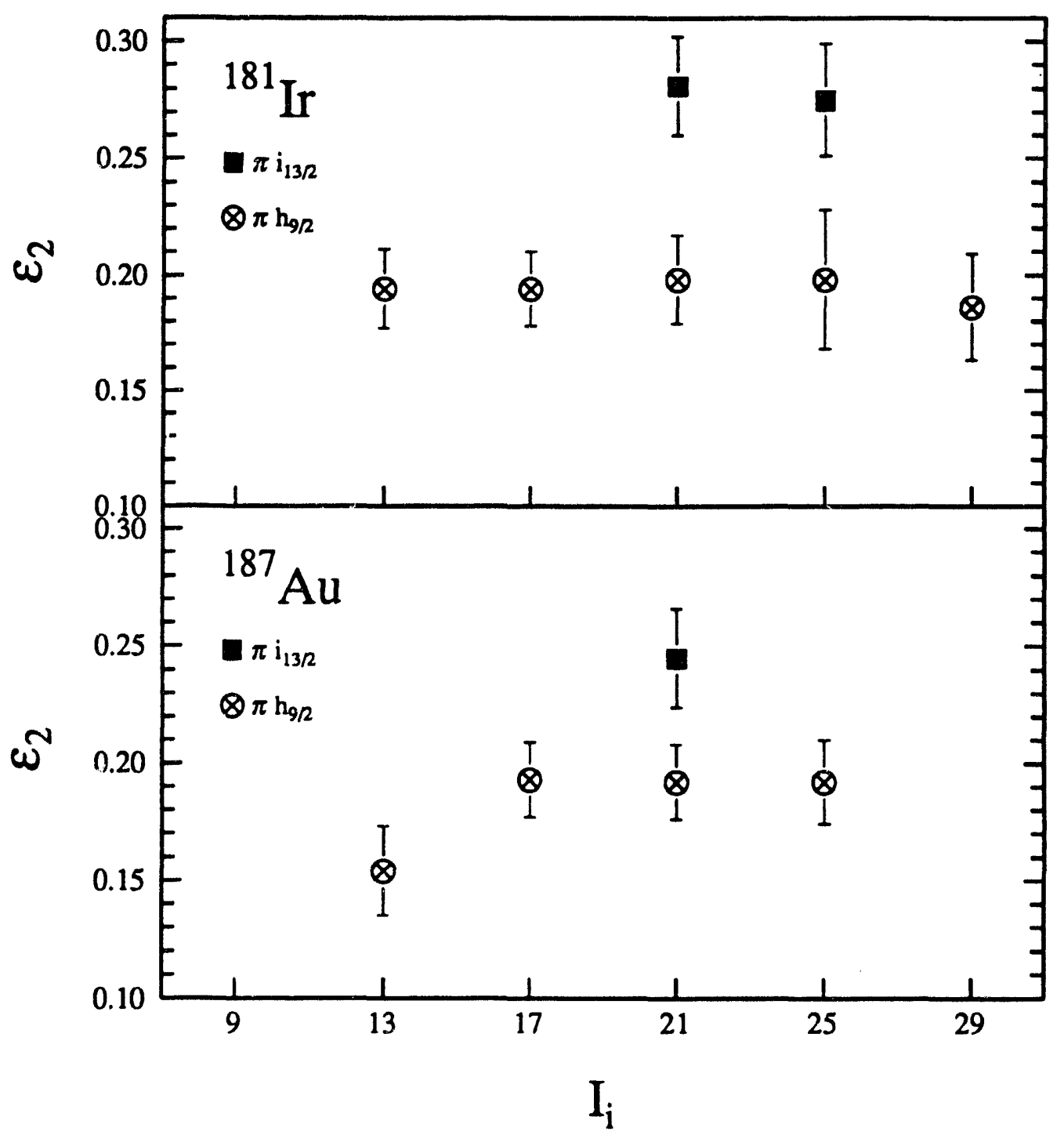

Figure II.A.4.1: Deformation parameters $\epsilon_{2}$ versus spin of the given state for $\pi i_{13 / 2}$ and $\pi h_{9 / 2}$ bands in ${ }^{181} \mathrm{Ir}$ (top) and ${ }^{187} \mathrm{Au}$ (bottom), assuming $\gamma=0^{\circ}$ and $\epsilon_{4}=0$. 
for the predicted difference according to the calculations. For example, at $N=102$, the deformation difference predicted for $\mathrm{Au}(19 \%)$ is significantly smaller than the one for Ir (quoted above). This, clearly shows the effect on the deformation by the proton system when approaching the $Z=82$ closed shell.

\section{II.A.5. New Results on High Spin States in ${ }^{181} \mathrm{Au}$.}

W.F. Mueller, J.M. Lewis, W. Reviol, L.L. Riedinger, C.-H. Yu* [UTK]; C. Baktash, J.D. Garrett, N.R. Johnson, I.Y. Lee, F.K. McGowan [ORNL].

Last year we reported on intruder orbits in ${ }^{181} \mathrm{Au}$, in which the alignments of the $\pi i_{13 / 2}$ and $\pi h_{9 / 2}$ bands of ${ }^{181} \mathrm{Au}$ were presented. In that article, we noted an experiment performed in December 1991 that established bands in ${ }^{181} \mathrm{Au}$. Data from this experiment expands on the older ${ }^{181} \mathrm{Au}$ data presented in our 1988 Progress Report [28]. The December 1991 experiment was performed at the Holifield Heavy Ion Research Facility at Oak Ridge National Laboratory, using the Spin Spectrometer in conjunction with 19 Compton-suppressed Ge detectors. The reaction ${ }^{150} \mathrm{Sm}\left({ }^{35} \mathrm{Cl}, x \mathrm{n}\right)$ at $168 \mathrm{MeV}$ was used, where ${ }^{181} \mathrm{Au}$ is the $4 \mathrm{n}$ channel.

The current level scheme for ${ }^{181} \mathrm{Au}$ is presented in Fig. II.A.5.1. Those transitions that have been added since Ref. [28] are indicated with a dot. At present, the angular correlation analysis has not been completed. However, tentative band assignments can be made from the comparison of the alignments of ${ }^{181} \mathrm{Au}$ bands with bands in ${ }^{183} \mathrm{Au}$ [28] and ${ }^{185} \mathrm{Au}[29]$. The comparison of the alignments of the $\pi i_{13 / 2}$, $\pi h_{9 / 2}$, and $\pi f_{7 / 2}$ bands in ${ }^{183} \mathrm{Au}$ and ${ }^{185} \mathrm{Au}$ with the bands in ${ }^{181} \mathrm{Au}$ are shown in Fig. II.A.5.2. From this comparison, bands $1,2,3$, and 4 can be tentatively labeled as $\pi h_{9 / 2}$ favored signature, $\pi h_{9 / 2}$ unfavored signature, $\pi f_{7 / 2}$, and $\pi i_{13 / 2}$ respectively.

At present, band 5 is thought to possibly be the unfavored signature of the $\pi i_{13 / 2}[660] 1 / 2$ band. No other coincident transitions have been placed yet that would support this hypothesis, and these transitions could be the result of a noncollective effect. Further study is needed, however, before any definitive conclusions can be made.

* Permanent address: Dept. of Physics, Univ. of Rochester, Rochester, NY 14627.

\section{II.A.6. High Spin Studies in ${ }^{164} \mathrm{Tm}$.}

X.Z. Wang, W. Reviol, L.L. Riedinger, J.-Y. Zhang [UTK]; H.J. Jensen, G.B. Hagemann, P.O. Tjøm, R.A. Bark, S. Leoni, T. Lönnroth, H. Schnack-Petersen, T. Shizuma, J. Wrzesinski [NBI].

High spin states of the doubly-odd nucleus ${ }^{164} \mathrm{Tm}$ were populated with the ${ }^{150} \mathrm{Nd}\left({ }^{19} \mathrm{~F}, 5 \mathrm{n}\right)$ reaction at $85 \mathrm{MeV}$. The experiment was performed at the Niels Bohr 


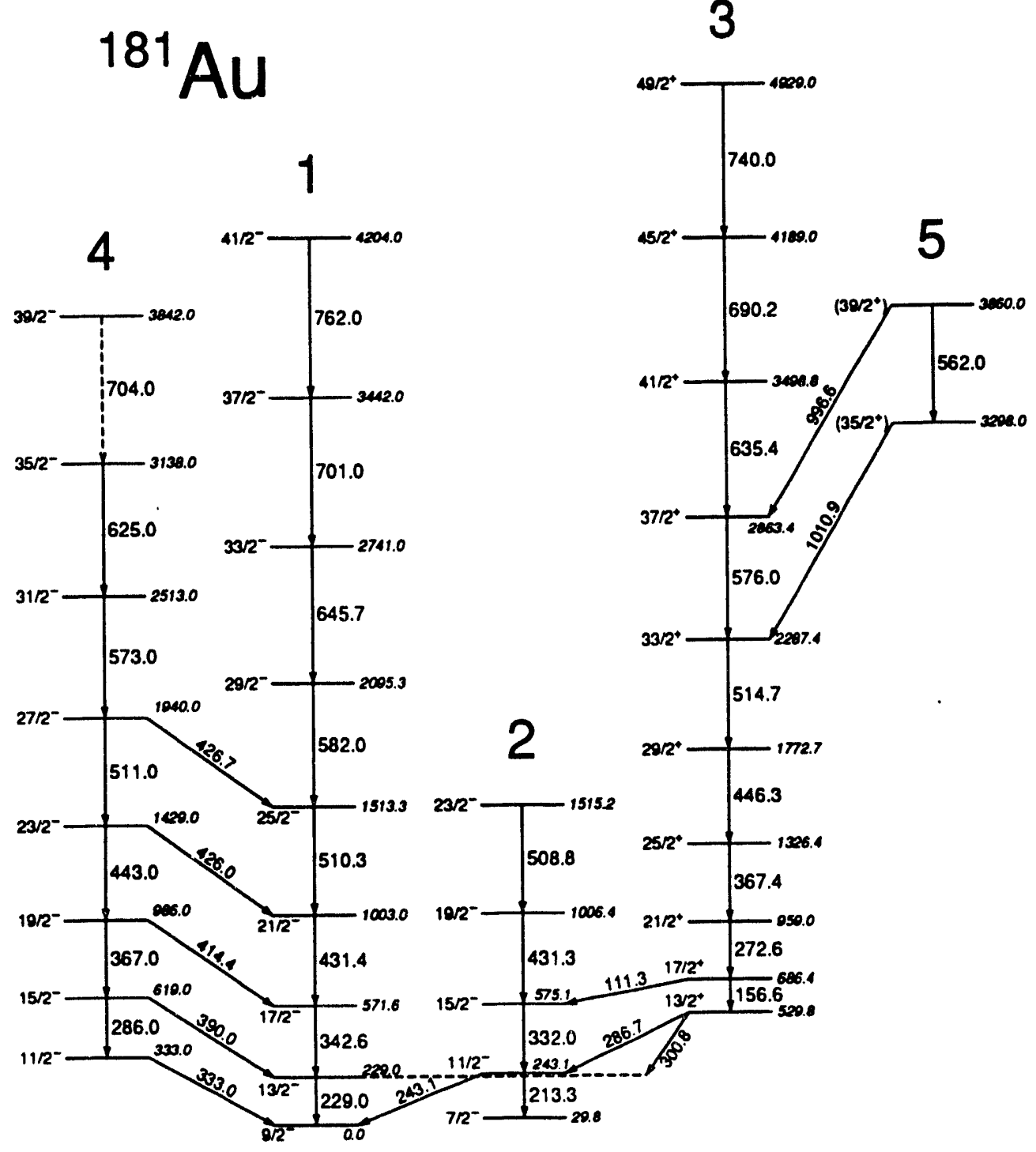

Figure II.A.5.1: Preliminary level scheme of ${ }^{181} \mathrm{Au}$. The lowest level of band 1 is set equal to 0 , as a reference energy. Spin assignments are tentative, based on comparisons with ${ }^{183} \mathrm{Au}$ and ${ }^{185} \mathrm{Au}$ (see text). 


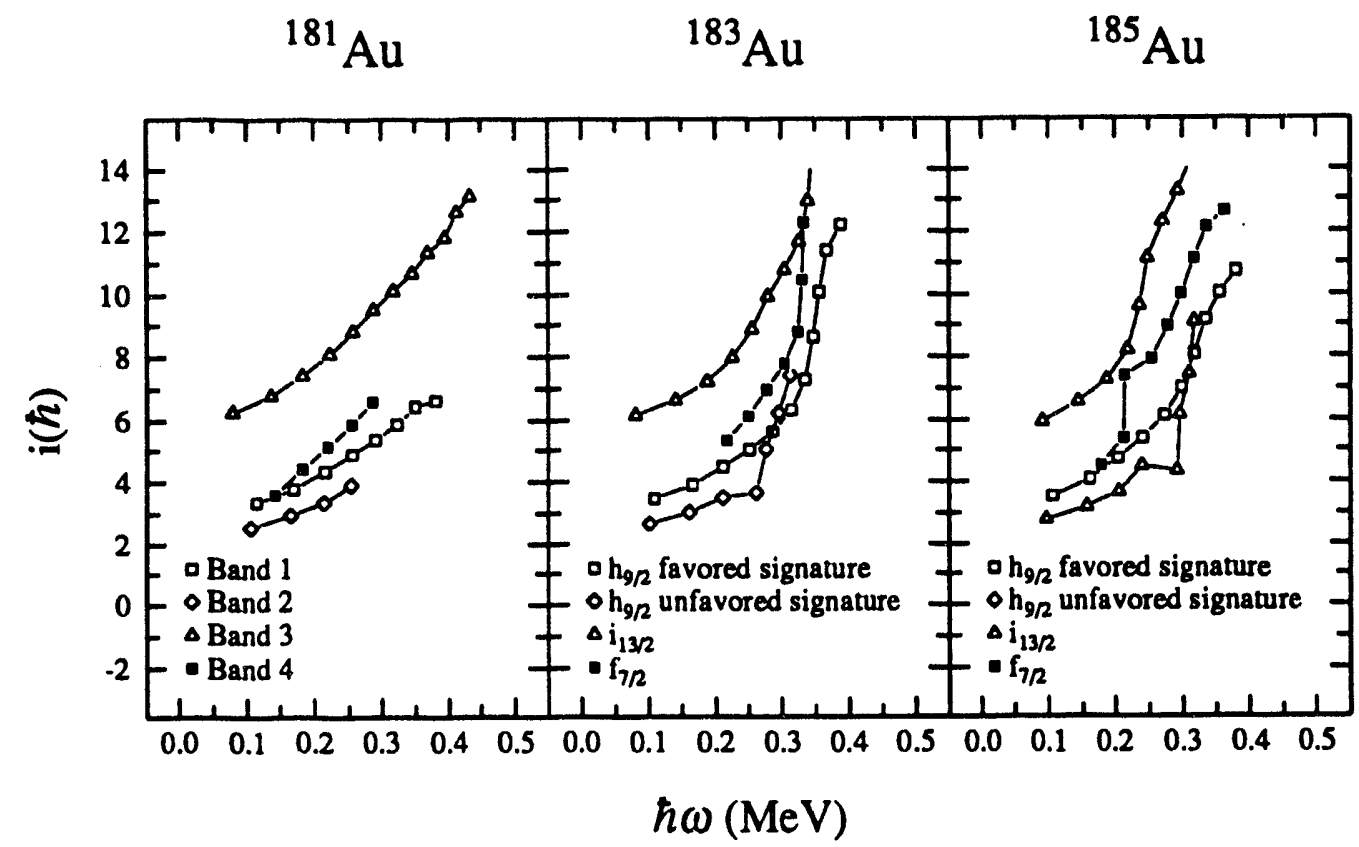

Figure II.A.5.2: Alignment vs. rotational frequency of bands 1 to 4 in ${ }^{181} \mathrm{Au}$ and the $\pi i_{13 / 2}, \pi h_{9 / 2}$, and $\pi f_{7 / 2}$ bands in ${ }^{183} \mathrm{Au}$ and ${ }^{185} \mathrm{Au}$ (references are given in the text).

Institute Tandem Accelerator Laboratory with the Nordball detector system consisting of up to 20 Compton-suppressed Ge detectors (CSGs) situated in four rings and a $\mathrm{BaF}_{2}$ inner ball. Coincident $\gamma$-ray events were collected in two separate measurements, namely with a thin and a backed target. In the thin-target run, the beam was focused on a stack of two self-supporting ${ }^{150} \mathrm{Nd}$ foils, each with a thickness of $0.75 \mathrm{mg} / \mathrm{cm}^{2}$ and an isotopic enrichment of $97.8 \%$. Using all $20 \mathrm{CSGs}$, a total of $1.3 \times 10^{9}$ twofold and higher-fold CSG events in prompt coincidence with at least $1 \mathrm{BaF}_{2}$ element was recorded. The backed target consisted of a $1.35 \mathrm{mg} / \mathrm{cm}^{2}$ thick, isotopically enriched ${ }^{150} \mathrm{Nd}$ foil with an Au backing of $8.3 \mathrm{mg} / \mathrm{cm}^{2}$ thickness. In this case, 18 CSGs plus 2 Ge low energy photon spectrometers (LEPSs) with Compton-suppressors were used and a threshold of 4 was set for the $\mathrm{BaF}_{2}$ elements firing in coincidence with at least 2 CSGs or LEPSs. With this setup, a total of 0.7 $\times 10^{9}$ events was recorded.

Offline, the events from both data sets were sorted into separate $E_{\gamma}-E_{\gamma}$ coincidence matrices after appropriate gain-matching. The sorting was performed under the gating conditions of a high fold $(K)$ and sum energy $(H)$ of the $\mathrm{BaF}_{2}$ elements 


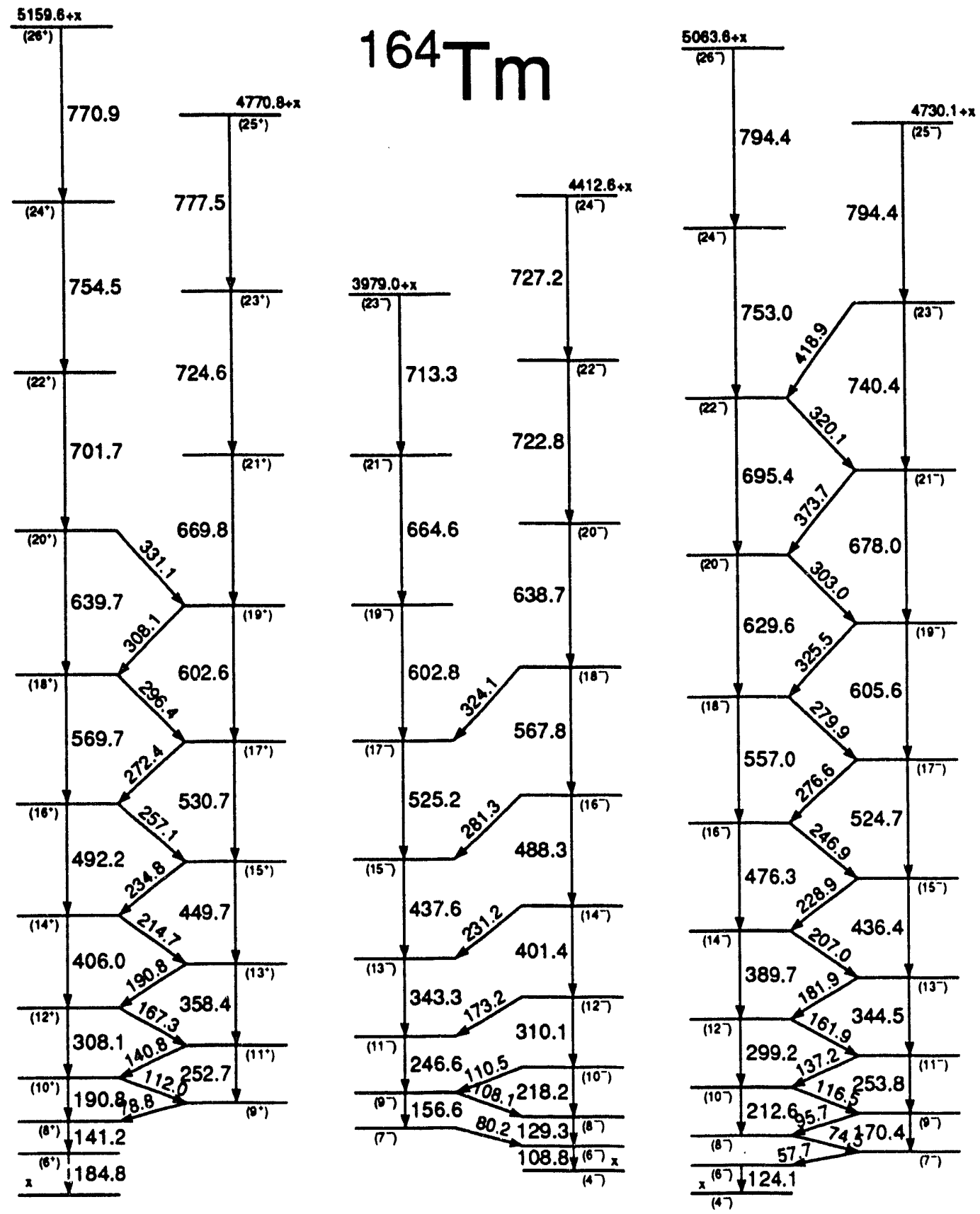

Figure II.A.6.1: Partial level scheme for ${ }^{164} \mathrm{Tm}$. Spin and parity assignments to the bandheads are taken from Ref. [30]. Energy positions of the bandheads are unknown (labeled by $\mathrm{x}$ ). The dashed $184.8 \mathrm{keV}$ transition in the left band is uncertain. The placements of the new transitions in the scheme are based on the coincidence data from both the thin-target and backed-target measurements. 
( $4 \leq K \leq 15$ and $4 \mathrm{MeV} \leq H \leq 20 \mathrm{MeV}$ ) in order to enhance the relative yield of high spin states in ${ }^{164} \mathrm{Tm}$. A subset of angle-sorted matrices was produced with the energies of the events counted in the $37^{\circ}$ and $143^{\circ}$ detector-rings along the $x$-axis and the energies of the events detected under $79^{\circ}$ and $101^{\circ}$ along the y-axis. The DCO (directional correlation from oriented nuclei) ratios obtained from the angle-sorted matrices for the $\gamma$ rays of interest were used to determine their multipolarities.

At the present status of the analysis, the three rotational bands in ${ }^{164} \mathrm{Tm}$ previously known [30] have been extended significantly (up to I $\sim 30 \hbar$ and $\mathrm{E}_{x} \sim 5.2$ $\mathrm{MeV}$ ) and five new band structures of the nucleus have been identified. Fig. II.A.6.1 shows a partial level scheme of ${ }^{164} \mathrm{Tm}$ indicating the three most strongly populated bands. Based on the discussion in Ref. [30], spins and parities are tentatively assigned to the energy levels. It is assumed that the bands shown at the left and the right side are built on $\pi g_{7 / 2}$ (high- $K$ ) $\otimes \nu i_{13 / 2}$ and $\pi h_{11 / 2}$ (high- $K$ ) $\otimes \nu i_{13 / 2}$ configurations, respectively, while the band shown in the middle of the figure is most likely $\pi h_{9 / 2}($ low- $K) \otimes v i_{13 / 2}$ in character. Note that the absolute energy positions of the bandheads are unknown.

\section{II.A.7. Lifetime Measurement for the Superdeformed Band in ${ }^{196} \mathrm{~Pb}$.}

E.F. Moore, H.L. Harrington [North Carolina State]; Y. Liang, R.V.F. Janssens, M.P. Carpenter, I. Ahmad, R.G. Henry, T.L. Khoo, T. Lauritsen [ANL]; I.G. Bearden, P.J. Daly, B. Fornal, Z.W. Grabowski, R.H. Mayer, D. Nissius, M. Sferrazza [Purdue]; M.W. Drigert [IENL]; U. Garg [Notre Dame]; W. Reviol [UTK].

A superdeformed band in ${ }^{196} \mathrm{~Pb}$ was first observed at the LBL 88" Cyclotron populated in ${ }^{176} \mathrm{Yb}\left({ }^{24} \mathrm{Mg}, \mathrm{xn}\right)$ and ${ }^{176} \mathrm{Yb}\left({ }^{26} \mathrm{Mg}, \mathrm{xn}\right)$ reactions [31]. More recently, the nucleus was studied with a similar motivation at ATLAS using the $\left({ }^{30} \mathrm{Si}, 4 \mathrm{n}\right)$ reaction and selfsupporting ${ }^{170} \mathrm{Er}$ targets. In this study, no new superdeformed bands were found in ${ }^{196} \mathrm{~Pb}$, however, it was possible to add new transitions at the top and the bottom of the previously known band, as reported by Moore et al. [32]. Most recently, a lifetime measurement for collective states in ${ }^{196} \mathrm{~Pb}$ with the Doppler Shift Attenuation Method (DSAM) has been performed at ATLAS utilizing the same $\left({ }^{30} \mathrm{Si}, 4 \mathrm{n}\right)$ reaction. Results of this measurement are reported here; they are part of Ref. [32].

For the DSAM measurement, the target consisted of $1.0 \mathrm{mg} / \mathrm{cm}^{2}$ of ${ }^{170} \mathrm{Er}$ evaporated on a $24 \mathrm{mg} / \mathrm{cm}^{2}$ thick $\mathrm{Pb}$ backing. The measurement was carried out at a beam energy of $142 \mathrm{MeV}$ producing the evaporation residues with an initial recoil velocity of $1.5 \%$ speed of light. The $\gamma$ radiation was detected with the BGO $\gamma$-ray facility at ATLAS comprised of a 50-element BGO ball and 12 Compton-suppressed Ge counters (CSGs). A total of $72 \times 10^{6}$ events were collected under the condition 
that at least 2 CSGs and a minimum of 3 BGO elements fired in prompt coincidence. When sorting $\gamma-\gamma$ coincidence matrices for the analysis, a BGO fold $\geq 9$ was required.

Doppler-shifted lineshapes were observed for the transitions in the superdeformed band of ${ }^{196} \mathrm{~Pb}$. From the measured fractions of the full Doppler shift for these transitions, lifetimes were extracted according to the procedure described in Refs. $[33,32]$. These lifetimes correspond to an average quadrupole moment $Q_{t}$ of $18 \pm 3 \mathrm{eb}$.

The measured $Q$, value is in agreement with the quadrupole moments measured for superdeformed bands in neighboring nuclei as well as with several theoretical predictions. A summary of experimental and theoretical $Q_{t}$ values and deformation parameters for superdeformed bands in selected $\mathrm{Hg}$ and $\mathrm{Pb}$ nuclei at $A \sim 190$ is given in Table II.A.7.1. It is found that both experimental and theoretical results are consistent with a quadrupole deformation of $\beta_{2} \sim 0.5$. The data suggest that the deformation reaches a maximum value for $N=112$ in both the $\mathrm{Hg}$ and $\mathrm{Pb}$ superdeformed nuclei. Some of the calculations show the same trend.

\section{II.A.8. Calculated Signature Splitting in W isotopes}

W.F. Mueller, H.J. Jensen, ${ }^{*}$ W. Reviol, L.L. Riedinger, C.-H. Yu, ${ }^{\dagger}$ J.-Y. Zhang [UTK]; W. Nazarewicz ${ }^{\ddagger}$ [JIHIR]; R. Wyss [Manne Siegbahn Institute].

Last year, we demonstrated a method for calculating signature splitting using Total Routhian Surface calculations (TRS) [43], a Wood-Saxon potential based cranked shell model [44], and the Strutinsky Renormalization Procedure. In that report, we included calculations for the $\nu i_{13 / 2}$ bands in isotopes of Dy, Er, and $\mathrm{Yb}$. The agreement of the results of the calculations with the experimentally determined signature splitting was good, with the exception of the lightest $(N=89,91)$ Dy nuclei. The $\nu i_{13 / 2}$ bands in these nuclei, however, are bands with $\Omega \leq \frac{3}{2}$. As a test for $\nu i_{13 / 2}$ bands of higher $\Omega$, calculations were performed for the $\nu i_{13 / 2}$ bands in $\mathrm{W}$ isotopes. The procedure used for calculating the signature splitting involves using deformation parameters predicted from TRS calculations in a cranked shell model program to calculate the signature splitting due to rotation $\left(\Delta E_{r o t}\right)$. These same deformation parameters are then used in a Strutinsky Renormalization procedure to obtain the signature splitting due a possible difference in deformation $\left(\Delta E_{d e f}\right)$.

TRS calculations are divided into groups of nuclei, of which two of these groups contain $\mathrm{W}$ data. One group is centered around ${ }^{168} \mathrm{Yb}$, with limits in $Z$ and $N$ of $64-74$ and $84-104$, respectively. It was from this group that the deformation parameters for the $\mathrm{Dy}, \mathrm{Er}$, and $\mathrm{Yb}$ nuclei were extracted. The other overlaping 


\begin{tabular}{|c|c|c|c|c|c|c|c|c|c|c|c|}
\hline \multirow{2}{*}{$\begin{array}{c}\text { Nucleus } \\
A\end{array}$} & \multirow{2}{*}{$\begin{array}{c}\text { Expt. } \\
Q_{0}\end{array}$} & \multicolumn{3}{|c|}{ Chasman } & \multicolumn{3}{|c|}{ Satula } & \multirow{2}{*}{$\begin{array}{c}\text { Meyer } \\
Q_{0}\end{array}$} & \multirow{2}{*}{$\begin{array}{c}\text { Krieger } \\
Q_{0}\end{array}$} & \multicolumn{2}{|c|}{ Bonche } \\
\hline & & $\beta_{2}$ & $\beta_{4}$ & $Q_{0}$ & $\beta_{2}$ & $\beta_{4}$ & $Q_{0}$ & & & $\beta_{2}$ & $Q_{0}$ \\
\hline \multicolumn{12}{|l|}{$\overline{\mathrm{Hg}_{\mathrm{g}}}$} \\
\hline 190 & $18(3)$ & 0.55 & 0.03 & 19.1 & 0.46 & 0.06 & 18.6 & 18.1 & & 0.53 & $\begin{array}{l}17.6^{8} \\
16.0^{h}\end{array}$ \\
\hline 191 & $18(3)^{b}$ & 0.55 & 0.03 & 19.1 & & & & & & & \\
\hline 192 & $\begin{array}{l}20(2)^{c} \\
20(3)^{d}\end{array}$ & 0.55 & 0.03 & i9.2 & 0.48 & 0.07 & 19.9 & 18.2 & 18.0 & 0.54 & $\begin{array}{l}18.5^{8} \\
17.8^{h}\end{array}$ \\
\hline $\begin{array}{l}194 \\
\mathrm{~Pb}\end{array}$ & & 0.50 & 0.03 & 19.3 & 0.47 & 0.06 & 19.3 & 18.5 & & & \\
\hline 192 & & 0.50 & & 17.6 & 0.47 & 0.07 & 19.4 & 18.7 & 17.9 & & \\
\hline 194 & $20(3)$ & 0.55 & & 19.8 & 0.49 & 0.07 & 20.8 & 19.3 & 19.2 & & \\
\hline 196 & $18.3(3)^{f}$ & 0.50 & & 17.9 & 0.48 & 0.06 & 20.5 & 19.2 & 20.0 & & \\
\hline 198 & & 0.55 & & 20.1 & 0.48 & 0.04 & 20.1 & 18.8 & 20.9 & & \\
\hline
\end{tabular}

Table II.A.7.1: Measured and calculated quadrupole moments and deformation parameters in the superdeformed bands of selected $\mathrm{Hg}$ and $\mathrm{Pb}$ isotopes (table taken from Ref. [32]). References for the experimental values: (a) Drigert et al. [34], (b) Moore et al. [35] and Carpenter et al. [36], (c) Moore et al. [33], (d) Lee et al. [14], (e) Willsau et al. [37], and (f) present work. The calculated values are taken from Chasman [38], Satula et al. [13], Meyer et al. [39], Krieger et al. [40], and Bonche et al. [41, 42] (g: obtained from Hartree-Fock + BCS calculations, h: obtained from generator coordinate method calculations). All $Q_{t}$ values are in eb.

group is centered around ${ }^{180} \mathrm{Pt}$ with limits of $Z=72-82$ and $N=94-118$. The Wood-Saxon parameters in each group are optimized for the central nucleus and used for the calculation of the single particle levels of the other nuclei in the group. Because of this, it is expected that there will be differences in the predicted deformation parameters for overlaping nuclei in each group. Fig. II.A.8.1 shows the predicted deformation parameters from both groups for $\mathrm{W}$ nuclei. While the differences in the predicted deformations are not large, the differences are large enough to have a noticeable effect on signature splitting. To be consistent with the previous calculations of $\mathrm{Dy}, \mathrm{Er}$, and $\mathrm{Yb}$, the deformation parameters from the group centered around ${ }^{168} \mathrm{Yb}$ are used for subsequent calculations. For both groups, the predicted $\beta_{2}$ is maximum at $N=101$. After $N=101$ upsloping $v i_{13 / 2}$ bands are becoming more energetically favorable, which results in a decrease in deformation for $N>101$.

Fig. II.A.8.2 shows the calculated and experimental signature splitting at $\hbar \omega$ $=0.2 \mathrm{MeV}$. There are no $v i_{13 / 2}$ signature splitting data for ${ }^{177} \mathrm{~W}(N=103)$, but signature splitting is systematic enough that it is very likely that the value would 


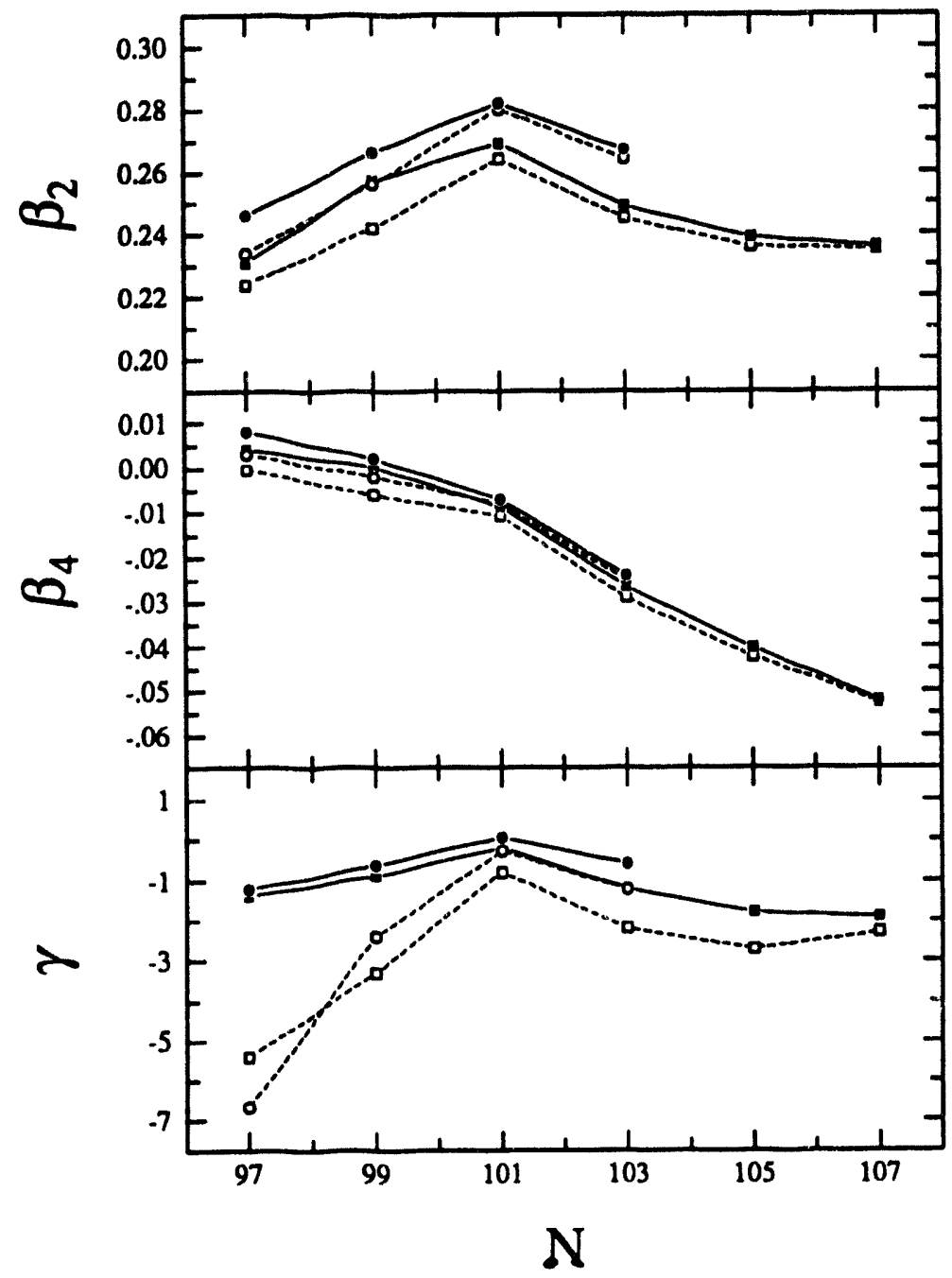

Figure II.A.8.1: Deformation parameters at $\hbar \omega=0.2 \mathrm{MeV}$ for the $v i_{13 / 2}$ bands in odd- $A \mathrm{~W}$ nuclei. These values were extracted from TRS calculations. The open symbols refer to the favored signature $\left(\alpha=\frac{1}{2}\right)$, and the filled symbols to the unfavored signature $\left(\alpha=-\frac{1}{2}\right)$. The values for the $\mathrm{Yb}$ mesh are denoted by circles, the Pt mesh by squares. 


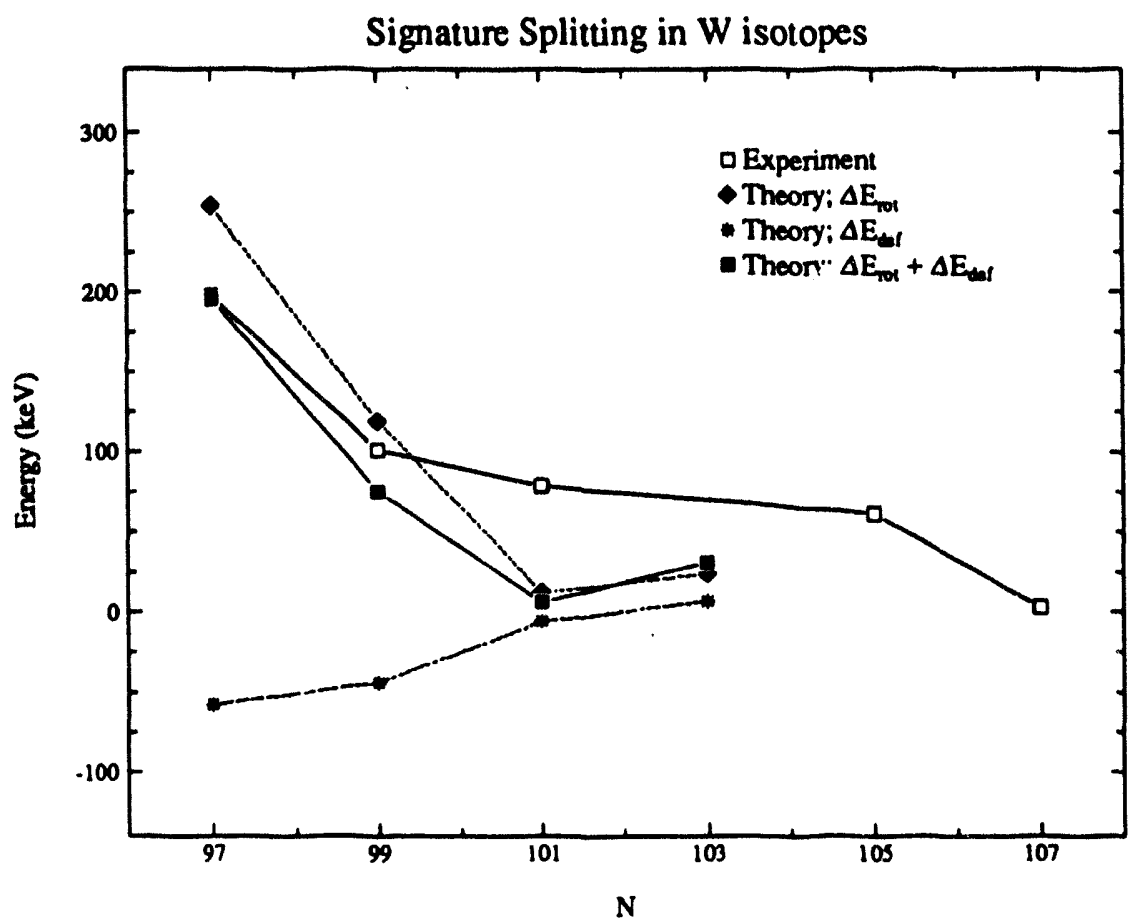

Figure II.A.8.2: Experimental and calculated $v i_{13 / 2}$ signature splitting at $\hbar \omega=0.2$ $\mathrm{MeV}$ for isotopes of W. The experimental data for $A=171 \mathrm{~W}$ isotopes are from Ref. [45], $A=173,175$ are from Ref. [46], $A=179$ from Ref. [47], and $A=181$ from [48].

be $70 \mathrm{keV} \pm 25 \mathrm{keV}$, as seen from the trend of values in the neighboring odd- $N$ $\mathrm{W}$ isotopes. Fig. II.A.8.1 demonstrates that the difference in deformation between the two signatures is greater for the lighter $\mathrm{W}$ isotopes, and therefore the magnitude of $\Delta E_{\text {def }}$ is greater for these isotopes. For $N=97$ and 99 , the agreement of the calculations with experiment is quite good. For $N=101$ and 103, the calculated and experimental results differ by as much as $70 \mathrm{keV}$. It is possible that these deviations are related to the "central nucleus" effect in the TRS mesh. That is, nuclei farther from the central nucleus ${ }^{168} \mathrm{Yb}(Z=70, N=98)$ might be expected to have the most "suspect" deformation parameters. We see that the largest deviations between experiment and calculated signature splitting occur for ${ }^{175} \mathrm{~W}(Z=74, N=101)$ and ${ }^{155} \mathrm{Dy}(Z=66, N=89)$. This could be related to their differences in $N$ from that of the central nucleus.

* Permanent address: Niels Bohr Institute, Denmark. 
† Permanent address: Nuclear Structure Research Laboratory, University of Rochester, 271 East River Road, Rochester, NY 14627.

¥ Permanent address: Institute of Theoretical Physics, Warsaw University, 00-681 Warsaw, Poland; Institute of Physics, Warsaw Institute of Technology, Warsaw, Poland.

\section{II.A.9. Identical Transitions between Superdeformed and Normally Deformed Bands and the Cancellation Picture.}

\section{J.-Y. Zhang, L.L. Riedinger [UTK]; R.F. Casten [BNL].}

According to the criteria proposed by us [49], a systematic search of identical transition sequences between rotational bands with superdeformation (SD) and those with normal deformation (ND) in rare earth and actinide nuclei has been carried out for the first time [50]. Results are shown in Table II.A.9.1. The fact that bands in nuclei so different in deformation/pairing and so separated in mass are identical suggests that probably a mass-deformation/pairing cancellation picture is involved. In this picture the effects of larger mass in a normally deformed nucleus cancel the effect of larger deformation and weaker pairing in the superdeformed nucleus. A standard Nilsson-Strutinsky-BCS type calculation with particle number projection [10] has been done as well. Results show that through a proper selection of the pairing for different bands, the identity of transitional sequences between SD and ND bands can be reached. Other pairs of identical bands in normally deformed or superdeformed nuclei may involve another type of cancellation mechanism $[51,49]$.

\section{II.A.10. Relation of Collective Observables to Nuclear Shapes.}

J.-Y. Zhang [UTK]; R.F. Casten, N.V. Zamfir [BNL].

It is found that $B\left(E 2 ; 2^{+} \rightarrow 0^{+}\right)$values and energy ratios $R\left(4^{+} / 2^{+}\right)$of yrast bands [52] behave differently for transitional nuclei, suggesting that these two observables reflect collectivity in rather different ways. Theoretical deformation values of the ground state for all the even-even nuclei from $Z=30$ up to actinides are obtained through a normal Nilsson-Strutinsky-BCS type potential energy surface (PES) calculation [9]. A standard set of Nilsson parameters $\kappa$ and $\mu$ proposed by Bengtsson and Ragnarsson [53] is used in the calculation. For this set of parameters, $\kappa$ and $\mu$ are assumed to depend on the main oscillator quantum number $N$.

The comparison between experimental $B\left(E 2 ; 2^{+} \rightarrow 0^{+}\right)$and $R\left(4^{+} / 2^{+}\right)$values and theoretical PES results leads to a simple and qualitative distinction between them, namely that the $B\left(E 2 ; 2^{+} \rightarrow 0^{+}\right)$values are primarily sensitive to the location of the minimum in the potential energy surface, while the $R\left(4^{+} / 2^{+}\right)$values seem to reflect the detailed shape and anharmonicities of the potential. 
Table II.A.9.1: $\mathrm{E}_{\gamma}$ comparison between $\mathrm{SD}$ in $\mathrm{Hg}, \mathrm{Pb}$ and $\mathrm{ND}$ in U,Th.

\begin{tabular}{|c|c|c|c|c|c|c|}
\hline \hline $\mathrm{A} 1: \mathrm{A} 2$ & $\mathrm{I}_{f 1}{ }^{1}$ & $\mathrm{E}_{\gamma 1}$ & $\mathrm{I}_{f 2}{ }^{1}$ & $\mathrm{E}_{\gamma 2}$ & $\mathrm{R}\left(\mathrm{J}^{(2)}\right)$ & $\mathrm{R}\left(\mathrm{J}^{(1)}\right)$ \\
\hline${ }^{232} \mathrm{U} \mathrm{yb}^{2}$ & 8 & 218.0 & 10 & 214.6 & & \\
$:$ & 10 & 264.0 & 12 & 257.2 & & \\
${ }^{192} \mathrm{Hg} \mathrm{1b}$ & 12 & 307.0 & 14 & 299.6 & & \\
& 14 & 341.7 & 16 & 340.9 & 0.306 & 0.970 \\
\hline${ }^{236} \mathrm{U} \mathrm{lb}$ & 8 & 212.4 & 10 & 214.6 & & \\
$:$ & 10 & 259.7 & 12 & 257.2 & & \\
${ }^{192} \mathrm{Hg} \mathrm{1b}$ & 12 & 302.7 & 14 & 299.6 & & \\
& 14 & 341.0 & 16 & 340.9 & 0.155 & 0.735 \\
\hline${ }^{238} \mathrm{U} \mathrm{1b}$ & 8 & 210.6 & 10 & 214.6 & & \\
$:$ & 10 & 257.8 & 12 & 257.2 & & \\
${ }^{192} \mathrm{Hg} \mathrm{1b}$ & 12 & 300.6 & 14 & 299.6 & & \\
& 14 & 338.8 & 16 & 340.9 & 0.276 & 0.601 \\
\hline${ }^{232} \mathrm{Th} \mathrm{1b}$ & 10 & 270.1 & 12 & 257.7 & & \\
$:$ & 12 & 310.1 & 14 & 299.9 & & \\
${ }^{192} \mathrm{Hg} \mathrm{1b}$ & 14 & 345.7 & 16 & 340.9 & & \\
& 16 & 375.8 & 18 & 381.1 & 0.538 & 0.813 \\
\hline${ }^{196} \mathrm{~Pb} \mathrm{1b}$ & 14 & 345.7 & 16 & 345.5 & & \\
\hline${ }^{232} \mathrm{Th} 1 \mathrm{~b}$ & 10 & 270.1 & 12 & 262.6 & & \\
$:$ & 12 & 310.1 & 14 & 304.1 & & \\
${ }^{192} \mathrm{~Pb} \mathrm{1b}$ & 14 & 345.7 & 16 & 345.6 & & \\
& 16 & 3758 & 18 & 385.6 & 0.528 & 0.738 \\
\hline${ }^{232} \mathrm{Th} \mathrm{1b}$ & 10 & 270.1 & 12 & 259.0 & & \\
$:$ & 12 & 310.1 & 14 & 302.8 & & \\
& 16 & 375.8 & 18 & 387.3 & 0.721 & 0.835 \\
\hline
\end{tabular}

${ }^{1}$ Subindex 1 and 2 stand for nucleus $\mathrm{A} 1$ and $\mathrm{A} 2$ respectively.

${ }^{2}$ Band labeling, yb for yrast band, $1 \mathrm{~b}$ for $1 \mathrm{~b}$ band. 
[1] J. L. Wood et al., Phys. Rep. 215 (1992), references therein.

[2] J. Heese et al., Phys. Lett. B 302, 390 (1993).

[3] A. M. Baxter et al., Phys. Rev. C, in press (unpublished).

[4] M.-G. Porquet et al., Phys. Rev. C 44, 2445 (1991).

[5] W. Reviol et al., in press, Phys. Rev. C (unpublished).

[6] A. G. Schmidt et al., Phys. Lett. B 66, 133 (1977).

[7] W. C. Ma et al., Phys. Rev. C 47, R5 (1993).

[8] M.-G. Porquet et al., J. Phys. G18, L29 (1992).

[9] S. G. Nilsson et al., Nucl. Phys. A131, 1 (1969).

[10] R. Bengtsson and J.-Y. Zhang, Phys. Lett. B 135, 358 (1984).

[11] K. Heyde et al., Phys. Rep. 102 (1983), references therein.

[12] G. J. Lane et al., submitted to Phys. Lett. B (unpublished).

[13] W. Satula et al., Nucl. Phys. A529, 289 (1991).

[14] L. L. Riedinger, W. F. Mueller, and C.-H. Yu, in International Conference on Nuclear Structure at High Angular Momentum (AECL, Ottawa, Canada, 1992), report 10613 (Vol. 2) p. 339.

[15] Technical report, UTK Nuclear Physics Group, (unpublished), document no. 93-01.

[16] W. F. Mueller et al., in review, Phys. Rev. C (unpublished).

[17] S. Pilotte et al., in International Conference on Nuclear Structure at High Angular Momentum (AECL, Ottawa, Canada, 1992), report 10613 (Vol. 2) p. 2.

[18] P. B. Fernandez et al., Nucl. Phys. A517, 386 (1990).

[19] F. Azaiez et al., Z. Phys. A338, 471 (1991).

[20] L. Goettig et al., Technical report, Nuclear Structure Laboratory, University of Notre Dame (unpublished), page 85.

[21] R. M. Diamond and F. S. Stephens, Nucl. Phys. 45, 632 (1963).

[22] J. O. Newton et al., Nucl. Phys. A236, 225 (1974).

[23] Technical report, UTK Nuclear Physics Group, (unpublished), document no. 92-01.

[24] W. Reviol et al., Nucl. Phys. A548, 331 (1992).

[25] H. Hübel et al., Nucl. Phys. A453, 316 (1986).

126] R. Wyss et al., Nucl. Phys. A511, 324 (1990).

[27] R. Kaczarowski et al., Phys. Rev. C 41, 2069 (1990).

[28] Technical report, UTK Nuclear Physics Group, (unpublished), document no. 88-03.

[29] A. J. Larabee et al., Phys. Lett. B 169, 21 (1986).

[30] S. Drissi et al., Nucl. Phys. A466, 385 (1987).

[31] M. Brinkman et al., Z. Phys A336, 115 (1990).

[32] E. F. Moore et al., Phys. Rev. C 48, 2261 (1993).

[33] E. F. Moore et al., Phys. Rev. Lett. 64, 3127 (1990).

[34] M. W. Drigert et al., Nucl. Phys. A530, 452 (1991).

[35] E. F. Moore et al., Phys. Rev. Lett. 63, 360 (1989).

[36] M. Carpenter et al., Phys. Rev. Lett. B 240, 44 (1990).

[37] P. Willsau et al., Z. Phys. A344, 351 (1993).

[38] R. R. Chasman, Phys. Lett. B 219, 227 (1989).

[39] M. Meyer et al., Phys. Rev. C 45, 233 (1992).

[40] S. Krieger et al., Nucl. Phys. A542, 43 (1992).

[41] P. Bonche et al., Phys. Rev. C 45, 233 (1992).

[42] P. Bonche et al., Nucl. Phys. A519, 509 (1990).

[43] R. Wyss and J. Nyberg, private communication.

[44] W. Nazarewicz et al., Nucl. Phys. A435, 397 (1985).

[45] J. Espino et al., to be published Nucl. Phys. A (unpublished). 
[46] P. M. Walker et al., J. Phys. G4, 1655 (1978).

[47] P. M. Walker et al., Phys. Rev. Lett. 67, 433 (1991).

[48] R. B. Firestone, NDS 43, 289 (1984).

[49] J.-Y. Zhang and L. L. Riedinger, Phys. Rev. Lett. C 69, 3448 (1992).

[50] J.-Y. Zhang, submitted to Phys. Lett. B (unpublished).

[51] 1. Ragnarsson, Nucl. Phys. A520, 67c (1990).

[52] S. Raman et al., At. Data and Nucl. Data Tables 36, 1 (1987).

[53] T. Bengtsson and I. Ragnarsson, Nucl. Phys. A436, 14 (1985). 


\section{II.B. Study of Low-Energy Levess of Nuclei Far from Stability}




\section{II.B.1. Observation of Fine Structure in ${ }^{190} \mathrm{~Pb} \alpha$ Decay}

J. D. Richards, C. R. Bingham, and B. E. Zimmerman [UTK], Y. A. Akovali and K.S. Toth [ORNL], J. A. Becker and E. A. Henry [LLNL], J. Kormicki [Vanderbilt], H. K. Carter and P. F. Mantica, Jr. [UNISOR], E. F. Zganjar and P. Joshi [LSU]

Recent work from Leuven [1,2], has revealed fine structure in decays to excited states of even-even nuclei in the neutron deficient lead region. From their work they have computed hindrance factor (H.F.) values for the purpose of investigating the physics governing these transitions. They interpret the high H.F. values to the difficulty for a nucleus to decay through a "two-step process", whereby in addition to the removal of an $\alpha$ particle two protons are excited to the corresponding "intruder orbital" of the daughter nucleus. In this work we present the first observation of fine structure in ${ }^{190} \mathrm{~Pb} \alpha$-decay and then discuss briefly the systematics of such transitions seen in neighboring nuclei.

$\mathrm{The} \mathrm{Pb}$ isotopes were produced by bombarding natural $\mathrm{W}$ with beams of 170 $\mathrm{Mev}{ }^{16} \mathrm{O}$ from the Holifield Heavy Ion Research Facility 25-MV tandem accelerator. The $A=190$ products were mass-separated utilizing the UNISOR on-line facility and collected onto an automated tape transport system, alowing $\alpha$ particles to be detected at two counting stations. A portion of the $\alpha$ spectra obtained at the first station is provided in Figure II.B.1.1. In addition to the main $\alpha$ peak from the decay of ${ }^{190} \mathrm{~Pb}$ a small, but well-defined peak is also seen. It is observed $398 \pm 2 \mathrm{keV}$ lower than the main $\alpha$-peak. When corrected for the recoil energy, this energy difference yields $407 \pm 2 \mathrm{keV}$, which can be identified as the $2^{+}$level at $405.44 \mathrm{keV}$ in ${ }^{186} \mathrm{Hg}$ [3]. Moreover, the peak has a half-life of $62 \pm 14 \mathrm{~s}$ which agrees with the established half life of ${ }^{190} \mathrm{~Pb}$. Therefore, on the basis of its energy, and half-life, we assign the peak to the decay of the ground state of ${ }^{190} \mathrm{~Pb}$ to the $2_{1}^{+}$state of ${ }^{186} \mathrm{~Pb}$ at $405.44 \mathrm{keV}$. We find the relative intensity of ${ }^{190} \mathrm{~Pb} \alpha$-decay to the excited $2^{+}$state, as compared to transitions to ground state $\alpha$ intensity to be $(8.4 \pm 1.5) \times 10^{-4}$. No transition was seen to the $0_{2}^{+}$state although we estimate an upper limit for possible decays to this level, relative to the ground state, to be less than $1.5 \times 10^{-4}$.

The measured energies of $\alpha$ particles seen from nuclei in this region and corresponding hindrance factors that were obtained from our data are provided in Table II.B.1.1. The experimental value for fine structure $\alpha$-decay from ${ }^{190} \mathrm{~Pb}$ to the $2^{+}$state in ${ }^{186} \mathrm{Hg}$ and the upper limit to decay to the $0_{2}^{+}$is to our knowledge the first in the literature. Also listed in Table II.B.1.1 are the corresponding hindrance factors for fine structure $\alpha$-decay of neighboring nuclei already appearing in the literature [1].

In Figure II.B.1.2 we provide a level scheme of excited states of the daughter nuclei corresponding to some of the $\alpha$ transitions listed in Table II.B.1.1, along with 


\section{Alpha Decay of $190 \mathrm{~Pb}$}

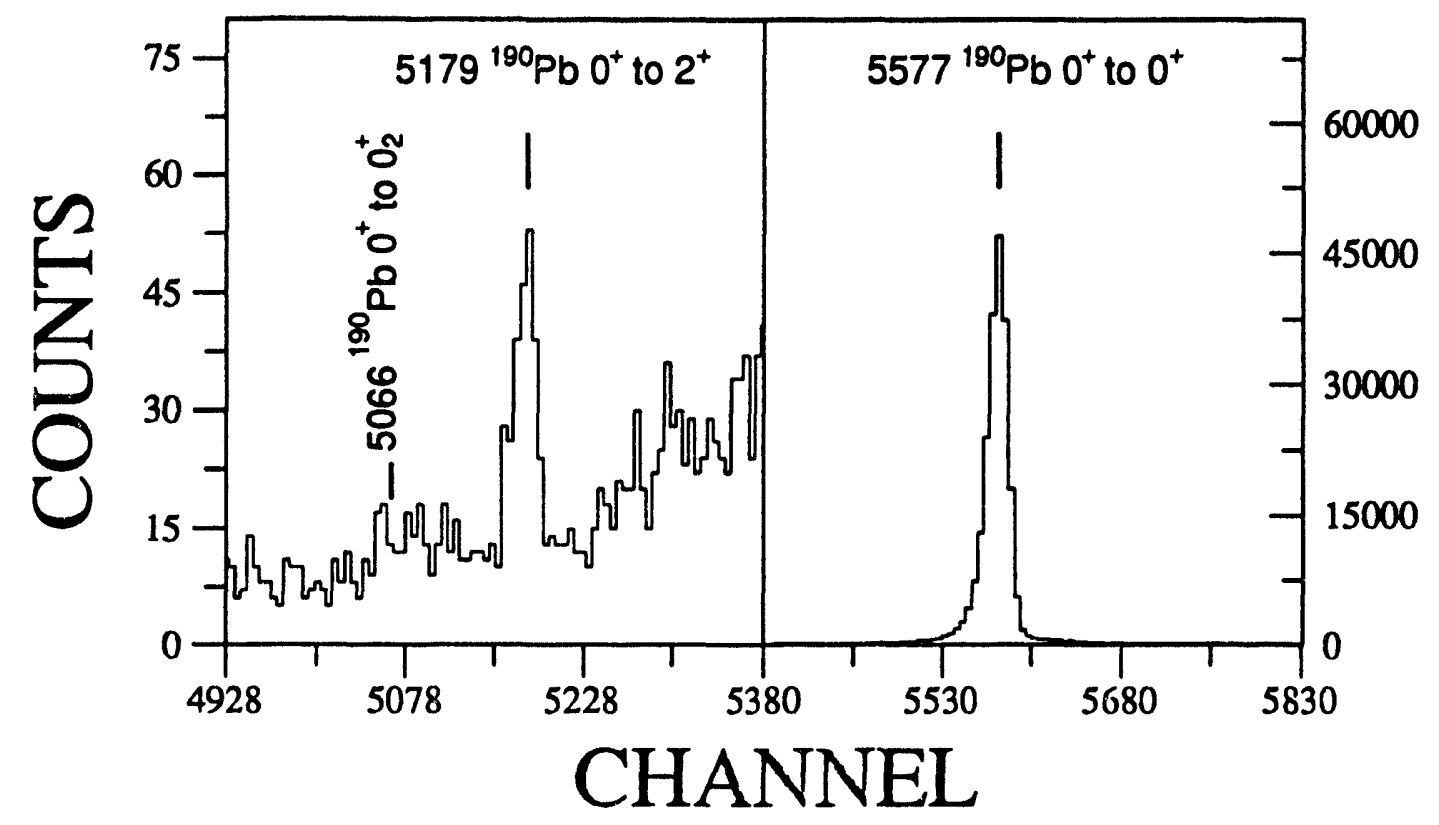

Figure II.B.1.1: Portion of the $\alpha$ spectrum taken at the first counting station for the first four minutes after collection.

H.F. values, one of which includes our new data point. Using the prescription of Rasmussen [4] including the angular momentum induced component of the nuclear barrier, we find the H.F. to be $6 \pm 1$ for $\alpha$ decay in ${ }^{190} \mathrm{~Pb}$, so that it is much less hindered than for the corresponding transition in ${ }^{188} \mathrm{~Pb}$ [1] and ${ }^{186} \mathrm{~Pb}$ [2]. For the case of ${ }^{190} \mathrm{~Pb}$ the considerably lower H.F. value indicates that the $2_{1}^{+}$state in ${ }^{186} \mathrm{Hg}$ is more similar in structure to the ground state than in ${ }^{184,182} \mathrm{Hg}$.

At least for the time being, the lower bound on the transition to the $\mathrm{O}_{2}^{+}$state in ${ }^{186} \mathrm{Hg}$ might be seen as an agreement with the highly hindered case of the corresponding transition to the $\mathrm{O}_{2}^{+}$state in ${ }^{184} \mathrm{Hg}$. If they are ever found to be be the same, then this might help confirm the similar deformation expected for this coexisting band in these nuclei [5].

As shown in Table II.B.1.1 and Figure II.B.1.2 similar rapidly-varying H.F. are observed for fine structure peaks in the decay of ${ }^{180-184} \mathrm{Hg}$. In ${ }^{178} \mathrm{Pt}$ the two lowest states are thought to belong to the strongly deformed band. The next excited level, $\mathrm{O}_{2}^{+}$, is thought to be the weakly deformed bandhead. In ${ }^{176} \mathrm{Pt}$, the two lowest 
Fine Structure in ${ }^{190} \mathrm{~Pb} \alpha$ Decay
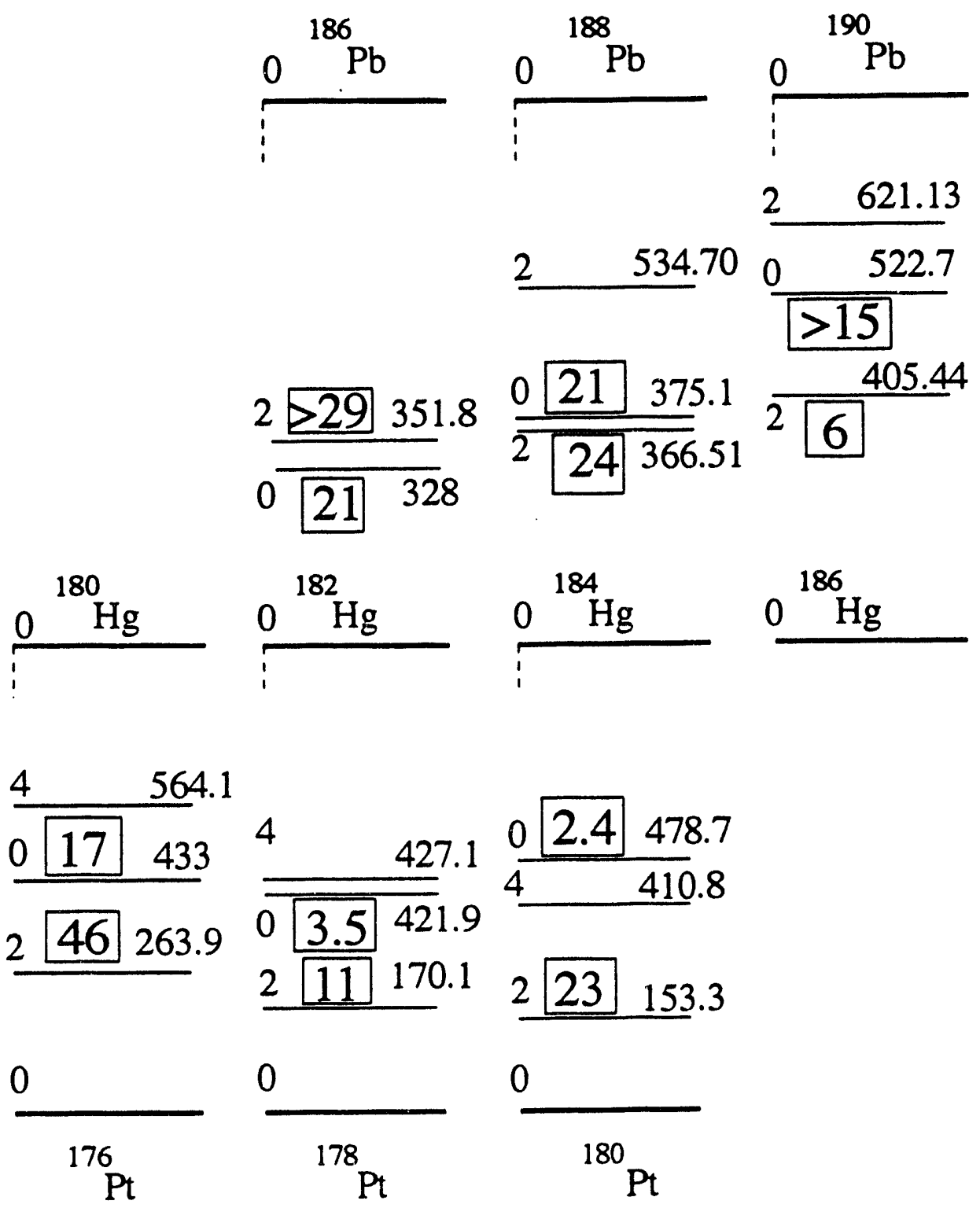

Figure II.B.1.2: Low-lying levels of $\alpha$-decay daughter nuclei of several isotopes of $\mathrm{Pb}$ and $\mathrm{Hg}$. The spins and energies of the levels, and H.F. values (in boxes) are given for levels populated by $\alpha$ decay. 
Table II.B.1.1: Partial $\alpha$-Branches and Hindrance Factors for Fine Structure

\begin{tabular}{||c|c|c|c||}
\hline Parent Nuclide & Transition & $\alpha$ Decay Energy & Hindrance Factor \\
${ }^{190} \mathrm{~Pb}$ & $0_{2}^{+}$ & $5066(5)^{a}$ & $>15^{a}$ \\
& $2^{+}$ & $5179(5)^{a}$ & $6.3(1.0)^{a}$ \\
${ }^{188} \mathrm{~Pb}$ & $0_{2}^{+}$ & $5614(10)^{b}$ & $21(3)^{b}$ \\
& $2^{+}$ & $5633(10)^{b}$ & $24^{c}$ \\
${ }^{186} \mathrm{~Pb}$ & $0_{2}^{+}$ & $6014(10)^{c}$ & $21(4)^{c}$ \\
& $2^{+}$ & & $>29^{c}$ \\
${ }^{184} \mathrm{Hg}$ & $0_{2}^{+}$ & $5067^{c}$ & $3.5(6)^{c}$ \\
& $2^{+}$ & & $23^{c}$ \\
${ }^{182} \mathrm{Hg}$ & $0_{2}^{+}$ & $5446(7)^{b}$ & $2.4(2)^{b}$ \\
& $2^{+}$ & $5689(7)^{b}$ & $11^{c}$ \\
${ }^{180} \mathrm{Hg}$ & $0_{2}^{+}$ & $5689(5)^{b}$ & $17(5)^{b}$ \\
& $2^{+}$ & $5862(5)^{b}$ & $46^{c}$ \\
${ }^{194} \mathrm{Po}$ & $0_{2}^{+}$ & $6194(5)^{b}$ & $1.1(1)^{b}$ \\
${ }^{196} \mathrm{Po}$ & $0_{2}^{+}$ & $5769(5)^{b}$ & $2.5(1)^{b}$ \\
${ }^{198} \mathrm{Po}$ & $0_{2}^{+}$ & $5273(5)^{b}$ & $2.8(1)^{b}$ \\
${ }^{202} \mathrm{Rn}$ & $0_{2}^{+}$ & $5836(5)^{b}$ & $19(6)^{b}$ \\
\hline
\end{tabular}

${ }^{a}$ Values from this work ${ }^{b}$ Values from other work [1] ${ }^{c}$ Values from other work [2]

levels are thought to belong to the weakly deformed band. The next excited state is possibly the $0_{2}^{+}$level of the strongly deformed band [6]. The H.F. values of 3.5 \pm 0.6 and $2.4 \pm 0.2$ for decay of ${ }^{182} \mathrm{Hg}$ and ${ }^{184} \mathrm{Hg}$ to the $0_{2}^{+}$states, belonging to the weakly deformed band in ${ }^{178} \mathrm{Pt}$ and ${ }^{180} \mathrm{Pt}$ indicate unhindered transitions, which one might expect as the ground states of these $\mathrm{Hg}$ nuclei are weakly deformed. With the roles reversed for the strongly and weakly deformed bands of ${ }^{176} \mathrm{Pt}$, it is difficult, however, to interpret the high hindrance to the excited $0_{2}^{+}$state, since the absolute reduced widths for decay to the ground state of the three nuclei, are more similar in magnitude. However the H.F. value for transition to the $2^{+}$level is high in either situation. That the hindrance is much higher for ${ }^{176} \mathrm{Pt}$ could suggest that the $2^{+}$state mixes considerably with the deformed configuration, as its energy seems lowered in relation to the $2^{+}$levels of the weakly deformed band of Pt nuclei [6]. 


\section{II.B.2. Alpha Decay Studies in $\mathrm{Hg}$, Au, Pt Nuclides Using a Double-Sided Silicon Strip Detector}

B. E. Zimmerman, C. R. Bingham, and J. D. Richards [UTK]; K. Toth [ORNL]; H. Penttila, A. Wuosmaa, and C. N. Davids [ANL]; P. Woods [Edinburgh]; L . F. Conticchio [Maryland]; P. F. Mantica, Jr. [ORISE]

Because of the varied distribution of nuclei produced in a heavy-ion reaction, the spectra obtained tend to be very complex and the background high. This is especially true in the production of alpha-emitting nuclei near $A=180$, which makes observation of weak reaction channels and decay of nuclei with small decay branches difficult. A Double-sided Silicon Strip Detector (DSSD) [7] similar to that developed at Daresbury has been implemented with the Fragment Mass Analyzer (FMA) at Argonne National Laboratory for such studies.

The DSSD consists of a set of $48 \times 48$ orthogonal strips etched onto a $60 \mu \mathrm{m}$-thick $\mathrm{Si}$ wafer, effectively producing over 2300 pixels. This provides for the gathering of time, position, and energy data for each event whether it is an implantation or decay. In addition, mass information is obtained by correlating each pixel event with the position in a Parallel-Plate Avalanche Counter (PPAC) of the last implanted recoil in that same pixel. The full power of the detector, however, comes in its ability to correlate alpha decay daughters and their parents.

In order to search for the alpha-decaying nucleus ${ }^{180} \mathrm{~Pb}$, the reaction of 216 $\mathrm{MeV}{ }^{40} \mathrm{Ca}$ on isotopically-enriched targets of ${ }^{144} \mathrm{Sm}$ was used and the recoils with $A=178,179,180$ were focussed on the PPAC. The recoils were subsequently deposited into the DSSD. Time, energy, and position data were gathered in the DSSD and mass data were collected in the PPAC. Despite the fact that no $\mathrm{Tl}$ or $\mathrm{Pb}$ was produced in the reaction (although PACE predicts production of $\sim 10-50 \mu \mathrm{b}$ ), the experiment proved useful in learning how to use the detector and how to get the most of the data gathered.

Figure II.B.2.1 shows an ungated singles spectrum, showing only decay events for $A=178,179,180$. Figure II.B.2.2 shows the same spectrum with a gate requiring that the events be due to alpha decay from $A=179$ recoils. This greatly simplifies the spectrum.

By not only requiring the correct mass of the recoil, but also noting which decays follow each other in the same pixel, parent-daughter relationships can be determined. Since the count rate in each pixel was kept low (several seconds between recoils), most alphas could be correctly correlated with their recoil parent. If granddaughter activity were to be produced (it was not in this experiment), the granddaughters would also be correlated with their parents (the daughter of the implanted recoil). Figure II.B.2.3 shows a 2-dimensional histogram of alpha-alpha 


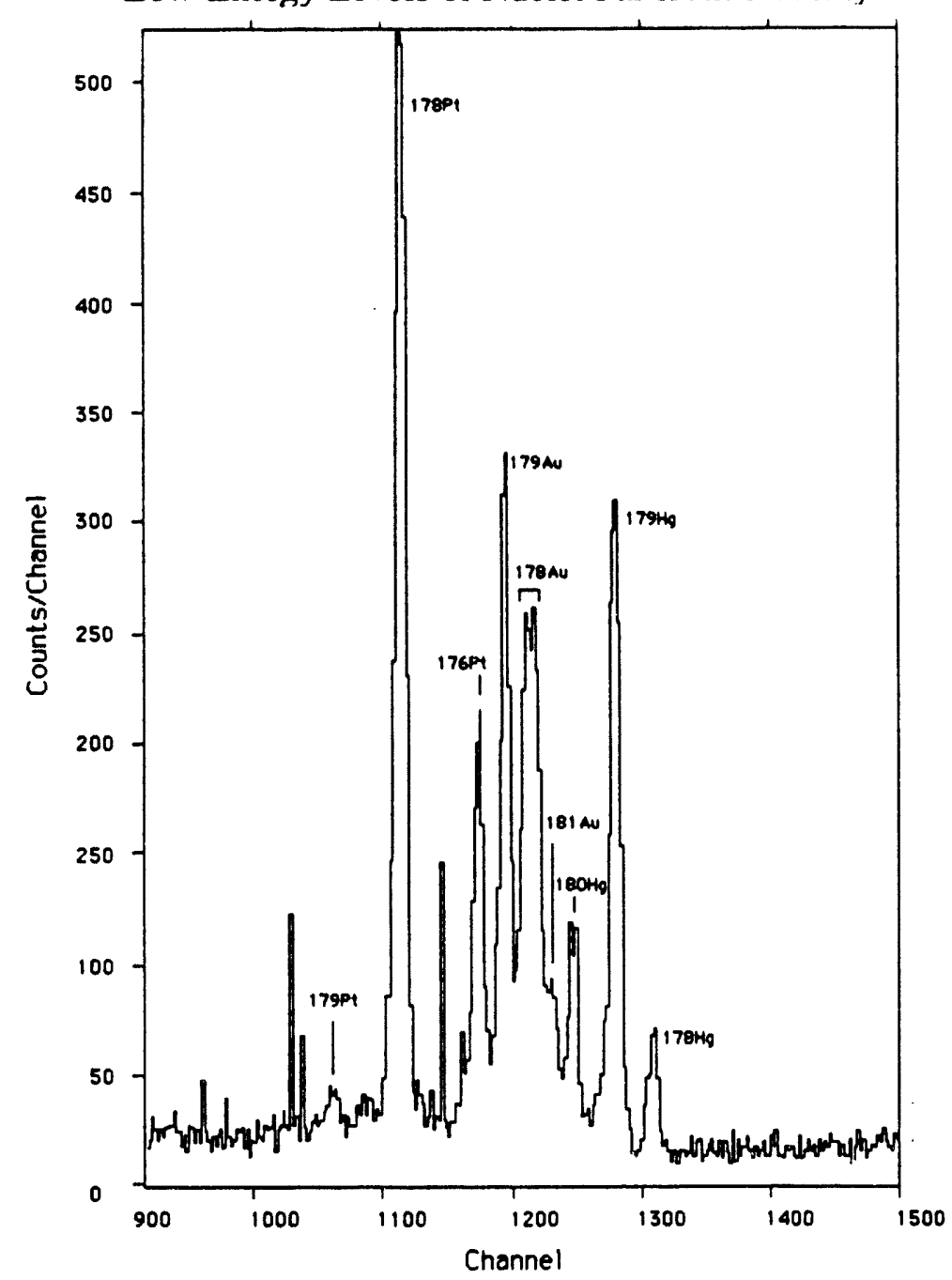

Figure II.B.2.1: Alpha decay spectrum of products of the reaction of $216 \mathrm{MeV}$ ${ }^{40} \mathrm{Ca}$ on ${ }^{144} \mathrm{Sm}$, summing over all 48 front strips in the DSSD. Central mass focussed on PPAC was $\mathrm{A}=179$.

correlations for mass 179 . The large group of counts in the middle of the spectrum correspond to decays following the decay of ${ }^{179} \mathrm{Hg}$, which would be ${ }^{175} \mathrm{Pt}$. Gating on the ${ }^{179} \mathrm{Hg}$ parent and projecting the $\mathrm{x}$-axis produces the spectrum in Figure II.B.2.4.

From this spectrum, the branching ratio $b_{\alpha}$ can be deduced for ${ }^{175} \mathrm{Pt}$. Taking the areas of the individual peaks and comparing to the total number of parent nuclei produced (found by calculating the area of the ${ }^{179} \mathrm{Hg}$ peak in the spectrum shown in Figure II.B.2.2) and correcting both for efficiency, the branching ratios for the transitions are 5.9(7)\%, 47.0(22)\%, and 8.9(9)\% respectively for the 5848-, 5966-, and $6029-\mathrm{keV}$ transitions. The total branching results are consistent with those 


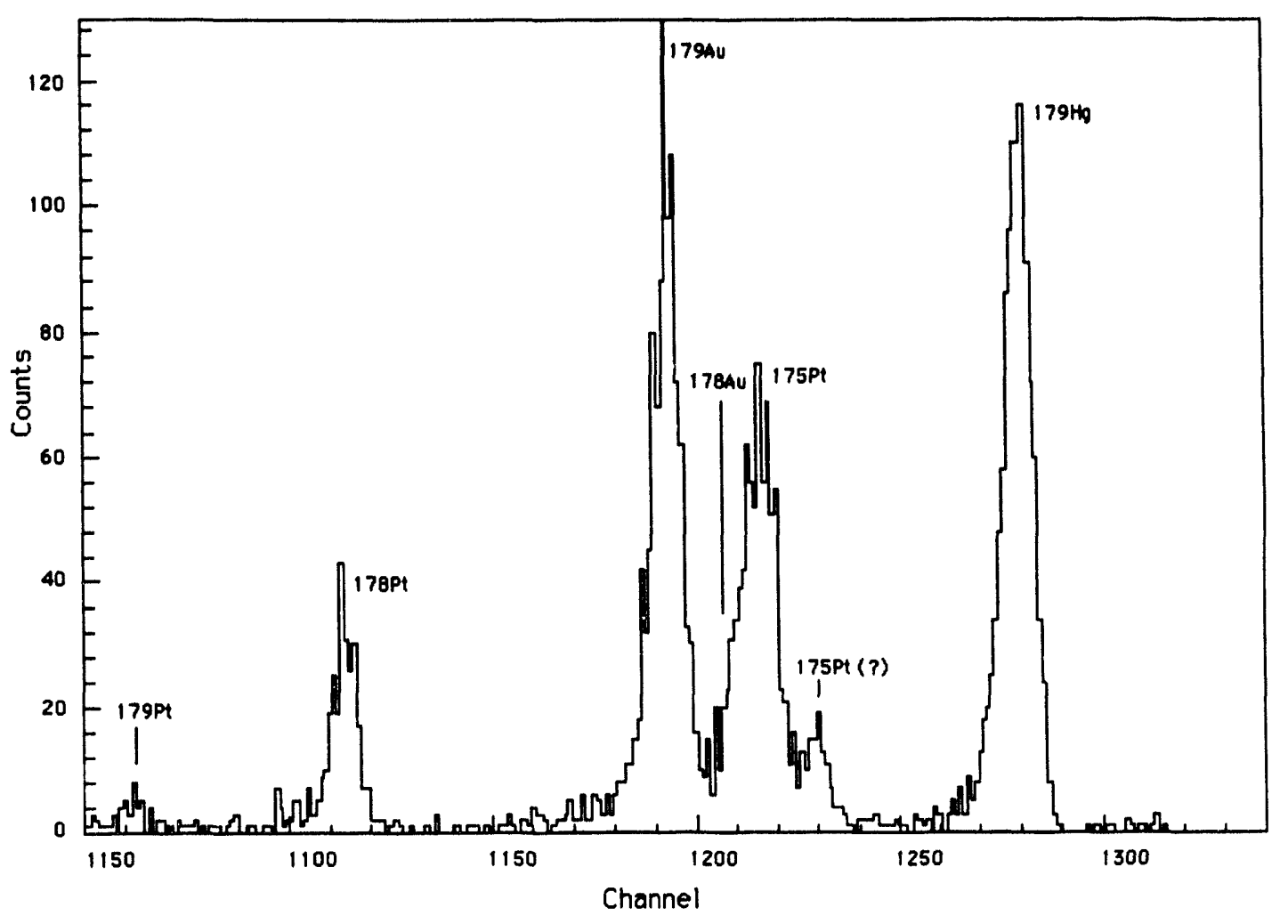

Figure II.B.2.2: Alpha decay spectrum of products of reaction of $216 \mathrm{MeV}^{40} \mathrm{Ca}$ on ${ }^{144} \mathrm{Sm}$, summing over all 48 front strips in the DSSD. Spectrum is gated with requirement that events must originate from a PPAC position corresponding to $A=179$ and that they are decay events (not implantations). 


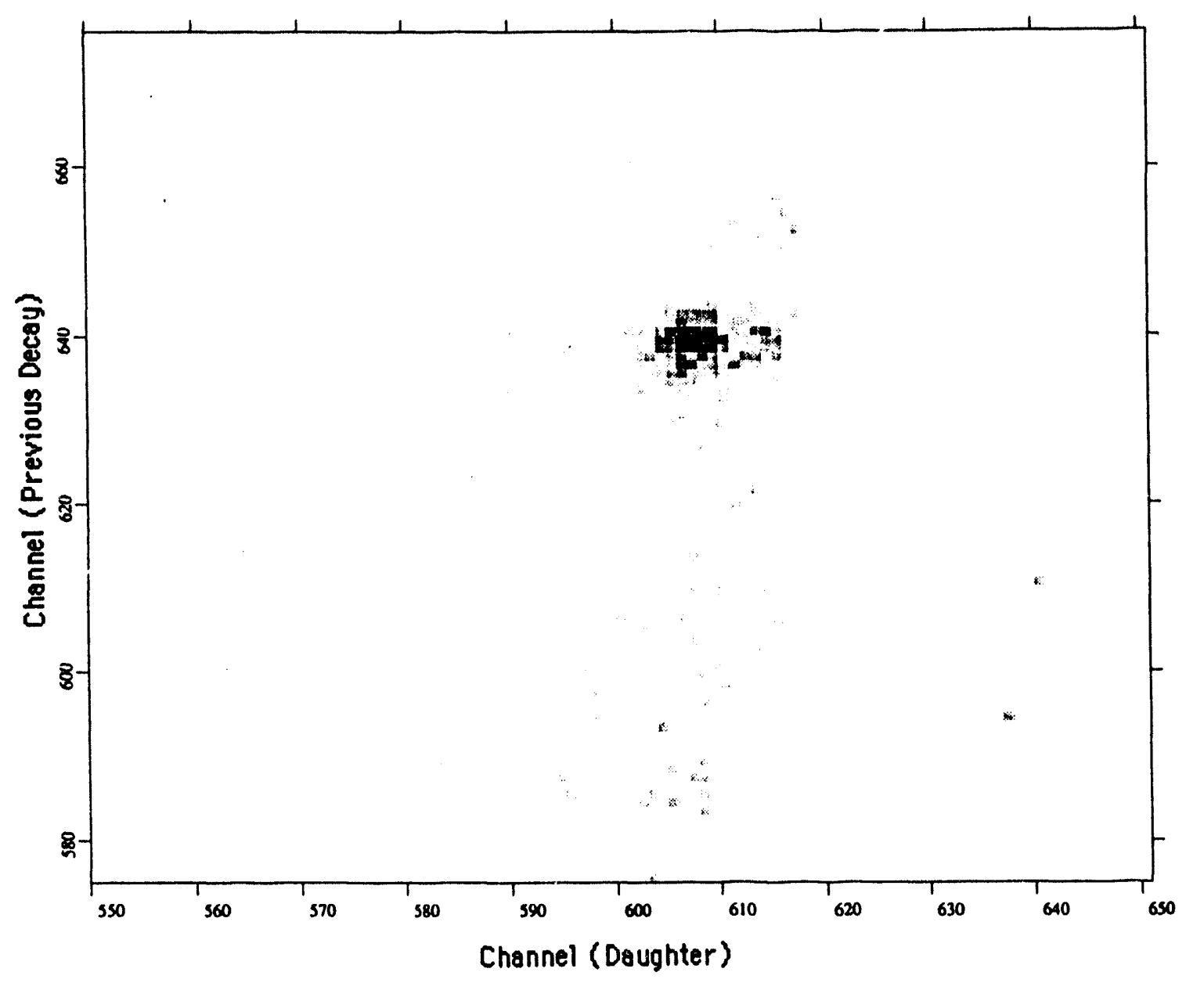

Figure II.B.2.3: Correlation spe trum of $A=179$ alpha decays. The $x$-axis is the spectrum of "daughter" nuclei and the y-axis is the spectrum of the alpha decays which preceed the corresponding alpha in the $\mathrm{x}$-axis in the same pixel. Events are mass-gated with $A=179$.

reported by Keller, et al. [8] and Hagberg, et al. [9]. However, the previous values are based only on the 5966- and 6029- keV alpha decays since the 5848- keV alpha is contaminated with the $5848-\mathrm{keV}$ alpha decay line from ${ }^{179} \mathrm{Au}$. In addition, the previous work reports that the decays to the $211-\mathrm{keV}$ and ground state levels in ${ }^{171} \mathrm{Os}$ are equal in intensity, which is not consistent with our results. Again, this is most likely due to contamination from ${ }^{179} \mathrm{Au}$, which the correlation analysis eliminates.

By creating histograms of energy versus time, time spectra can be created 
Alpha Decay Studies in $\mathrm{Hg}, \mathrm{Au}, \mathrm{Pt}$

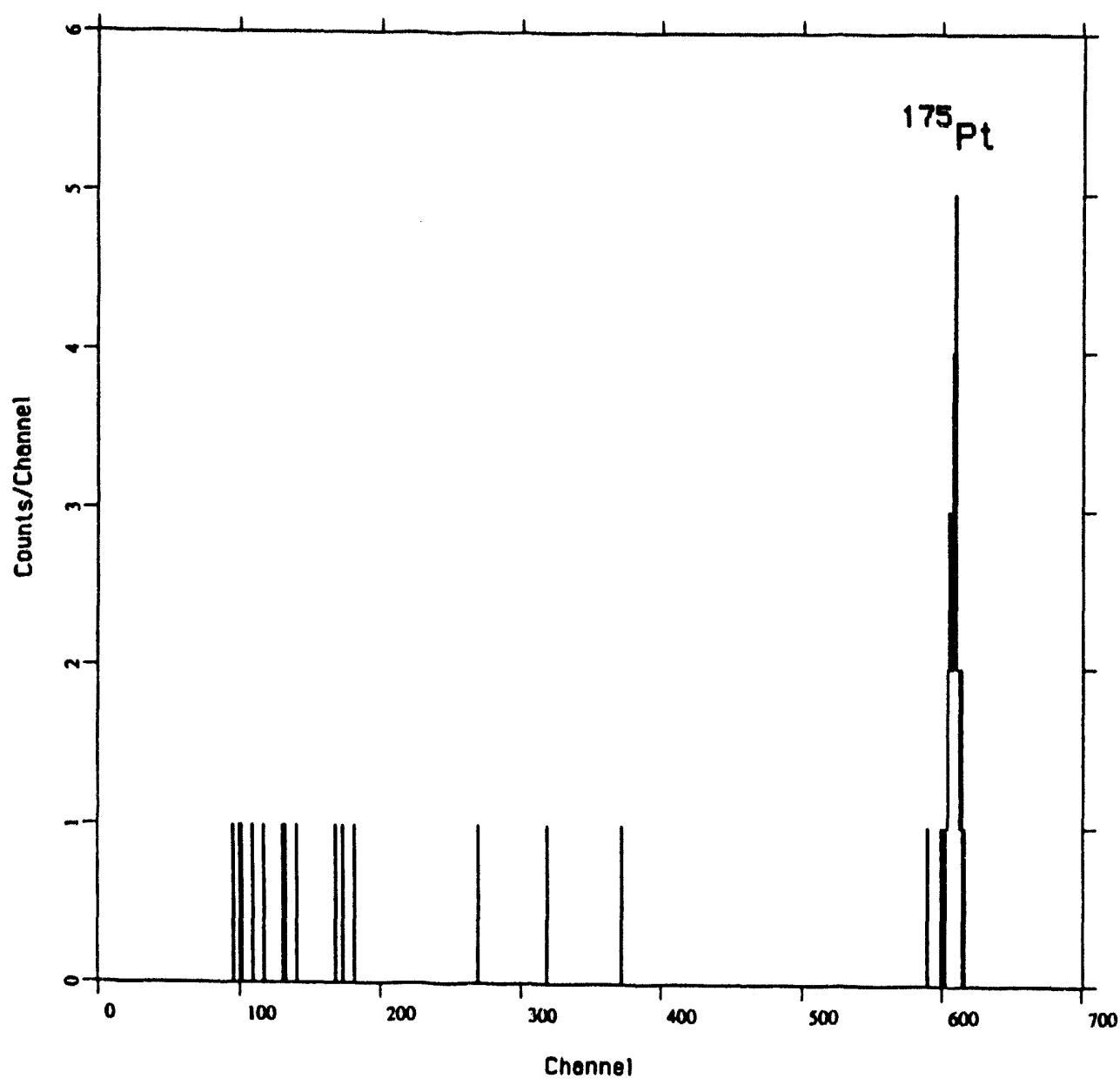

Figure II.B.2.4: Projection of the $x$-axis in Figure II.B.2.3, gating on the ${ }^{179} \mathrm{Hg}$ parent in the $y$-axis.

for all but the weakest decays. From those data, we performed linear, weighted least-squares fit to determine the halflife.

Preliminary results from this experiment are presented in Table II.B.2.1 and are all consistent with previously reported values. 
Table II.B.2.1: Preliminary results of alpha decays observed in the reaction of 216 $\mathrm{MeV}^{40} \mathrm{Ca}$ on ${ }^{144} \mathrm{Sm}$ using the DSSD

\begin{tabular}{llll} 
Energy (keV) & Nuclide & $t_{1 / 2}(s)$ & $b_{\alpha}(\%)$ \\
\hline $5168(56)$ & ${ }^{179} \mathrm{Pt}$ & & \\
$5443(21)$ & ${ }^{178} \mathrm{Pt}$ & $20.8(6)$ & \\
$5851(22)$ & ${ }^{179} \mathrm{Au}$ & $6.86(43)$ & \\
$5858(36)$ & ${ }^{178} \mathrm{Au}$ & & \\
$5930(28)$ & ${ }^{178} \mathrm{Au}$ & & \\
$5976(35)$ & ${ }^{178} \mathrm{Au}$ & & \\
$6120(22)$ & ${ }^{180} \mathrm{Hg}$ & $2.56(55)$ & \\
$6284(21)$ & ${ }^{179} \mathrm{Hg}$ & $1.06(8)$ & \\
$6437(40)$ & ${ }^{178} \mathrm{Hg}$ & $0.24(4)$ & \\
$6028(49)$ & ${ }^{181} \mathrm{Hg}$ & $4.00(17)$ & \\
$5752(26)$ & ${ }^{176} \mathrm{Pt}$ & $6.90(66)$ & $37.1(51)$ \\
$5848(25)$ & ${ }^{175} \mathrm{Pt}$ & & $5.9(7)$ \\
$5966(20)$ & ${ }^{175} \mathrm{Pt}$ & & $47.0(22)$ \\
$6029(24)$ & ${ }^{175} \mathrm{Pt}$ & & $8.9(9)$ \\
$6034(25)$ & ${ }^{174} \mathrm{Pt}$ & & $7.2(10)$ \\
\hline
\end{tabular}

\section{II.B.3. Proton Radioactivity Measurements on ${ }^{146,147} \mathrm{Tm}$ and ${ }^{160} \mathbf{R e}$}

Cary Davids, M. Freer, D. J. Henderson, R. Hermann, H. Penttila, A. H. Wuosmaa, [ANL], C. R. Bingham, J. D. Richards, B. E. Zimmerman, [UTK], L. Conticchio, W. B. Walters, [Maryland], A. V. Ramayya [Vanderbilt], P. Woods and R. Page [Edinburgh]

The first observation of proton radioactivity occurred about 20 years ago[10], but up to now only about 10 cases of proton radioactivity have been observed[11]. The paucity of data is no doubt related to the narrow $Q$ window for proton emission that results in successful competition with $\beta^{+}$decay and at the same time produces an observably long half-life. Also, the lack of suitable reactions to produce nuclei beyond the proton drip line adds to the complicity. The use of (HI,p3n) reactions at Daresbury coupled with the use or the RMS there resulted in the discovery of almost half of the known proton emitters in the last 4 years[12]. From systematic comparisons of their data with the results of mass formulas[13], it appears that the observation of proton radioactive decay will be possibie in many additional elements if the isotope 1 neutron further removed from stability than the lightest one studied 
by Livingston, et al.[14] at Daresbury can be produced. There is a good chance of doing this at Argonne, utilizing the FMA to separate fusion residues, and perhaps an improved chance when utilizing radioactive ion beams at a future RIB facility. Thus, we are collaborating on such experimentation at the FMA presently, and are making plans to utilize the RMS at Oak Ridge for similar studies when the HRIBF becomes operational in a few years.

There are several reasons for the interest in proton radioactivity. The actual decay characteristics of nuclei along the proton drip line is interesting. For example the details of the evolution of heavy nuclei in astrophysics is still in question, the network equations governing the buildup and decay of heavy nuclei require half-life information, and half-lives vary dramatically with neutron number at the onset of spontaneous proton emission. The proton decay energies are useful in the test of mass formulas and in the improvement of mass formulas by readjusting parameters to fit a wider range of masses. Since proton decay is a simple process, it is quite useful in testing what the Fermi level shell model orbitals are at the edge of stability, and information on nuclear potential shapes and strengths, including observation of the onset of deformation, may be possible with the provision of a broader range of data. A deeper theoretical understanding of quasibound and continuum wavefunctions and ways to couple these may result from a detailed description of this novel decay process.

Three runs have been made in the study of proton radioactivities at the FMA online to the ATLAS at Argonne National Laboratory. In the first commissioning run, a ${ }^{92} \mathrm{Mo}$ target was bombarded with $261-\mathrm{MeV}$ and $290-\mathrm{MeV}^{58} \mathrm{Ni}$ in order to emphasize the production of ${ }^{147} \mathrm{Tm}$ and ${ }^{146} \mathrm{Tm}$. Reaction products at $0^{\circ}$ were identified by $\mathrm{M} / \mathrm{q}$ with the FMA and implanted into a double-sided silicon strip detector (DSSD) located $27 \mathrm{~cm}$ behind the focal plane. The DSSD has 48 horizontal strips on the front and an equal number of vertical strips on the back from which the energy of the recoil and any subsequent charged particle decay can be read. In addition to the energy, the numbers of the strips that fire give the location in the detector of the recoil or decay event. The optics of the FMA allowed uniform illumination of the $1.6 \mathrm{~cm}$ by 1.6 $\mathrm{cm}$ DSSD allowing use of nearly all of the 2304 pixels as independent detectors in which to correlate recoil and decay events. Recoils were identified via coincidences between a parallel plate avalanche counter (PPAC) located in the focal plane and the DSSD and the time of arrival of a recoil in any pixel was recorded. Thus, the decay time and energy of any subsequent decay (a DSSD signal without a PPAC signal) in the same pixel could be determined. The implantation count rate of $60 \mathrm{~Hz}$ was maintained for about 24 hours with a 2 particle $n A$ bearn.

One of the benefits of correlating recoil and decay events is shown dramatically 


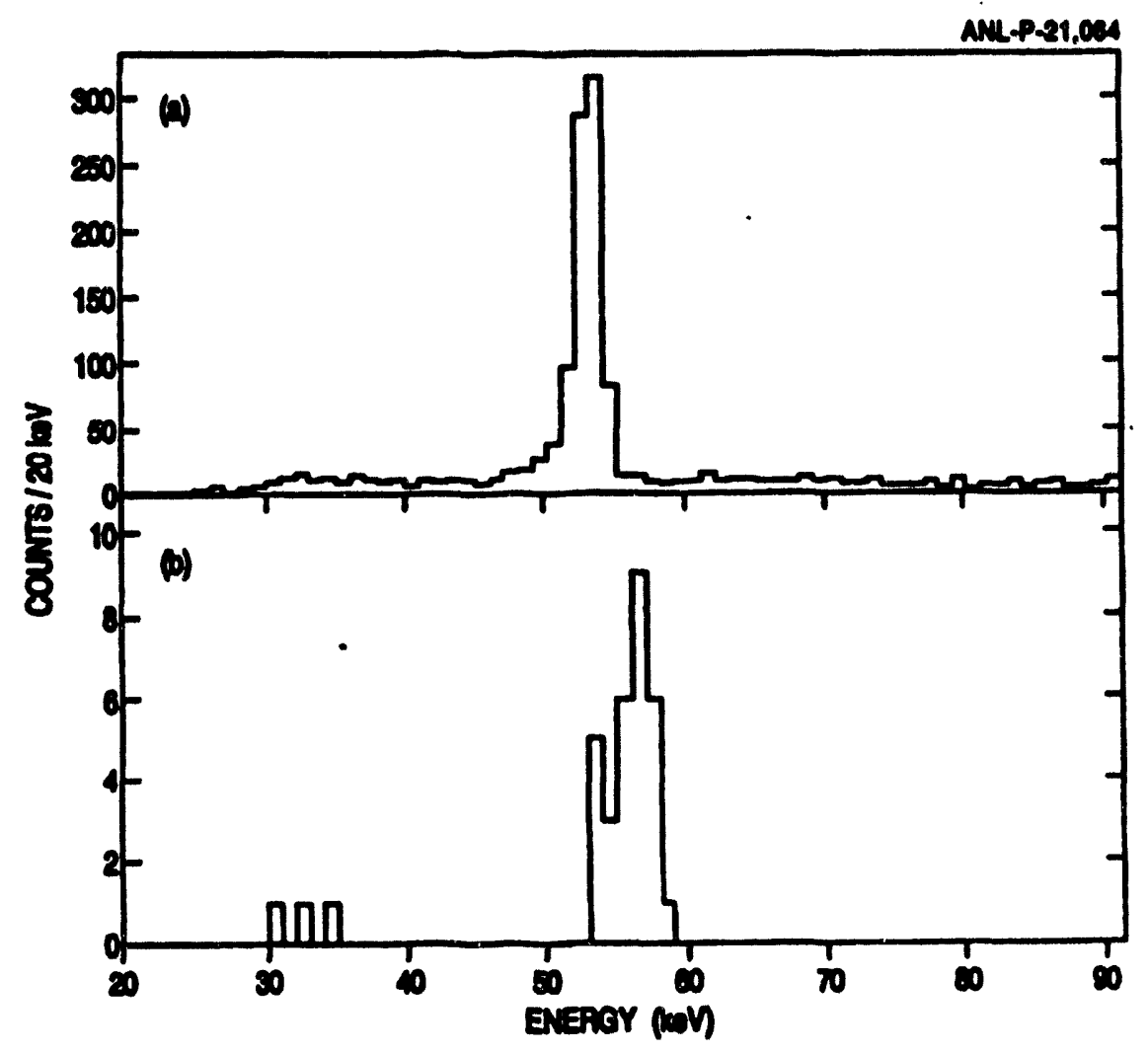

Figure II.B.3.1: Proton spectra of ${ }^{147} \mathrm{Tm}$ with time since recoil time a)between 10 $\mathrm{ms}$ and $5 \mathrm{~s}$, and $\mathrm{b}$ ) less than $10 \mathrm{~ms}$.

in Figure II.B.3.1. The spectrum in the upper part of the figure is for decay protons which were emitted by implants in the time interval between $10 \mathrm{~ms}$ and $5 \mathrm{~s}$, while the lower spectrum is for those emitted in less than $10 \mathrm{~ms}$ after deposit of the recoil. The upper spectrum is dominated by the relatively long-lived ${ }^{1478} \mathrm{Tm}$, whereas the lower has mainly decay protons from the much shorter lived ${ }^{147 \mathrm{~m}} \mathrm{Tm}$. Similar spectra were obtained for ${ }^{146} \mathrm{Tm}$ and though it is not certain that either of the proton emitting states is the ground state, we will refer to the two as ${ }^{146 g} \mathrm{Tm}$ and ${ }^{146 m} \mathrm{Tm}$. A total of 740 and 133 events were accumulated for the larger peaks corresponding to the decay of ${ }^{147 \mathrm{~g}} \mathrm{Tm}$ and ${ }^{1468 \mathrm{Tm}} \mathrm{T}$, respectively. These counts are displayed as a function of decay time after recoil in Figure II.B.3.2, where least-squares fits of the data are also displayed. The fitted half-lives are $559 \pm 26 \mathrm{~ms}$ and $206 \pm 25 \mathrm{~ms}$ for ${ }^{147} \mathrm{~g} \mathrm{Tm}$ and ${ }^{1468} \mathrm{Tm}$, respectively. The maximum likelihood method was applied to $23{ }^{147 \mathrm{~m}} \mathrm{Tm}$ and $15^{146 m} \mathrm{Tm}$ events to obtain halflives of $390_{-71}^{+93} \mu \mathrm{s}$ and $62_{14}^{+19} \mu \mathrm{s}$, respectively.

The half-lives obtained in this first commissioning run are compared with 

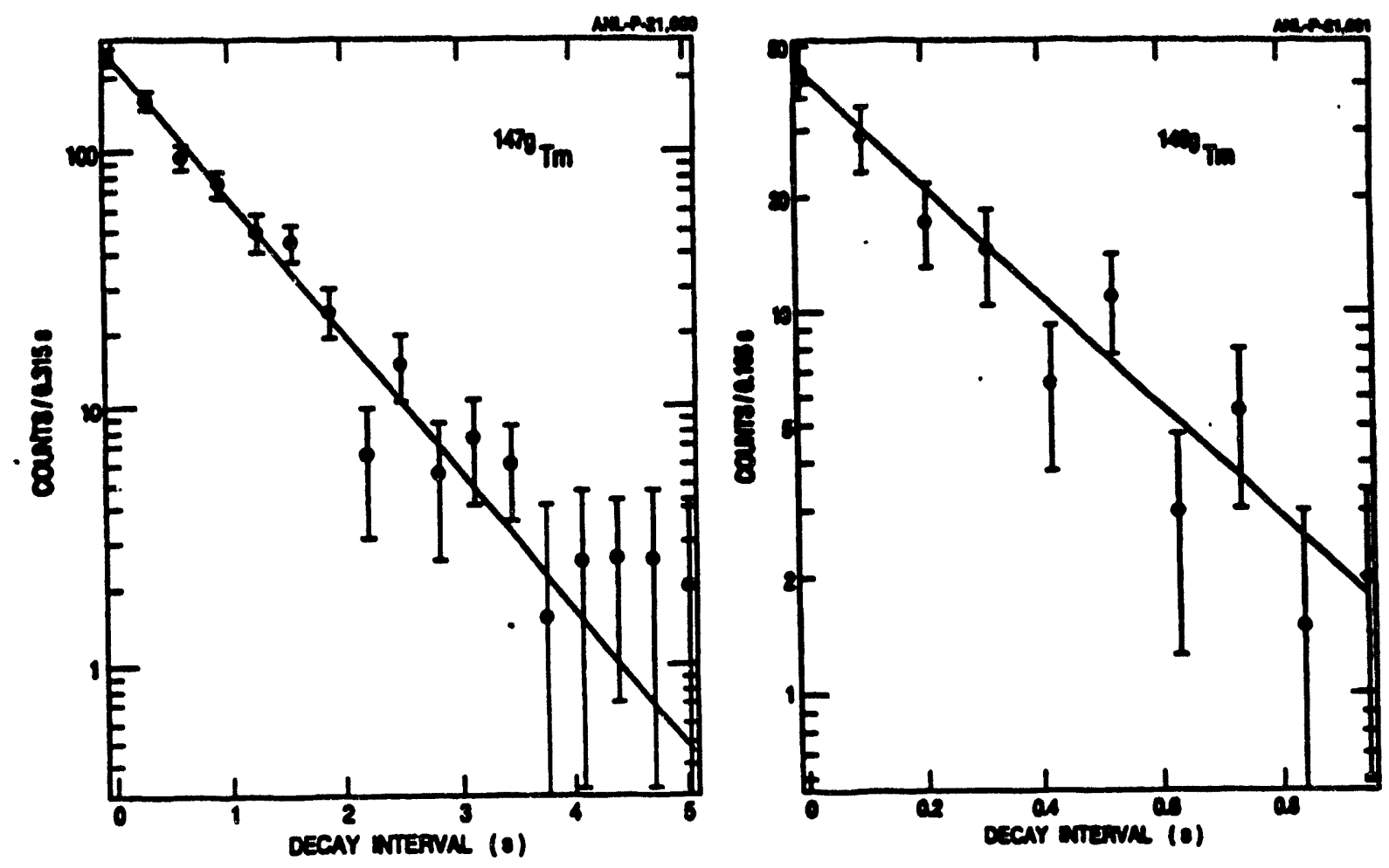

Figure II.B.3.2: Decay curves for ${ }^{147 g} \mathrm{Tm}$ and ${ }^{146 g} \mathrm{Tm}$.

previous results [15, 7] in Table II.B.3.1, and also, with the results of a WKB calculation [15, 7] of the barrier penetration with various orbitals near the Fermi level in this region of the periodic chart. The half-life results are seen to agree well with the Daresbury results as shown in the table, and for the case of ${ }^{147 \mathrm{~g}} \mathrm{Tm}$ and ${ }^{147 m} \mathrm{Tm}$, with earlier values[16] of $560 \pm 40$ and $0.36 \pm 0.08 \mathrm{~ms}$, respectively. The calculated half-life for the $h_{11 / 2}$ orbital is in almost perfect agreement with the experimental value for ${ }^{146 \mathrm{~m}} \mathrm{Tm}$. The half-life of ${ }^{146 \mathrm{~g}} \mathrm{Tm}$ is also only consistent with the calculation for the $h_{11 / 2}$ orbital, but in this case the competition of $\beta^{+}$decay is effective in reducing the half-life. The half-life for the ${ }^{1478} \mathrm{Tm}$ is consistent with only the $h_{11 / 2}$ orbital since large hindrance factors are not expected for proton decay in this region; in this case the $\beta^{+}$branch would be about $80 \%$ and the proton branch about $20 \%$. The half-life for ${ }^{147 \mathrm{~m}} \mathrm{Tm}$ has been cited[7] as evidence that it is dominantly a $d_{3 / 2}$ orbital; it is apparent that the hindrance factor is about 2.6 , a reasonable number.

In a recent run at the FMA ${ }^{106} \mathrm{Cd}$ was bombarded with ${ }^{58} \mathrm{Ni}$ to produce ${ }^{160} \mathrm{Re}$, the heaviest known ground-state proton emitter. While the proton radioactivity was 
Table II.B.3.1: Comparison of proton energies and decay half-lives from the present experiment with earlier results and with the results of WKB calculations with different proton orbitals.

\begin{tabular}{llllllll}
$\begin{array}{l}\text { Proton } \\
\text { emitter }\end{array}$ & \multicolumn{2}{c}{ Half life } & & \multicolumn{3}{c}{ WKB Calc. Half Life } \\
& $\frac{\text { Present }}{206 \pm 25}$ & $\frac{\text { Previous }}{235 \pm 27}$ & $\frac{\text { Ref. }}{[15]}$ & $\frac{h_{11 / 2}}{374}$ & $\frac{d_{3 / 2}}{0.12}$ & $\frac{s_{1 / 2}}{0.013}$ \\
${ }^{146 m} \mathrm{Tm}$ & $62_{-14}^{+19}$ & $72 \pm 23$ & {$[15]$} & 58 & 0.019 & 0.002 \\
${ }^{147 g} \mathrm{Tm}$ & $559 \pm 20$ & $560 \pm 40$ & {$[7]$} & 2700 & 0.86 & 0.095 \\
${ }^{147 m} \mathrm{Tm}$ & $0.390_{-0.071}^{+0.093}$ & $0.36 \pm 0.04$ & {$[7]$} & 460 & 0.150 & 0.017
\end{tabular}

observed, detailed analysis of these data is still in progress.

\section{II.B.4. Decay of Mass-Separated ${ }^{114} \mathrm{Te}$}

B. E. Zimmerman [Maryland/UTK]; W. B. Walters [Maryland]; P. F. Mantica, Jr., H. X. Carter, and J. Kormicki [UNISOR]; Z. Gácsi and Zs. Dombrádi[ATOMKI, Debrecen]

Systematic experimental investigations of light odd-odd Sb nuclei $[17,18,19]$ performed by the Debrecen group have been successful in extending or improving their level schemes and has provided a basis for theoretical calculations in the Interacting Boson- Fermion-Fermion Model (IBFFM). The calculations will hopefully provide a better understanding of the interaction between protons and neutrons and how this interaction affects the states that result from the coupling of the odd particles. To aid in extending this study, we have investigated the structure of energy levels populated in the $\beta^{+} / \mathrm{EC}$ decay of ${ }^{114} \mathrm{Te}\left(\mathrm{t}_{1 / 2}=15 \mathrm{~m}\right)$ using the mass separator at UNISOR.

Products of the reaction of $230 \mathrm{MeV}{ }^{32} \mathrm{~S}$ on a ${ }^{92} \mathrm{Mo}$ target were separated by mass and deposited in a mylar tape which could be moved sequentially to two shielded counting stations. Multiscaled $\gamma$ singles, normal electron singles, and $\gamma-$ $\gamma$ and electron $\gamma$ coincidences were collected. These data, together with those collected from a recent experiment at Debrecen using the $(\mathrm{p}, \mathrm{n} \gamma)$ reaction, allowed for the assignment of many new transitions, internal conversion coefficients, levels, and spins.

Subsequent to reporting our preliminary results last year, we have undertaken 


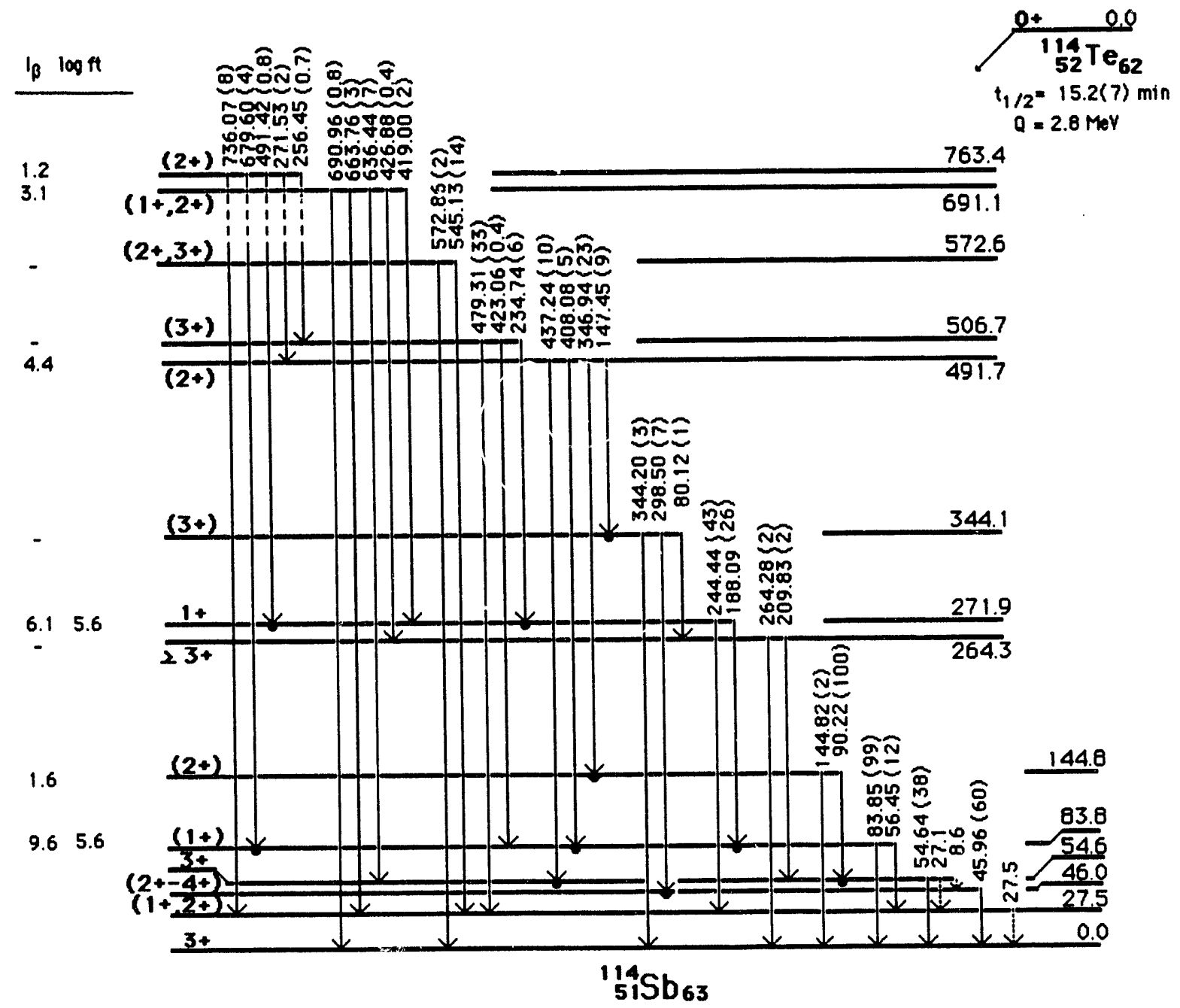

Figure II.B.4.1: Low-energy (levels below $800 \mathrm{keV}$ ) portion of level scheme observed in the decay of $15 \mathrm{~m}{ }^{114} \mathrm{Te}$. The log $\mathrm{ft}$ values were only calculated for levels receiving more than $5 \%$ of the total decay feeding.

a re-analysis of the data to resolve differences between our results and those from Debrecen. There is now excellent agreement between the two experiments.

Figure II.B.4.1 shows the low-energy portion of the present level scheme. One of the new results of the re-analysis has been the determination of the halflives of the 54- and 45-keV transitions. Time spectra are shown in Figure II.B.4.2 for the 45- and 54-keV transitions, along with the $84-$ and $90-\mathrm{keV}$ prompt transitions for reference. From these data, the halflives were found to be 25.8(11) ns and 20.4(70) 

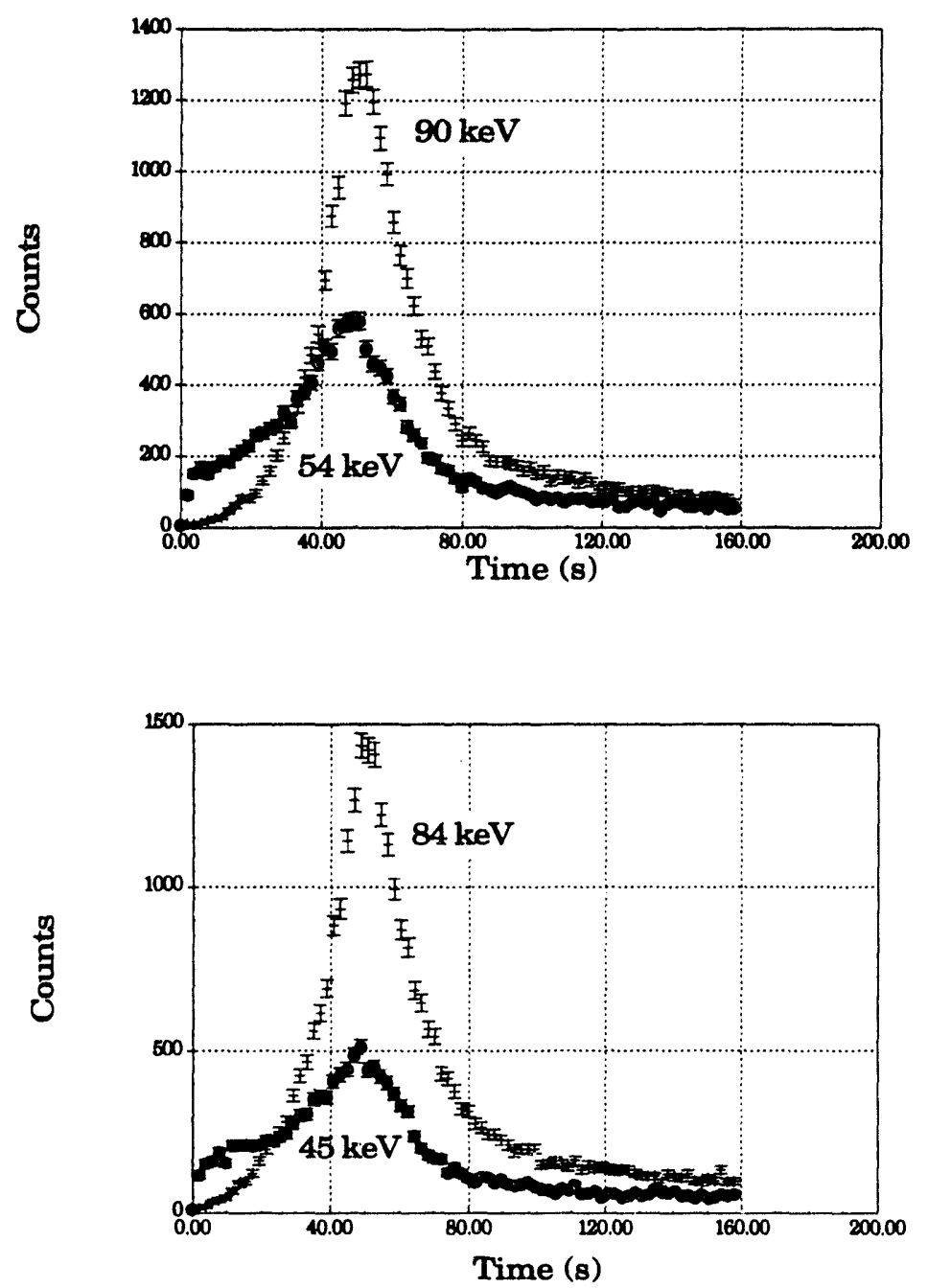

Figure II.B.4.2: Time spectra gated by the 83-, 90- (prompt) and 45-, and 54(delayed) $\mathrm{keV}$ transitions, requiring a coicidence with any other gamma ray.

ns resepctively for the 45- and 54-keV transitions and agree well with the upper limit of 24(5) ns reported Wigmans, et al. [19].

Final theoretical calculations are being performed and the manuscript for the paper reporting both the experimental and theoretical results is being prepared for publication. 


\section{II.B.5. Decay of Mass-Separated ${ }^{199} \mathrm{Bi}$ and ${ }^{193} \mathrm{Bi}$}

M. Zhang, C. R. Bingham, J. Ding, B. E. Zimmerman, J.-Y. Zhang [UTK]; H. K. Carter, J Kormicki, P. F. Mantica, Jr., and L. Rayburn [UNISOR]; P. Joshi [LSU]; J. L. Wood [Ga. Tech.]; S. Shastry [SUNY-Plattsburgh]

The $\beta$-decay of ${ }^{199} \mathrm{Bi}$ isotope and the study of shape coexistence in odd- $\mathrm{A}$ $\mathrm{Pb}$ isotopes was reported in last year's annual progress report, in which we have discussed the level scheme of ${ }^{199} \mathrm{~Pb}$, E0 transitions and level systematics of the deformed $\mathrm{O}_{2}^{+}$states in even-even $\mathrm{Pb}$ nuclides along with the deformed $13 / 2^{+}$states in odd- $\mathrm{A} \mathrm{Pb}$ nuclides. Using the deformed Nilsson potential, we calculated the excitation energies of lower single particle states for odd- $\mathrm{A} \mathrm{Pb}$ nuclides within $A=193-205$. The energy difference between the theoretical and experimental values of the excitation energy of the $p_{1 / 2}$ state was large. Also, we had not observed the low lying $\mathrm{p}_{1 / 2}$ state in ${ }^{199} \mathrm{~Pb}$.

We re-investigated the results of ${ }^{199} \mathrm{Bi} \beta$-decay, especially the $\gamma-\gamma$ and $\gamma$-e coincidence spectra. More transitions were placed in the level scheme and several new levels were added to the scheme. One of the new results was a $216 \mathrm{keV} \mathrm{M} 1$ transition to the $3 / 2^{-}$ground state, creating a new state which is $(1 / 2,3 / 2,5 / 2)^{-}$. Most of new levels below $1400 \mathrm{keV}$ are connected with this new low-spin negative parity state. The new level scheme for ${ }^{199} \mathrm{~Pb}$ is shown in Figure II.B.5.1.

The decay of ${ }^{193} \mathrm{Bi}$ was also studied utilizing the University Isotope Separator at Oak Ridge (UNISOR) on-line to the Holifield Heavy-Ion Research Facility tandem accelerator. From multiscaled $\gamma$-ray and conversion electron singles data, as well as $\gamma-\gamma$ and $\gamma$-electron coincidences, 53 transitions were placed in a 42 excited states level scheme (Figs. II.B.5.2 and II.B.5.3). The two lowest levels were taken to be $3 / 2^{-}$and $5 / 2^{-}$levels in analogy to the heavier odd $\mathrm{A} \mathrm{Pb}$ isotopes. These assignments are supported by the $\mathrm{M} 1$ character of the $175-\mathrm{keV}$ transition. One 354-keV M1 transition decayed to $\left(3 / 2^{-}\right)$ground state, also establishing a $(1 / 2,3 / 2$, $5 / 2)^{-}$state.

The experimental energies and $\mathrm{I}^{\pi}$ of the lowest 3 negative parity states of isotopes between ${ }^{193} \mathrm{~Pb}$ and ${ }^{205} \mathrm{~Pb}$ are displayed in Figure II.B.5.4, except for the case of ${ }^{197} \mathrm{~Pb}$ where the third one is still unknown. The $3 / 2^{-}$and $5 / 2^{-}$levels exchange their order around 199. The $1 / 2^{-}$starts at quite low excitation in ${ }^{205} \mathrm{~Pb}$ and increases for decreasing $\mathrm{A}$ to ${ }^{201} \mathrm{~Pb}$; the $\left(1 / 2^{-}, 3 / 2^{-}, 5 / 2^{-}\right)$levels of ${ }^{199} \mathrm{~Pb},{ }^{195} \mathrm{~Pb}$ and ${ }^{193} \mathrm{~Pb}$ follows the trend established in ${ }^{205-201} \mathrm{~Pb}$, and thus on that basis, they probably are the $1 / 2^{-}$levels in these nuclei. However, theoretical calculations of the energies of the $p_{3 / 2}, f_{5 / 2}$, and $p_{1 / 2}$ levels in these nuclei have been made using a deformed Nilsson potential,

$$
V=-\kappa h \omega_{0}\left[2 * 1 \cdot s+\mu\left(l_{t}^{2}-\left\langle l_{t}^{2}\right\rangle\right)\right]
$$




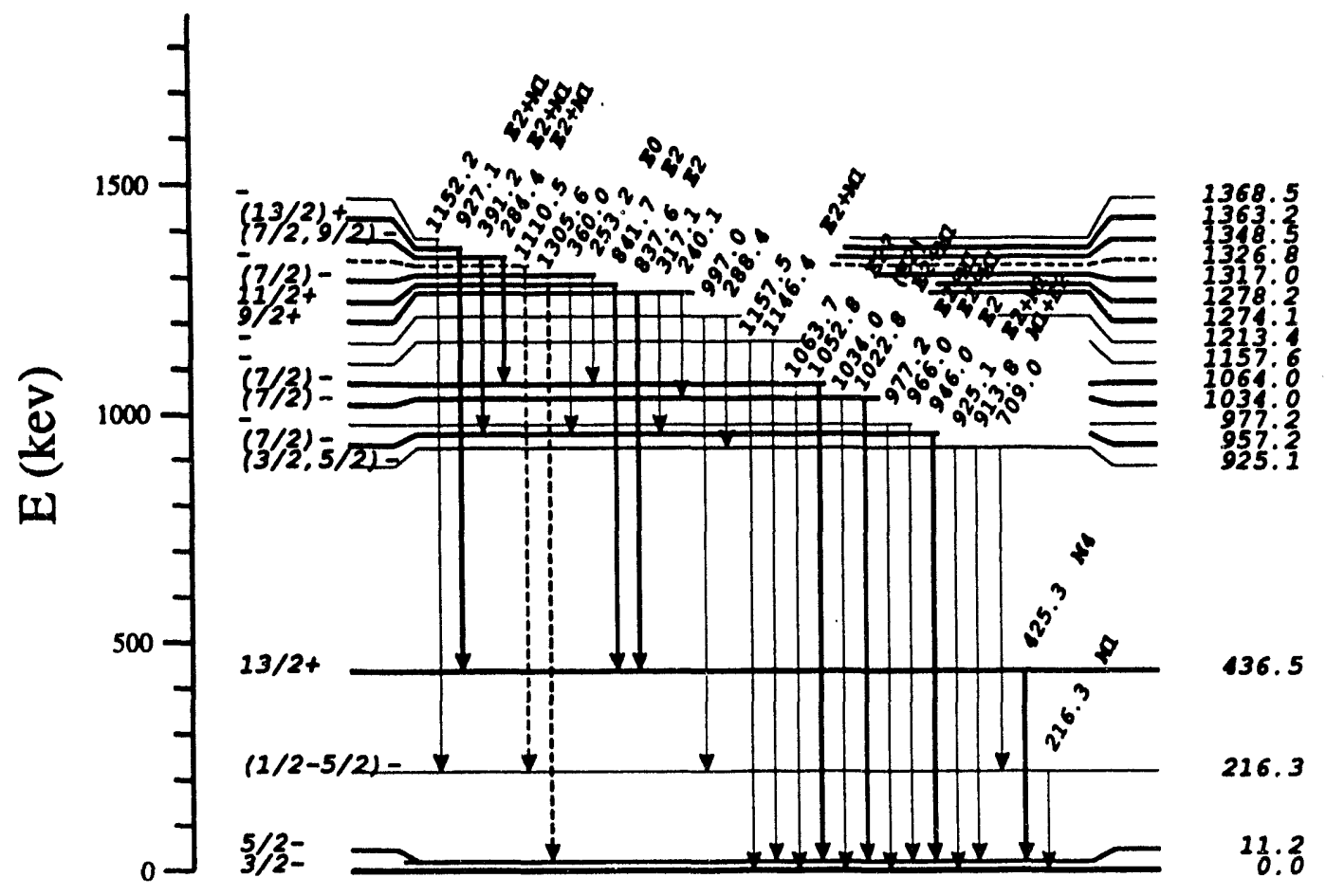

Figure II.B.5.1: Low energy states of ${ }^{199} \mathrm{~Pb}$. New levels and transitions observed during the last year are shown as bold lines.

with standard values [20] of the parameters $\kappa$ and $\mu$. As seen in Figure II.B.5.4 the predicted levels follow the trend of the experimental levels for the $3 / 2^{-}$and $5 / 2^{-}$levels, with excitation energy differences less than about $100 \mathrm{keV}$. Yet if the third negative parity levels of ${ }^{193,195,199} \mathrm{~Pb}$ are assigned to the $\mathrm{p}_{1 / 2}$ state, the deviations between experimental and calculated excitation energies are much greater, becoming about $600 \mathrm{keV}$ in ${ }^{193} \mathrm{~Pb}$. Small alteration of $\kappa$ and $\mu$ do not significantly alter the discrepancy.

\section{II.B.6. Search for $I=0$ Superdeformed Isomers in Non-actinide Nuclides: ${ }^{194} \mathrm{Hg}$}

W. B. Walters and L. F. Conticchio [Maryland]; B. E. Zimmerman, C. R. Bingham, and J. D. Richards[UTK]; P. F. Mantica, Jr. [ORISE]; T. Rinkel and C. Foster [IUCF]; P. K. Joshi [LSU]

The existence of isomers with large prolate $(\epsilon \sim 0.6)$ deformation in the 


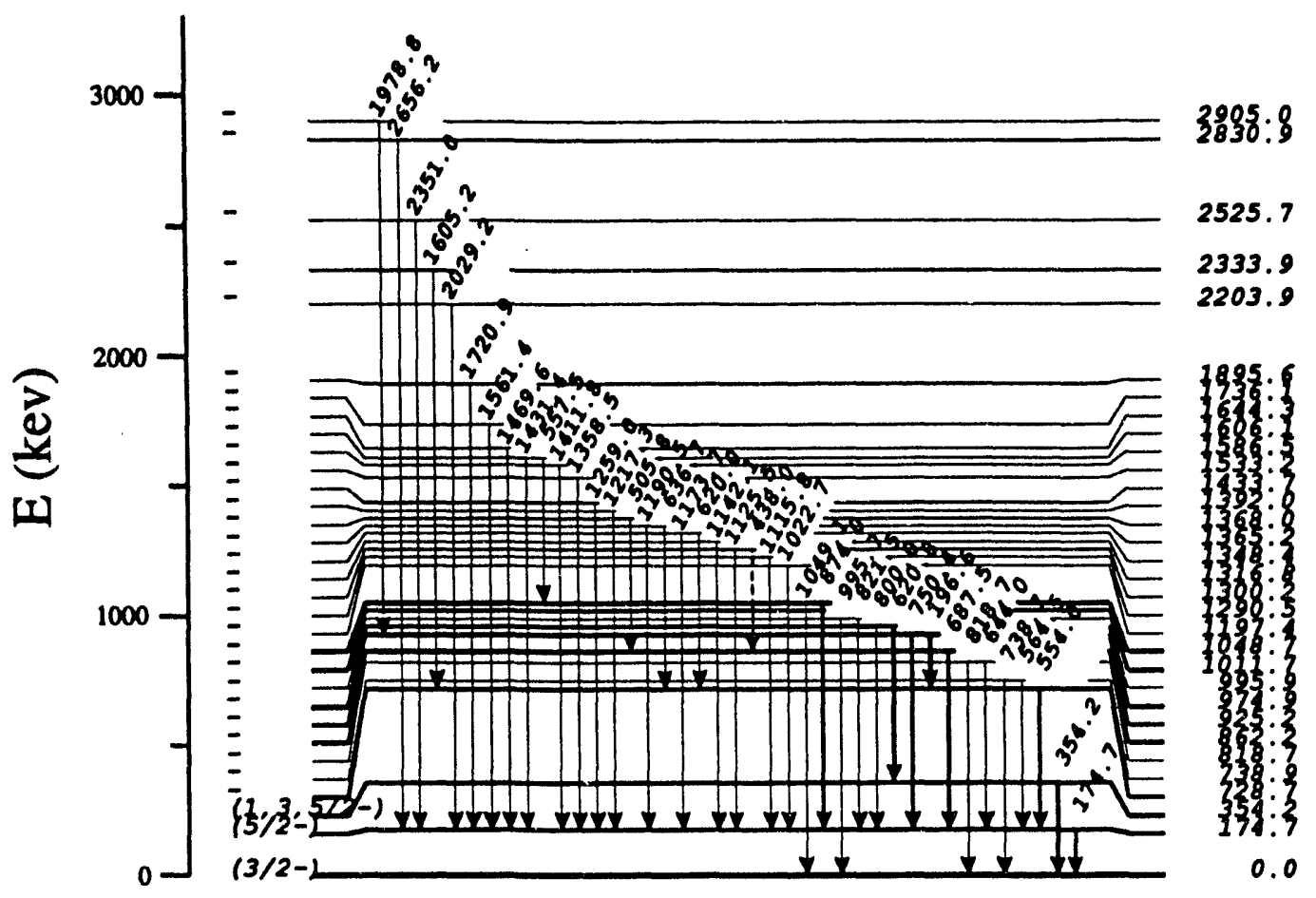

Figure II.B.5.2: Negative parity states of ${ }^{193} \mathrm{~Pb}$ and transitions observed in the $\beta^{+} /$EC decay of ${ }^{193} \mathrm{Bi}$.

actinide region has been known for several years and was established on the basis of observation of their spontaneous fission. Similar structures have been observed in the rare earth region near ${ }^{152} \mathrm{Dy}$ and in the $\mathrm{A}=135$ and $\mathrm{A}=190$ regions $[21,22,23]$. These bands were established through observation of rotational bands with high angular momentum. However, the exact nature of these bands have not been fully characterized, since they tend to depopulate out of the band at relatively high spin and do not decay to the bandhead. Therefore, the excitation energy of the bandhead (and thus the position of the superdeformed minimum) cannot be determined from these typical high-spin experiments.

Calculations $[22,24]$ have predicted the existence of superdeformed minima in the $A=190$ region and suggest that this second minimum is best developed as the number of neutrons $\mathrm{N}$ increases in the region where superdeformed bands have been previously observed. Recent calculations by Meyer, et al. [25] suggest that the best chance to observe $0^{+}$superdeformed isomers is in ${ }^{194} \mathrm{Hg}$, in which the superdeformed 


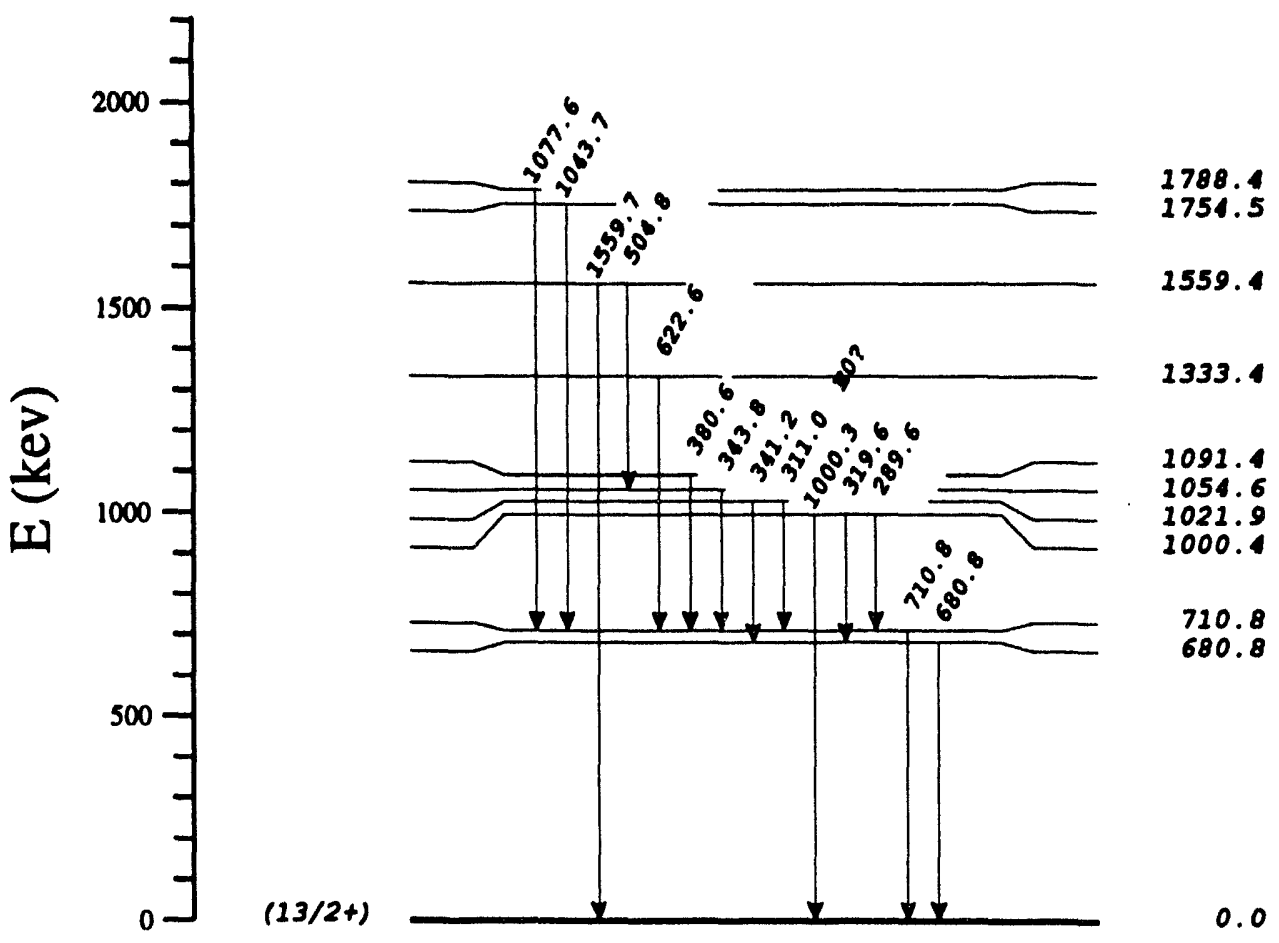

Figure II.B.5.4: Comparison of energies of the three lowest negative parity states of ${ }^{193} \mathrm{~Pb}-{ }^{205} \mathrm{~Pb}$ with predictions of a calculation using a Nilsson potential.

to look for a delayed component in the $\mathrm{BaF}_{2}-\mathrm{rf} \mathrm{TAC}$. This delayed component would be the signature of an isomeric transition which could depopulate the superdeformed isomeric state, and which feeds the $428-\mathrm{keV}$ gamma ray.

Preliminary analysis performed on-line during the experiment indicates that there is a delayed component in the $\mathrm{BaF}_{2}-\mathrm{rf} \mathrm{TAC}$ of $\leq 3 \mathrm{~ns}$, with the isomeric transition having an energy in the $3-4 \mathrm{MeV}$ energy range. Further analysis is required to sum all of the data and recalculate the delay in the TAC. However, the present results suggest that there is a $3-4 \mathrm{MeV}$ transition depopulating an isomeric state. In order to determine whether or not the state is the bandhead of a superdeformed band, it will be necessary to not only accurately determine the energy of the depopulating transition (by using $\mathrm{a}$ Ge detector in conjunction with the $\mathrm{BaF}_{2}$ detectors), but to observe transitions which in turn populate the isomeric transition. 


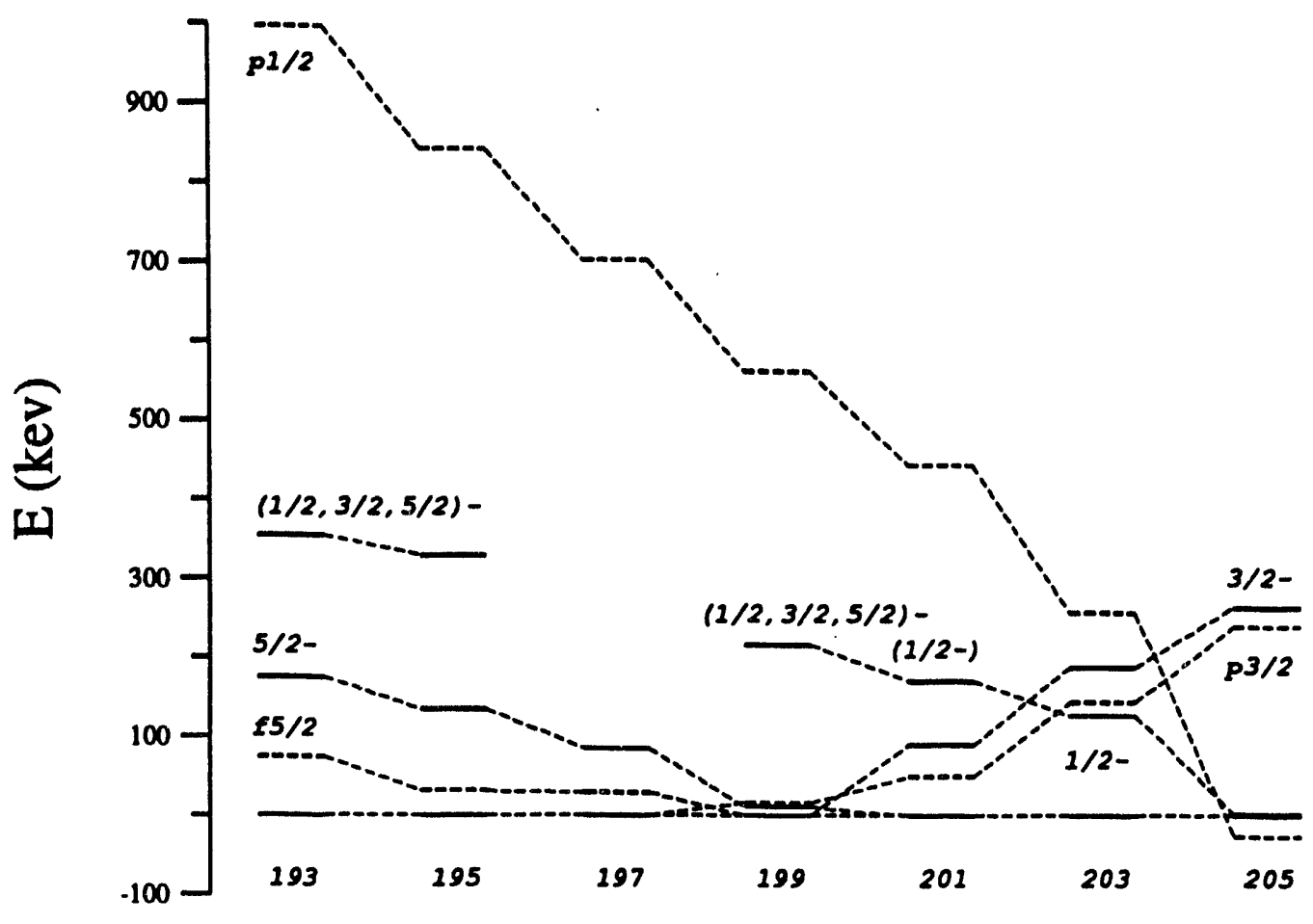

Figure II.B.5.3: Positive parity states of ${ }^{193} \mathrm{~Pb}$ and transitions observed in the $\beta^{+} / \mathrm{EC}$ decay of ${ }^{193} \mathrm{Bi}$. The excitation energy of the lowest positive parity $\left(13 / 2^{+}\right)$ is unknown and is labeled with the energy $E$.

bands depopulate at "very low spin". This nucleus was thus chosen as the starting point for a series of searches for such states in this mass region.

To begin this project, a preliminary experiment was performed at the Indiana University Cyclotron Facility (IUCF), in which a $45-\mathrm{MeV}$ beam of protons bombarded a $8.2 \mathrm{mg} / \mathrm{cm}^{2}$ target of ${ }^{197} \mathrm{Au}$. This provided population of high-energy, low-spin states in the $4 \mathrm{n}$ product channel, ${ }^{194} \mathrm{Hg}$. The key of the experiment was to bring as little angular momentum into the compound nucleus as possible, while providing sufficient excitation energy. The detector setup consisted of a pair of $\mathrm{BaF}_{2}$ detectors placed at the target position. Energy data were collected in each detector, as were time-to-amplitude (TAC) data between 1) the two $\mathrm{BaF}_{2}$ detectors and 2) the cyclotron rf pulse (approx. every $100 \mathrm{~ns}$ ) and one of the $\mathrm{BaF}_{2}$ detectors. By gating on the prompt 428-keV $2_{1}^{+} \rightarrow 0_{1}^{+}$transition, the prompt portion of the $\mathrm{BaF}_{2}-\mathrm{BaF}_{2}$ $\mathrm{TAC}$, and another energy range in the other $\mathrm{BaF}_{2}$ detector, it would then be possible 
[1] J. Wauters, et al., Proc. Int. Conf. on Atomic Masses and Fundamental Constants, BernkastelKuess 595 (1992).

[2] J. Wauters, et al., Z. Phyzik, accepted for publication.

[3] M. J. Martin, et al., Nucl. Data Sheets 61, 352 (1990).

[4] J. O. RasmusseN, et al., Phys. Rev. 113, 1593 (1959).

[5] R. Bengtsson, et al., Phys. Lett. B183, 1 (1987).

[6] J. L. Wood, et al., Phys. Rep. 215, 103 (1992).

[7] P. J. Sellin, et al., Nucl. Inst. Meth. A311, 217 (1992).

[8] J. G. Keller, et al., Nucl. Phys. A452, 173 (1986).

[9] E. Hagberg, et al., Nucl. Phys. A318, 29 (1979).

[10] Jackson, et al., Phys. Lett. 33B, 284 (1970).

[11] S. Hoffman, to be published in Handbook of Nuclear Decay Modes, CRC Press (1993).

[12] P. J. Woods, et al., Proc. 6th Int. Conf. on Nuclei Far From Stability \& 9th Int. Conf. on Atomic Masses and Fundamental Constants, Bernkastel-Kues 323 (1992).

[13] S. Liran and N. Zeldes, At. Data Nucl. Data Tables 17, 431 (1976).

[14] K. Livingston, et al., to be published .

[15] K. Livingston, et al., Phys. Lett. B 312, 46 (1993).

[16] S. Hoffman, et al., Proceedings of the 7th Int. Conf. on Atomic Masses and Fundamental Constants, AMCO-7, Darmstadt 184 (1984).

[17] Z. Gacsi, T. Feynes, Z. Dombradi, S. Brant, and V. Paar, Phys. Rev. C44, 642 (1991).

[18] J. Gulyas, T. Feynes, M. Fayez, F. M. Hassan, Zs. Dombradi, J. Kumpulainen, and R. Julin, Phys. Rev. C46, 1218 (1992).

[19] M. E. J. Wigmans, R. J. Heynis, P. M. A. van der Kam, and H. Verheul, Phys. Rev. C14, 243 (1976).

[20] T. Bengtsson and I. Ragnarsson, Nucl. Phys. A436, 14 (1985).

[21] P. J. Twin, et al., Phys. Rev. Lett. 57, 811 (1986).

[22] P. R. Chasman, Phys. Lett. B219, 227 (1989).

[23] R. V. F. Janssens and T. L. Khoo, Annu. Rev. Nucl. Part. Sci. 41, 321 (1991).

[24] W. Satula, et al., Nucl. Phys. A529, 289 (1991).

[25] J. Meyer, et al., Nucl. Phys. A533, 307 (1991). 


\section{II.C. High Energy Heavy-Ion Physics}




\section{II.C.1. Overview of the PHENIX Project PHENIX Collaboration}

PHENIX consists currently of over 350 scientists from 44 institutions in 10 countries. If you really would like to see a list of PHENIX collaborators, please contact Soren Sorensen. UT is within PHENIX mainly collaborating with the following institutions: ORNL, LANL, BNL, LLNL, Vanderbilt, Georgia State, Louisianna State, Columbia and Stony Brook in USA, BARC in India and finally IHEP in China.

Physics Goals of PHENIX. The primary goals of the PHENIX experiment at the Relativistic Heavy Ion Collider (RHIC) are to detect a new phase of matter, the quark-gluon plasma (QGP), and to measure its properties. During the past few years, many experimental probes of the QGP have been suggested. The PHENIX experiment measures many of the best potential signatures to see if any or all of these physics variables show simultaneous anomalies due to the formation of the QGP.

Table II.C.1.1 summarizes the physics quantities PHENIX measures and their relationship to the collaborations physics objectives. If the QGP phase transition occurs, one of the main goals for PHENIX is to characterize its nature. Two basic properties of the plasma, Debye screening of quantum chromodynamics (QCD) interactions and chiral symmetry restoration, are significant elements of our measurements. The thermal radiation of a hot gas is also extremely important; it characterizes the temperature of the system formed in these collisions. The strategy behind PHENIX is to study both lepton and hadron signatures in the same experiment. The leptons arise from the plasma phase and probe it directly, whereas the more copious hadrons provide the complementary information about the hadronization phase transition.

In order to study the physics quantities listed in Table II.C.1.1, electron pairs, muon pairs, photons, and charged hadrons must be measured. Electron and muon pairs are measured to study properties of the vector mesons (e.g., mass, width, yield), and to study continuum spectra in different regions of rapidity and mass. The $\mathrm{e} \mu$ coincidence probes charm production, and aids in understanding the shape of the continuum dilepton spectrum. Photon measurements probe the initial phase via prompt photons. Neutral mesons, such as $\pi^{0}$ and $\eta$, are also measured through the photon channel. Detection and identification of charged hadrons allow study of the $p_{T}$ distributions, production of antinuclei, the $\phi$ meson (via $\mathrm{K}^{+} \mathrm{K}^{-}$decay), jets, and Hanbury-Brown-Twiss (HBT) correlations. The special emphasis of PHENIX is to probe the proper- 


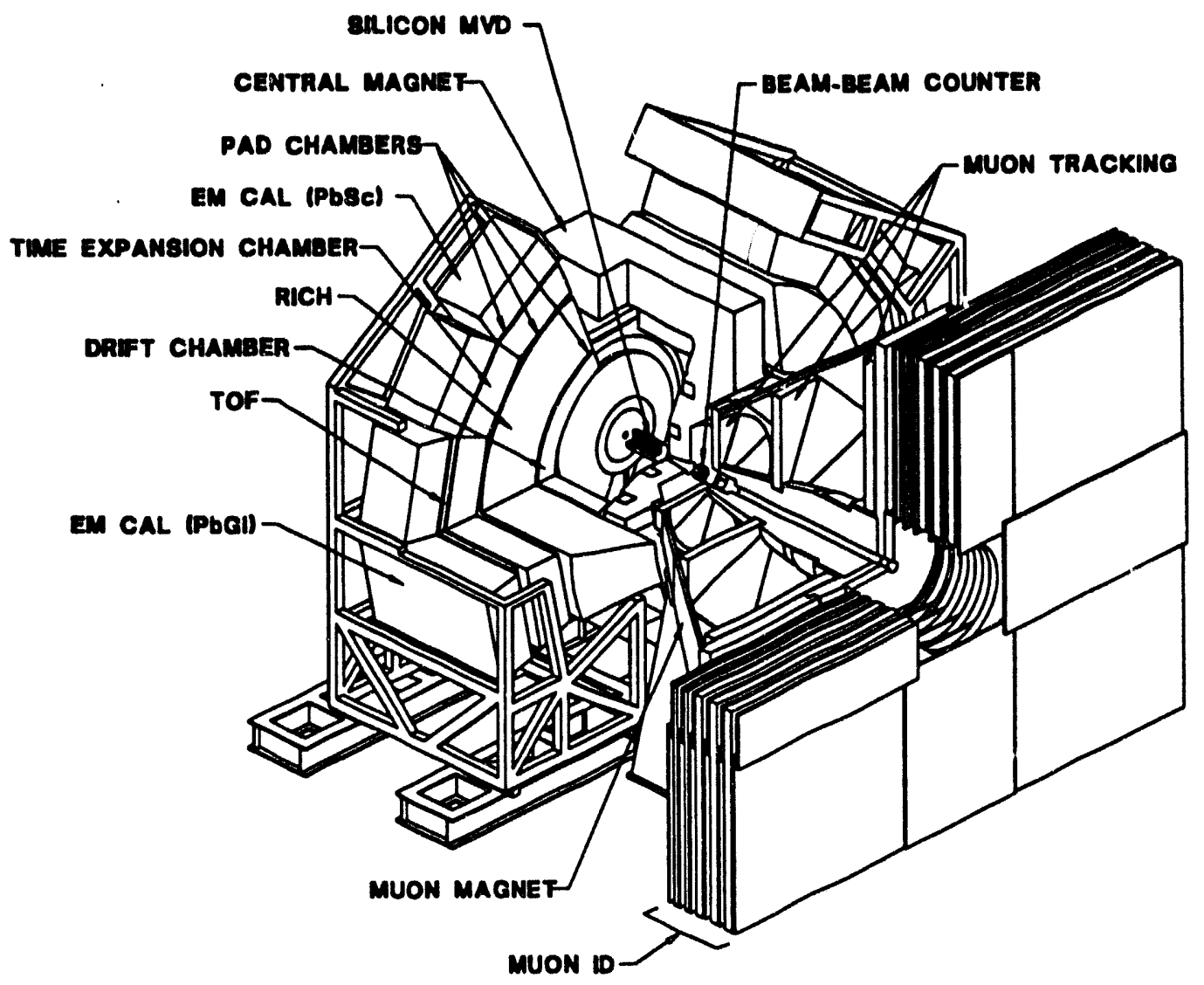

Figure II.C.1.1: A three-dimensional cutaway view of the PHENIX detector, showing the location of the various detector subsystems. The detector subsystems are labeled in the figure.

ties of matter from simultaneous measurements of these particles. Therefore, particle identification is an important element of the PHENIX experiment.

The PHENIX collaboration will search for the QGP through a programmatic study of a broad array of potential signatures, as a function of energy density in both $\mathrm{A}+\mathrm{A}$ and $\mathrm{p}+\mathrm{A}$ collisions. Recognizing that some of the potential signatures involve rare processes and small effects, PHENIX is designed to be a detector capable of taking data at the highest luminosities expected at RHIC. 
Table II.C.1.1: Physics Issues Related to Quark-Gluon Plasma

\begin{tabular}{|c|c|}
\hline QGP Physics Issues & Probes \\
\hline 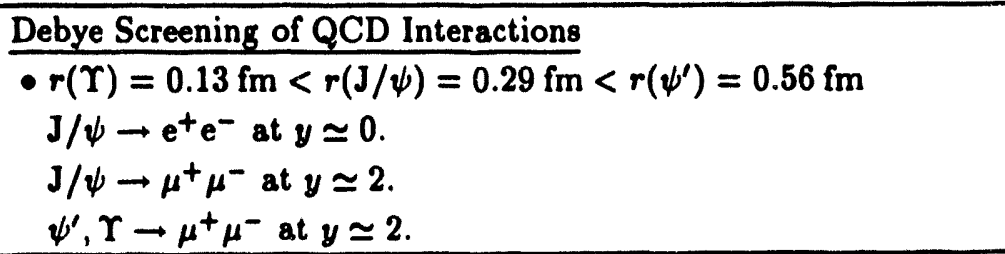 & Electrons \\
\hline 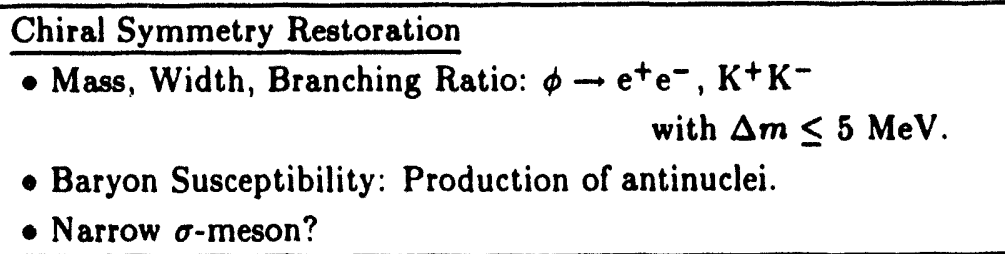 & $\begin{array}{l}\text { Electrons } \\
\text { Hadrons }\end{array}$ \\
\hline $\begin{array}{l}\text { Thermal Radiation of Hot Gas } \\
\text { - Prompt } \gamma, \text { Prompt } \gamma^{*} \rightarrow \mathrm{e}^{+} \mathrm{e}^{-} \text {. }\end{array}$ & Photons, Electrons \\
\hline $\begin{array}{l}\text { Deconfinement: Nature of the Phase Transition } \\
\text { - First-order: Entropy Jump } \rightarrow \text { Second rise in the }\left\langle p_{T}>\right. \\
\text { spectra of } \pi, \mathrm{K}, \mathrm{p} . \\
\text { - Second-order: Fluctuation } \rightarrow N\left(\pi^{0}\right) / N\left(\pi^{+}+\pi^{-}\right), d^{2} N / d \eta d \phi .\end{array}$ & $\begin{array}{l}\text { Hadrons } \\
\text { Hadrons, Photons }\end{array}$ \\
\hline $\begin{array}{l}\text { Strangeness and Charm Production } \\
\text { - Production of } \mathrm{K}^{+}, \mathrm{K}^{-}, \mathrm{K}_{\mathrm{L}}^{0} \\
\phi \rightarrow \mathrm{e}^{+} \mathrm{e}^{-}, \mathrm{K}^{+} \mathrm{K}^{-} \text {at } y \simeq 0 \\
\phi \rightarrow \mu^{+} \mu^{-} \text {at } y \simeq 2 \\
\text { D-meson: } \mathrm{e} \mu \text { coincidence. }\end{array}$ & $\begin{array}{l}\text { Hadrons } \\
\text { Electrons } \\
\text { Muons }\end{array}$ \\
\hline $\begin{array}{l}\text { Jet Quenching } \\
\text { - High } p_{T} \text { jets via leading particle spectra. }\end{array}$ & Hadrons \\
\hline$\frac{\text { Space-Time Evolution }}{\text { - HBT correlations for } \pi \pi \text { and KK. }}$ & Hadrons \\
\hline
\end{tabular}

Basic Outline of the PHENIX Detector. In order to have uniform $p_{T}$ acceptance for dielectrons, an axial field magnet was chosen with two electron detectors separated by $45^{\circ}$ in azimuth $(\phi)$. Electrons and positrons are detected by this two arm spectrometer, each arm subtending $90^{\circ}$ in $(\phi)$ and \pm 0.35 units in pseudorapidity $(\eta)$, roughly equivalent to a polar angle $(\theta)$ acceptance of $\pm 20^{\circ}$ centered at $\theta$ equal to $90^{\circ}$. The solid angle of each arm is 0.94 sr. Electron identification is performed using the following detector subsystems: Cherenkov radiation detected in the ring imaging Cherenkov counters 
Table II.C.1.2: Summary of the PHENIX Detector Subsystems.

\begin{tabular}{|c|c|c|c|}
\hline Element & $\Delta \eta$ & $\overline{\Delta \phi}$ & Purpose and Special Features \\
\hline Magnet: central (CM) & \pm 0.5 & $360^{\circ}$ & Up to $1.0 \mathrm{~T} \cdot \mathrm{m}$ \\
\hline muon (MM) & $1.1-2.5$ & $360^{\circ}$ & $0.72 \mathrm{~T} \cdot \mathrm{m}$ for $\eta=2,0.36 \mathrm{~T} \cdot \mathrm{m}$ for $\eta=1.3$ \\
\hline Silicon (MVD) & \pm 2.7 & $360^{\circ}$ & $d^{2} N / d \eta d \phi$, precise vertex. \\
\hline Beam-beam (BB) & $\pm(3.1-4)$ & $360^{\circ}$ & Start timing and fast vertex. \\
\hline Drift chambers (DC) & \pm 0.35 & $90^{\circ}+90^{\circ}$ & $\begin{array}{l}\text { Tracking, and good momentum and } \\
\text { mass resolution, } \\
\Delta m / m=0.4 \% \text { at } m=1 \mathrm{GeV} \text {. }\end{array}$ \\
\hline Pad chambers (PC) & & $90^{\circ}+90^{\circ}$ & $\begin{array}{l}\text { Pattern recognition and tracking } \\
\text { for nonbend direction. }\end{array}$ \\
\hline TEC & \pm 0.35 & $90^{\circ}+90^{\circ}$ & $\begin{array}{l}\text { Pattern recognition, tracking after } \\
\mathrm{RICH} \text {, and } d E / d x \text {. }\end{array}$ \\
\hline RICH & \pm 0.35 & $90^{\circ}+90^{\circ}$ & Electron identification. \\
\hline TOF & \pm 0.35 & $30^{\circ}$ & Good hadron identification, $\sigma<100 \mathrm{ps}$. \\
\hline $\mathrm{PbSc}$ & \pm 0.35 & $90^{\circ}+45^{\circ}$ & $\begin{array}{l}\text { Good e/ } \pi \text { separation at } p>1 \mathrm{GeV} / \mathrm{c} \text { by } \\
\mathrm{EM} \text { shower and } p<0.35 \mathrm{GeV} / \mathrm{c} \text { by } \\
\text { time-of-flight. } \\
\mathrm{K} / \mathrm{e} \text { separation at } 1 \mathrm{GeV} / \mathrm{c} \text { by } \\
\text { time-of-flight for } d E / d x \text {. }\end{array}$ \\
\hline $\mathrm{PbGl}$ & \pm 0.35 & $45^{\circ}$ & Photon detection. \\
\hline Muon chambers $(\mu \mathrm{T})$ & $1.15-2.35$ & $360^{\circ}$ & Tracking for muons. \\
\hline Muon identifier ( $\mu \mathrm{ID})$ & $1.15-2.35$ & $360^{\circ}$ & $\begin{array}{l}\text { Steel absorbers and chambers for } \\
\mu / \text { hadron separation. }\end{array}$ \\
\hline
\end{tabular}

( $\mathrm{RICH}$ ), energy loss of the charged particles in the time expansion chambers (TEC), and time-of-flight and electromagnetic showers in lead-scintillator $(\mathrm{PbSc})$ calorimeters. Within the aperture for the electron measurement are detector subsystems with limited acceptance for photons, using a lead-glass ( $\mathrm{PbGl})$ calorimeter $\left(\Delta \phi=45^{\circ}, \Delta \eta= \pm 0.35\right)$, and for charged hadrons, using a high precision time-of-flight (TOF) array $\left(\Delta \phi=30^{\circ}, \Delta \eta= \pm 0.35\right)$. The $\mathrm{PbSc}$ also measures photons with an energy resolution close to that achieved in the $\mathrm{PbGl}$; together, they comprise the PHENIX electromagnetic calorimeter (EMCal). A forward spectrometer $\left(10^{\circ}<\theta<35^{\circ}\right)$ around the beam axis is used to track and identify muons.

Figure II.C.1.1 is a three-dimensional cutaway view of the PHENIX de- 
tector, showing the location of the various detector subsystems. The detector subsystems are labeled in the figure. The acceptance and function of the detector subsystems are summarized in Table II.C.1.2.

\section{II.C.2. Muon Detection in PHENIX}

PHENIX Collaboration

Since 1987 the Nuclear Physics group at UT has been actively involved in preparations for a muon detector at RHIC. Originally we worked within the dimuon collaboration, headed by Glenn Young at ORNL. Two years ago this collaboration was fused togeiher with two other RHIC collaborations to form the current PHENIX collaboration. Our main reason for being so heavily involved with muon detection is based on the encouraging results from the NA38 collaboration at CERN on $J / \psi$ suppression in central heavy ion collisions. Currently we find this the most promising signature for the QuarkGluon Plasma. Since we focused on global observables in the first generation of high energy heavy ion experiments at CERN, we decided to go for a much more penetrating probe in the next generation of experiments.

Physics addressed by PHENIX's Muon Detector The dimuon measurement complements the dielectron measurement. In the light mass region, the muon arm measures $\rho$ and $\phi$ mesons with good efficiency, as well as the continuum $\left(1<m_{\mu^{+} \mu^{-}}<3 \mathrm{GeV}\right)$ at forward rapidities, thereby augmenting the significance of the midrapidity $\mathrm{e}^{+} \mathrm{e}^{-}$measurements.

Muon detection also allows a systematic study of the Debye screening effect on vector mesons from the measurements of $\mathrm{J} / \psi, \psi^{\prime}$ and $\Upsilon$, due to the different radii of these mesons: $R_{\Upsilon}(=0.13 \mathrm{fm})<R_{J / \psi}(=0.29 \mathrm{fm})<$ $R_{\psi^{\prime}}(=0.56 \mathrm{fm})$. As the energy density increases, $\psi^{\prime}$ should be suppressed first, followed by the $\mathrm{J} / \psi$ and the $\Upsilon$. The $\Upsilon$ serves as a calibration point in the measurement of the screening length, since its radius is much smaller than any estimated plasma screening radius at expected temperatures, meaning it may well never be suppressed at plasma temperatures attainable at RHIC.

The the Debye screening effects should disappear at sufficiently high $p_{T}$, while hadronic absorption effects should be similar for the $\mathrm{J} / \psi$ and $\psi^{\prime}$, and should decrease only slowly with $p_{T}$. Therefore, comparison of the $p_{T}$ dependence of the $\mathrm{J} / \psi$ to that of the $\psi^{\prime}$ will be essential to distinguishing between hadronic and plasma scenarios. The large acceptance of the muon arm provides sufficient counting statistics up to at least a $p_{T}$ of $5 \mathrm{GeV} / \mathrm{c}$. 
The high mass continuum $\left(m_{\mu \mu}>4 \mathrm{GeV}\right)$ is expected to arise primarily from hard-scattering (Drell-Yan). A measurement of this cross section is important, providing a reference point for studies of thermal pair emission at lower masses. Fixed-target experiments at FNAL, such as E772, show no dependence of this cross section on target mass beyond a linear scaling with the target mass, unlike the dependence observed for vector mesons and lowermass continuuri. However, such experiments also show significant variations in structure functions for different mass targets when considered as a function of $x_{1}, x_{2}$ or $x_{F}$. It is crucial to investigate these dependencies at RHIC energies in order to understand the QCD background to the thermal emission.

Copious production of open charm could populate the lepton pair spectrum in the 2-4 GeV mass region via semi-leptonic D-decays [1]. The contribution to the $\mathrm{e}^{+} \mathrm{e}^{-}$or $\mu^{+} \mu^{-}$mass spectrum from charm production is, thus, substantial. Preliminary results from E789 [2] indicate that the continuum below the $\mathrm{J} / \psi$ mass at approximately $x_{F}$ equal to zero is dominated by semileptonic $\mathrm{D} \overline{\mathrm{D}}$ decays. Clearly, it is essential to understand the charm contribution quantitatively. Furthermore, thermal charm production is interesting in itself since this production might carry direct information on the critical phase transition temperature [4].

It is difficult to separate charm contributions from the mass spectrum using muons or electrons alone. Unambiguous measurements of the $\mathrm{D} \overline{\mathrm{D}}$ cross sections can be made through the correlation of opposite sign e $\mu$ pairs, using the dimuon endcap in conjunction with the central electron spectrometer. The first measurement of the charm cross section in hadronic interactions was performed at the CERN ISR using two electron spectrometers with a dimuon spectrometer at $90^{\circ}$ between them [3]. None of the correlated e $\mu$ pairs can arise from either the Drell-Yan mechanism or thermal production. Like-sign $\mathrm{e} \mu$ pairs were, thus, used to remove the backgrounds due to random semileptonic decays of pions and kaons, thus providing the $\mathrm{D} \overline{\mathrm{D}}$ cross section near the region of its peak at $\eta=0$.

The PHENIX experiment, with its high-sensitivity electron and muon arms, is ideally suited to such a study. We plan to use electrons of $p_{T}$ greater than $1 \mathrm{GeV} / \mathrm{c}$ in the central region and muons with momentum greater than $2 \mathrm{GeV} / \mathrm{c}$ in the endcap muon arm. These cutoffs help avoid the large background rates of electrons due to Dalitz decays and photon conversions and of muons due to weak decays of pions and kaons. Even with these cutoffs, it is possible to measure pairs in the relevant mass range $\left(1.5<m_{e \mu}<6-7 \mathrm{GeV}\right)$ and at rapidities near zero. Note that in $p+p$ collisions, direct single $\mathrm{e}^{ \pm}$ 
production at a level $\mathrm{e} / \pi \approx 10^{-4}$ for $p_{T} \simeq 1 \mathrm{GeV} / \mathrm{c}$ is observed and is dominated by charm [5]. Once the measurements of $D \bar{D}$ cross sections have been measured in A + A collisions, one will be able to calculate and remove the charm contribution to the $\mathrm{e}^{+} \mathrm{e}^{-}$or $\mu^{+} \mu^{-}$mass spectrum, which is large in the region of $m_{l+l-}$ less than $4 \mathrm{GeV} / c^{2}$.

Overview of the PHENIX Muon Arm Detector. The PHENIX muon arm is designed to give PHENIX acceptance for particles decaying into lepton pairs emitted at rapidities away from $y=0$. The angular distribution of finalstate particles increases rapidly away from $y=0$, increasing as approximately $1 / \sin ^{2}(\theta)$ for a flat rapidity distribution. Detecting $\mathrm{e}^{+} \mathrm{e}^{-}$pairs becomes difficult in this environment, whereas detecting $\mu^{+} \mu^{-}$pairs remains technically feasible, provided that the absorber material used to remove hadrons is thin enough to preserve sufficient mass resolution for the $\mu^{+} \mu^{-}$pairs. The mass resolution for the PHENIX muon arm is sufficient to resolve the $\phi$ from the $\rho+\omega$, the $\psi^{\prime}$ from the $\mathrm{J} / \psi$, and the $\Upsilon(2 S+3 S)$ from the $\Upsilon(1 S)$, as was mentioned in Chapter 3.

An elevation view of the PHENIX muon arm and its relation to the PHENIX central magnet and the RHIC major facility hall is shown in Fig. II.C.2.1.

The muon arm covers polar angles $10^{\circ} \leq \theta \leq 35^{\circ}$. The limits to polar angle coverage result from the size of the piston in the muon magnet at small polar angles and the need to clear the RICH focal plane array at large polar angles. The arm has nearly full azimuthal angle coverage. Charged particles entering the muon arm are tracked by three stations of multi-plane drift chambers. Each station has six planes and three stereo views. These chambers must deliver position resolution per station better than $150 \mu \mathrm{m}$. The third station is more than $4 \mathrm{~m}$ in radius, which led to subdivision of the tracking stations into octants in azimuth.

The design of the muon arm deliberately has the absorber sections as thin as possible consistent with keeping the tracking chamber occupancies low enough to perform pattern recognition and obtain acceptable position resolution. Such an absorber does not provide sufficient hadron rejection for the "muon" sample, so a final detector section provides the needed additional hadron rejection. This detector, called the muon identifier, consists of several large walls of steel absorber interleaved with layers of limited streamer tubes of the Iarocci type. The streamer tubes are instrumented with two sets of pickup pads to give low resolution tracking capability and provide signals corresponding to a useful geometrical segmentation to the first level muon 


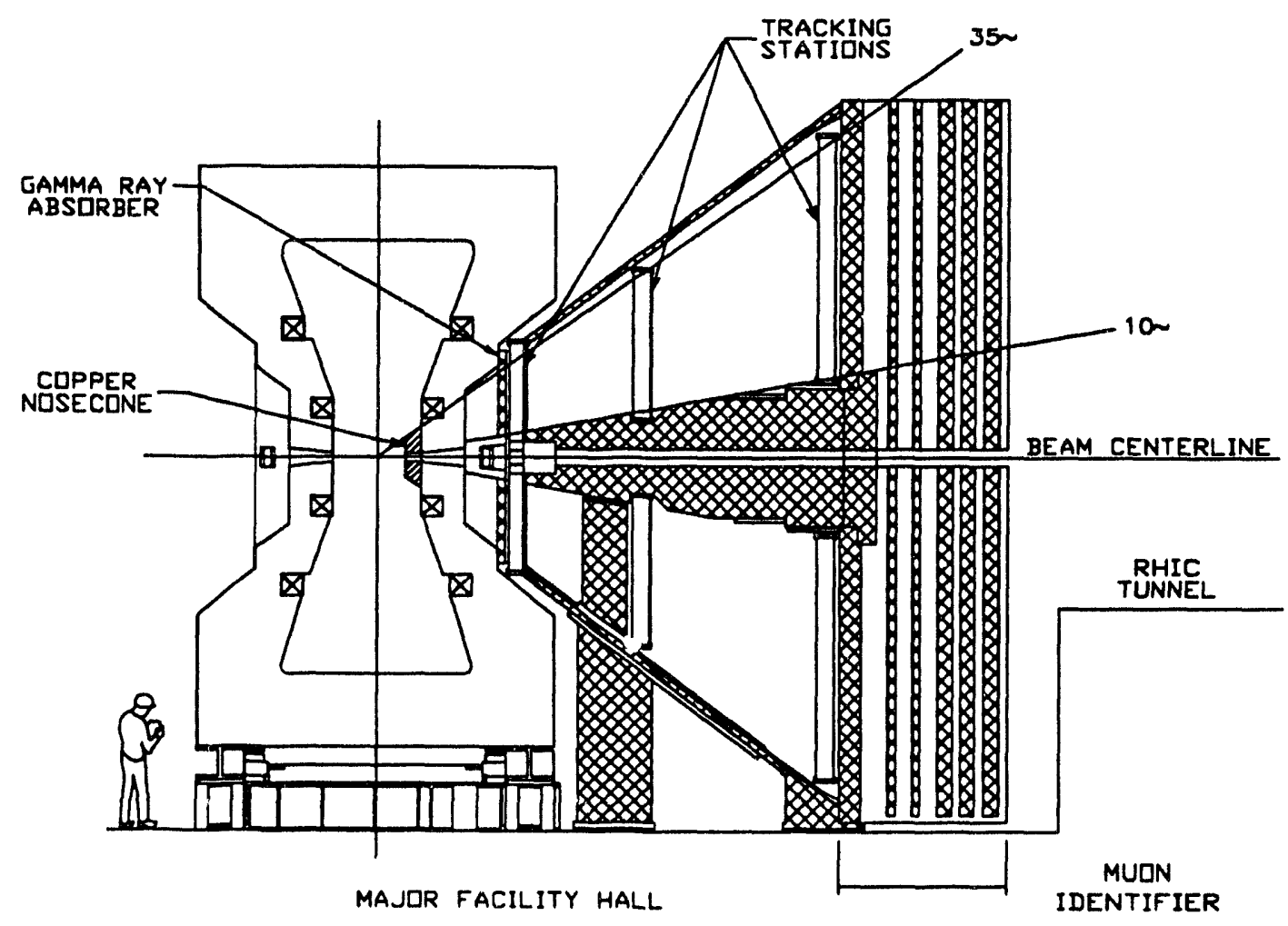

Figure II.C.2.1: Elevation view of PHENIX muon arm in the major facility hall.

trigger. The first absorber wall is the steel endplate flux return of the muon magnet.

Simulations of acceptance. The main purpose of the muon detectior is to detect muon pairs originating from vector meson decay or virtual photon production. This section will focus on the detector performance for simple events consisting only of a correlated pair of oppositely charged muons. All dimuon events have been generated with event generators based on Vogt's parameterizations [6] of the production cross sections for the vector meson resonances in heavy ion reactions, and their rapidity and $p_{T}$ distributions. The results presented assume a muon energy threshold of $2 \mathrm{GeV}$, which corresponds to a muon energy sufficient to penetrate to the deepest layer of the muon identifier. A lower muon threshold would be attained by including muons which penetrate through the second absorber layer only. 


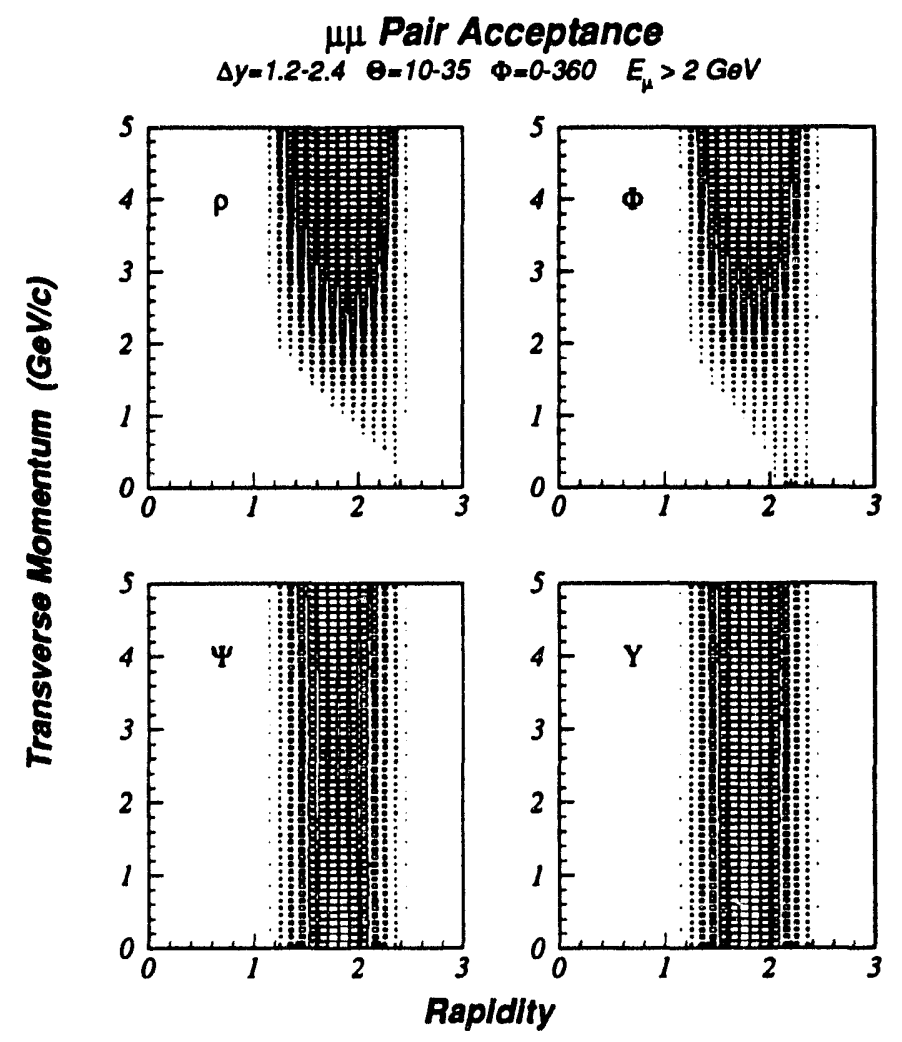

Figure II.C.2.2: Geometrical acceptance in rapidity versus transverse momentum for the $\rho, \phi, \mathrm{J} / \psi$, and $\Upsilon$.

The geometrical acceptance, assuming uniform distributions in rapidity and $p_{T}$, for the $\rho, \phi, \mathrm{J} / \psi$, and $\Upsilon$ is shown in Fig. II.C.2.2. The effect of the 2 $\mathrm{GeV}$ muon threshold is seen as a cutoff at low transverse momentum for the lighter mass vector mesons. The cutoff appears at a transverse mass of around $2-3 \mathrm{GeV} / \mathrm{c}^{2}$. The transverse momentum cutoff decreases at forward rapidity due to the boost received.

The total acceptance, integrated over the expected rapidity and transverse momentum distributions is $0.13 \%, 0.38 \%, 5.4 \%$, and $5.9 \%$ for the $\rho, \phi$, $\mathrm{J} / \psi$, and $\Upsilon$, respectively. The integrated acceptance decreases rapidly with decreasing mass due to the transverse mass cutoff resulting from the momentum threshold of the spectrometer (see Fig. II.C.2.2).

The advantages of detecting dileptons to obtain information on the hot and dense phase of high energy nuclear collisions have been extensively discussed. The disadvantage of using non-strongly interacting probes is the much smaller cross sections and yields. In the following, we will address the crucial 
High Energy Heavy-lon Physics

question of the expected yields of the vector mesons in the muon detector. It is important to note that the large acceptance of the muon arm provides a significantly larger rate than the electron measurement for higher mass dileptons, and the two measurements are complementary in mass as well as rapidity range.

All cross section estimates are based on the observation that hadroproduction of vector mesons in nucleon-nucleon collisions can be expressed as $([6,7])$

$$
\begin{aligned}
B_{R} \sigma & =A_{R} e^{-14.7 \tau}, \\
\tau & =\frac{M_{R}}{\sqrt{s}} .
\end{aligned}
$$

Here $M_{R}$ is the resonance mass, $B_{R}$ is the branching ratio of the vector meson decay to $\mu^{+}+\mu^{-}$and $A_{R}$ is the normalization coefficient. The original parameterization in Ref. [7] was given in terms of $d \sigma /\left.d y\right|_{y=0}$, whereas we have chosen to use the integrated cross section. Further details on the rapidity- and $p_{T}$-distributions of vector meson production can be found in Ref. [6]. Values of $A_{R}$ for the six most important vector mesons can be found in Table II.C.2.1. The $B_{R} \sigma$ values for minimum bias heavy ion reactions between identical nuclei $\mathrm{A}+\mathrm{A}$ have been obtained from the nucleon-nucleon values by scaling with $A^{2 \alpha}$, where $\alpha_{\rho, \phi}=0.85, \alpha_{J / \psi, \psi^{\prime}}=0.90$ and $\alpha_{\Upsilon, \Upsilon^{\prime}}=0.95$. For central collisions the cross sections have been assumed to scale with $A^{4 / 3 \alpha}$, where the factor $4 / 3$ in the exponent describes the scaling of the number of binary nucleon-nucleon collisions. This scaling for central collisions agrees very well with Gerschel and Hufner's parameterization of $\mathrm{J} / \psi$ cross sections in nucleus-nucleus collisions at SPS energies [8].

Based on the cross sections given in Table II.C.2.1 and Ref. [6], the number of accepted dimuon events per RHIC year (assumed to be 3000 hours at a nominal luminosity of $2 \times 10^{27}$ for $\mathrm{Au}+\mathrm{Au}$ and $1.5 \times 10^{29}$ for $\mathrm{p}+\mathrm{Au}$ ) are calculated and shown in Table 3.3. Note the large yields obtained with the muon detector. The nearly two million accepted $\mathrm{J} / \psi$ events in a nominal RHIC year allow a detailed subdivision of the data set to study impact parameter, rapidity, and $p_{T}$ dependencies. The errors quoted for central $\mathrm{Au}+\mathrm{Au}$ events in Table 3.4 have been obtained by estimating the statistical errors involved in the subtraction of the combinatorial background below the resonances. It is noteworthy, that even though the $\rho$ and $\phi$ resonances have substantial background their yield can nevertheless be estimated to better than $10 \%$. This will make the muon detector the primary tool in PHENIX for studying the $\rho$. 
Table II.C.2.1: Cross sections for production of vector mesons decaying into the dimuon exit channel in $100+100 \mathrm{GeV} / \mathrm{c} \mathrm{Au}+\mathrm{Au}$ collisions. $A_{R}$ and $B_{R}$ are defined in the main text. $P_{e}$ is the production probability per event. Central events are defined as the most central $1 \%$ of the reaction cross section.

\begin{tabular}{|c|c|cc|cc|}
\hline \hline & & \multicolumn{2}{|c|}{ Minimum Bias Au $+\mathrm{Au}$} & \multicolumn{2}{|c|}{ Central Au+Au } \\
\hline & $\begin{array}{c}A_{R} \\
\left(\mathrm{~cm}^{2}\right)\end{array}$ & $\begin{array}{c}B_{R} \sigma \\
\left(\mathrm{cm}^{2}\right)\end{array}$ & $P_{e}$ & $\begin{array}{c}B_{R} \sigma \\
\left(\mathrm{cm}^{2}\right)\end{array}$ & $P_{e}$ \\
\hline$\rho$ & $6.07 \times 10^{-30}$ & $3.96 \times 10^{-26}$ & $6.46 \times 10^{-3}$ & $4.92 \times 10^{-27}$ & $8.02 \times 10^{-2}$ \\
$\phi$ & $9.34 \times 10^{-31}$ & $6.09 \times 10^{-27}$ & $9.94 \times 10^{-4}$ & $7.56 \times 10^{-28}$ & $1.23 \times 10^{-2}$ \\
$\mathrm{~J} / \psi$ & $1.43 \times 10^{-31}$ & $1.48 \times 10^{-27}$ & $2.42 \times 10^{-4}$ & $1.63 \times 10^{-28}$ & $2.66 \times 10^{-3}$ \\
$\psi^{\prime}$ & $2.15 \times 10^{-33}$ & $2.00 \times 10^{-29}$ & $3.26 \times 10^{-6}$ & $2.29 \times 10^{-30}$ & $3.74 \times 10^{-5}$ \\
$\Upsilon$ & $3.08 \times 10^{-34}$ & $3.80 \times 10^{-30}$ & $6.20 \times 10^{-7}$ & $4.84 \times 10^{-31}$ & $7.90 \times 10^{-6}$ \\
$\Upsilon^{\prime}$ & $3.08 \times 10^{-35}$ & $3.80 \times 10^{-31}$ & $6.20 \times 10^{-8}$ & $4.84 \times 10^{-32}$ & $7.90 \times 10^{-7}$ \\
\hline \hline
\end{tabular}

Track Reconstruction Performance Calculations The following discussion of the simulations of the performance of the muon arm spectrometer is organized by increasing hit multiplicity. First, single track issues such as momentum resolution are discussed, followed by double track characteristics such as dimuon invariant mass resolution and acceptance estimates. The majority of these calculations were done by UT.

The tracking procedure for the spectrometer consists of two steps: (1) finding the track segments from the hits in each station, and (2) joining the track segments from the three stations.

The track reconstruction uses simple tree searches for correlated hit candidates combined with a Principal Component Analysis (PCA) test [9] to select the correct candidates. Let us assume a single track generates a $\mathrm{M}$ dimensional hit vector $\vec{h}=\left(h_{1}, h_{2}, . ., h_{i}, . ., h_{M}\right)$. For step (1) the hits are the hit positions in each of the six drift chambers in the $U U^{\prime} Y Y^{\prime} V V^{\prime}$ geometry. For step (2) the first four coordinates in the hit vector $\left(h_{1}, h_{2}, h_{3}, h_{4}\right)$ will be the $\theta$ and $\phi$ of the track segment position and the $\theta$ and $\phi$ of the track segment local direction vector within the first tracking station. The next four coordinates will be the same track segment information from the second tracking station.

The algorithm is tuned using "learning tracks"; from PISA simulations 
$N$ learning tracks $\vec{h}_{n}$ are generated $(N>1000)$ and the following average hit vector $\vec{H}$ and dispersion matrix $\mathbf{A}$ are created

$$
\begin{aligned}
H_{i} & =\frac{1}{N} \sum_{n=1}^{N}\left(h_{i}\right)_{n} \\
A_{i j} & =\frac{1}{N} \sum_{n=1}^{N}\left[\left(h_{i}\right)_{n}-H_{i}\right]\left[\left(h_{j}\right)_{n}-H_{j}\right]
\end{aligned}
$$

It is important to note that at a more advanced stage of the experiment the $N$ learning tracks should not be taken from simulations, but instead from low multiplicity calibration data.

Finally the $M$ eigenvalues $\lambda_{i}$ and $M$ eigenvectors $\vec{\omega}_{i}$ of the dispersion matrix $\mathbf{A}$ are calculated. The eigenvalues can be organized as a vector $\vec{\lambda}$ and the eigenvectors can be organized into a $M \times M$ matrix $\omega$ with components $\omega_{i j}$.

Based on $\vec{\lambda}$ and $\omega$ it is now possible to use the following very sensitive criteria to test if a track candidate $\vec{c}=\left(c_{1}, c_{2}, . ., c_{i}, . ., c_{M}\right)$ constitutes a true track. The generalized distance, $d$, of $\vec{c}$ from an ideal track can be defined as

$$
\begin{aligned}
\eta_{j} & =\sum_{i=1}^{M} \omega_{i j}\left(c_{i}-H_{i}\right) \\
d^{2} & =\frac{1}{M} \sum_{j=1}^{M} \frac{\eta_{j}^{2}}{\lambda_{j}}
\end{aligned}
$$

$d$ will be approximately normally distributed with a standard deviation of 1 . If $d$ is not normally distributed then the learning data set contains abnormalities and should be carefully analyzed. If it is now required for a track candidate that $d<d_{\max }$, then only a small fraction of all real tracks will be rejected, whereas a very large fraction of spurious tracks will be rejected. For $d_{\max }=$ 3 only $0.3 \%$ of the real tracks are rejected. The fraction rejected depends on the relative magnitude of the eigenvalues. In a high resolution detector, the smallest eigenvalues become extremely small and the rejection power of the PCA test is very good. The PCA test constitutes the best possible rejection test based on a linear method; the linearity ensures that the PCA is a very fast rejection test.

The track segment finding within a tracking station proceeds according to the following scheme: 


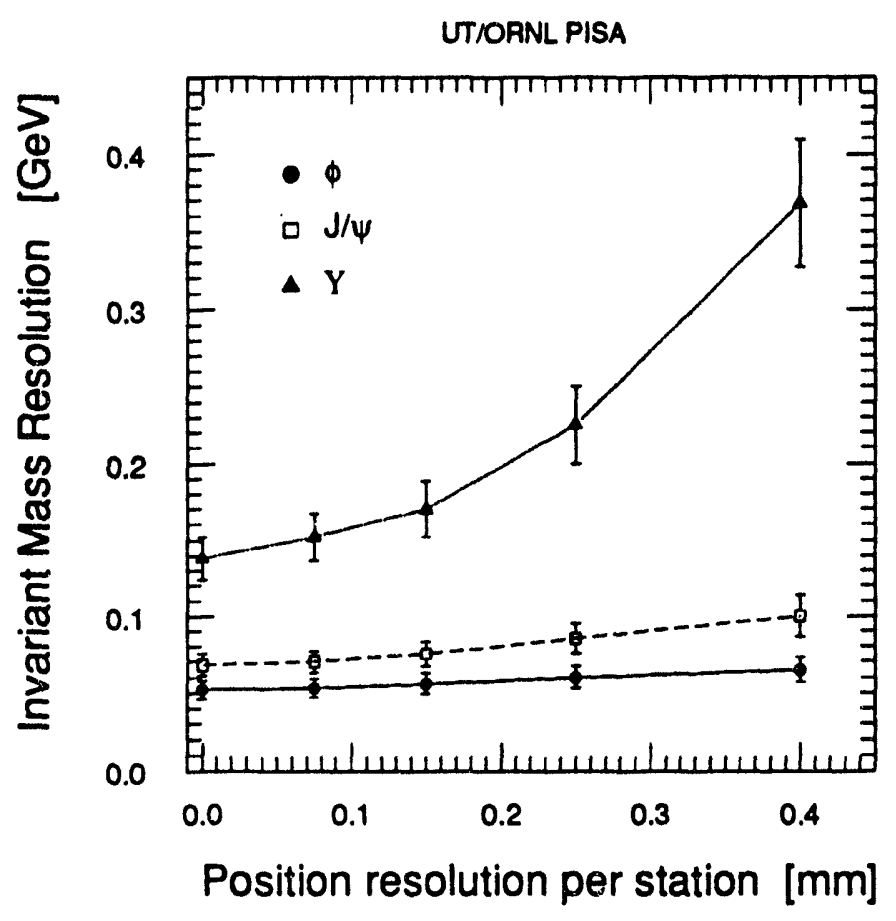

Figure II.C.2.3: Invariant mass resolution of dimuons originating from $\phi, \mathrm{J} / \psi$ and $\Upsilon$-decay as a function of the position resolution in the spectrometer tracking stations.

1. First, all possible $U U^{\prime}, Y Y^{\prime}$ and $V V^{\prime}$ hit pairs are identified by searching for hit positions in the two planes smaller than $L_{p} \times$ the plane separation. where $L_{P}$ is typically $2-3$. The average position $\bar{h}_{k}$ for each of the possible pairs is calculated.

2. A PCA test with $d_{\max }=5$ is applied to all sets of 3 pairs, $\left(U U^{\prime}, Y Y^{\prime}\right.$ and $V V^{\prime}$. Due to the large value of $d_{\max }$ this first test keeps all real tracks, but rejects the obviously false cases and reduces the total number of track candidates substantially.

3. A more sensitive PCA test with $d_{\max }=4$ is now applied to the complete 6 -dimensional set of possible hits surviving step 2 .

4. The left-right ambiguities are resolved by calculating all $2^{6}$ possible real hit combinations to find which one gives the smallest PCA distance.

5. Finally, a global search is performed for the largest set of tracks which 
both minimizes the sum of the PCA distances and can be removed without incorporating the same hit in two tracks (special provision is, however, taken for unresolved double hits).

6. For each well-identified track segment the following 4 quantities are stored: $\left(\theta_{r}, \phi_{r}, \theta_{p}, \phi_{p}\right)$ where $\theta$ and $\phi$ are the polar and azimuthal angles and the indices $r$ and $p$ denote the position and momentum.

The joining of track segments between tracking stations into a complete track is actually somewhat simpler than finding the segments. The following procedure is used:

1. All possible hit combinations from station \#2 and \#3 are formed (typically $30 \times 40$ candidates in central collisions).

2. A PCA test with $d_{\max }=4$ is performed to identify reasonable track candidates. Typically 50-200 candidates survive.

3. All track candidates from 2) are combined with track segments from station \#1 and a PCA test with $d_{\max }=3$ is performed to select the final tracks. Since a total of $3 \times 4=12$ track segment coordinates enters in this calculation and each track is determined by only 5 parameters, the problem is highly overdetermined and the final PCA test identifies the true tracks extremely efficiently.

As stated earlier, the main purpose of the muon detector is to detect muon pairs originating from vector meson decay or virtual photon production. The most important two-track parameter is the invariant mass resolution, which should be as small as possible to provide a good signal-to-background ratio. The resolution of $M_{\text {inv }}$ below $4 \mathrm{GeV} / \mathrm{c}^{2}$ is dominated by the straggling in the absorber, whereas at higher masses the dominant contribution is the position resolution of the drift chambers. The asymmetric shape of the peaks, with a low energy tail, is caused by Landau fluctuations. The relative mass resolution is approximately given by $\sigma(M) / M=6 \% / \sqrt{M}$; this mass resolution enables a clear separation of $\rho$ and $\phi, \mathrm{J} / \psi$ and $\psi^{\prime}$, with an acceptable separation of $\Upsilon$ and $\Upsilon^{\prime}$.

To specify the drift chamber design criteria, the dependence of the $M_{\text {inv }}$ resolution as a function of the position resolution in a complete tracking station was investigated. The calculation shown in Fig. II.C.2.3 was performed with an ORNL/UT algorithm including a correction for straggling, so the absolute values of the $M_{\text {inv }}$ resolution differ slightly from the values above. The $\mathrm{J} / \psi$ 
mass resolution is nearly independent of the position resolution, since the dominant contribution here is the straggling in the absorber, whereas the $\Upsilon$ mass resolution varies strongly with the position resolution. Note that Fig. II.C.2.3 shows the position resolution of a complete tracking station, not the position resolution of each individual drift chamber. If the position resolution of each of the six individual drift chambers within a tracking station is conservatively assumed to be $0.3 \mathrm{~mm}$, the total tracking station position resolution would be $0.14 \mathrm{~mm}$.

\section{II.C.3. The Off-line Computing System of PHENIX PHENIX Collaboration}

Introduction. For the next five years one of our main responsibilities will be the design and implementation of the off-line computer system of PHENIX. Prof. Sorensen has together with Prof. Charlie Maguire from Vanderbilt University, Dr. Tom Carey from Los Alamos National Laboratory and Prof. Bill Zajc from Columbia University responsibility for all aspects of computing, data acquisition, online, off-line and simulations. In particular we will be concerned with the off-line system. In the following we will describe the main aspects of this system.

The purpose of the PHENIX off-line computing system will be to provide all aspects of data and information handling that are not directly connected to the collection of data and the monitoring of the experiment. Examples of functionalities provided by the off-line system are: event and detector simulation, event reconstruction and analysis, and data and general information management.

The off-line system can be viewed as another subdetector, which will have to undergo the usual engineering phases: design, prototyping, fabrication, implementation and maintenance. The off-line system does, however, function in a somewhat different way, because it also can be viewed as a service organization for the rest of the collaboration. It will have to provide all the necessary tools for the physicists to transform the data, originally written to a storage medium by the data acquisition system in some digital format, into interesting physics papers and exciting conference talks. Whereas only a small subset of physicists will be directly interacting with each of the subdetectors, practically all members of the collaboration will interact with the off-line system on a daily basis (e.g., analyzing data, reading online manuals, producing figures, or accessing information on the state of the detector during a run two years). 
Table II.C.3.1: Data Storage and CPU requirements for PHENIX and selected RHIC and SSC detectors.

\begin{tabular}{|l||r|r|r|r|}
\hline \hline & PHENIX & STAR & PHOBOS & SDC \\
\hline Event recording rate (Hz) & 100 & 1 & 400 & 100 \\
Event size (kBytes) & 300 & 20,000 & 12 & 1000 \\
Recording rate (MBytes/s) & 20 & 20 & 5 & 100 \\
\hline Events / year (10 $)$ & 650 & 10 & 4000 & 1000 \\
Raw data / year (10 & 200 & 200 & 50 & 1000 \\
\hline Gigaflops-sec / eveni & 1.0 & 10 & 0.005 & 0.3 \\
Continuous CPU need (Gflops) & 65 & 10 & 2 & 30 \\
\hline \hline
\end{tabular}

Requirements. The initial phase in the design of the PHENIX off-line system has been an evaluation of the physics and functional requirements. The two most important and expensive requirements by far are: (1) to have sufficient CPU power for the initial event reconstruction of the raw data, and (2) to be able to store all the recorded data (raw and simulated). Table II.C.3.1 summarizes the basic parameters for the estimation of these requirements for PHENIX, STAR and PHOBOS [10] and the SDC detector [11] from the former SSC. Both PHENIX and STAR expect a recording rate of $20 \mathrm{MBytes} / \mathrm{s}$.

The assumptions given above lead to an estimated 200 TeraBytes of raw data each year. It can be assumed, that additionally 300 TeraBytes of simulated data and reconstructed data will need to be stored resulting in a total storage need for PHENIX around 0.5 PetaBytes each year. This requirement is 2-3 orders of magnitude larger than current experiments and corresponds to $\approx 100,0008 \mathrm{~mm}$ videotapes. An additional requirement for the data storage system will be to store as much of the data as possible with access times less than a few minutes.

In order to estimate the total need for CPU power it is necessary to estimate the CPU consumption per event. With the current tracking algorithms in PISORP 2.2 a full reconstruction of a central Au+Au event takes approximately 1-2 minutes on a IBM RS6000 workstation, corresponding to 1000 Gigaflops-seconds. With an event rate of $65 \mathrm{~Hz}$ this implies a continuous $C P U$ need around 65 Gigaflops for event reconstruction. If the event reconstruction facility has an "uptime" of $90 \%$ the CPU need reduces to 40 Gigaflops, but this reduction is compensated by the additional need for production of sim- 


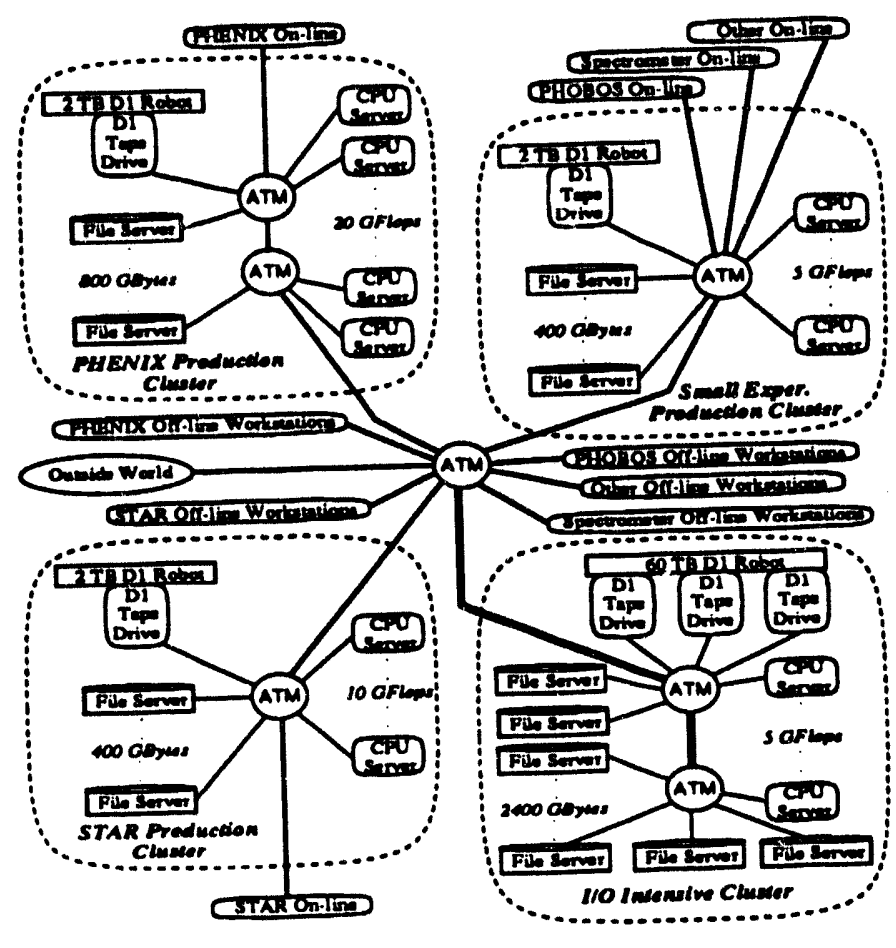

Figure II.C.3.1: "Strawman" model of the proposed RHIC Computing Facility. The center will contain 4 clusters. PHENIX will have its own cluster for event reconstruction and have shared access to the $\mathrm{I} / \mathrm{O}$ Intensive cluster for data analysis.

ulated data and repeated complicated data analysis. The magnitude of this CPU power requirement can be put in perspective by the fact, that the largest commercially available systems currently is around 10-20 Gigaflops.

The RHIC Computing Center. In order to meet the requirements outlined in the previous section a simple model for PHENIX's off-line computing system has been developed. A fundamental assumption within the model is, that the bulk of the computing will be done at a central RHIC computing facility (RHICCF), which will provide both hardware and manpower for a large fraction of PHENIX's needs. The current "strawman" model for RHICCF is shown in Fig. II.C.3.1 obtained from a report by the RHIC Off-line Computing Study Group chaired by B. Gibbard [12].

The model operates with 4 main clusters of computing facilities: production clusters for PHENIX, STAR, a common cluster for the small experiments, 
The Main Event Handling Module in The Orf-line and Simulation System:

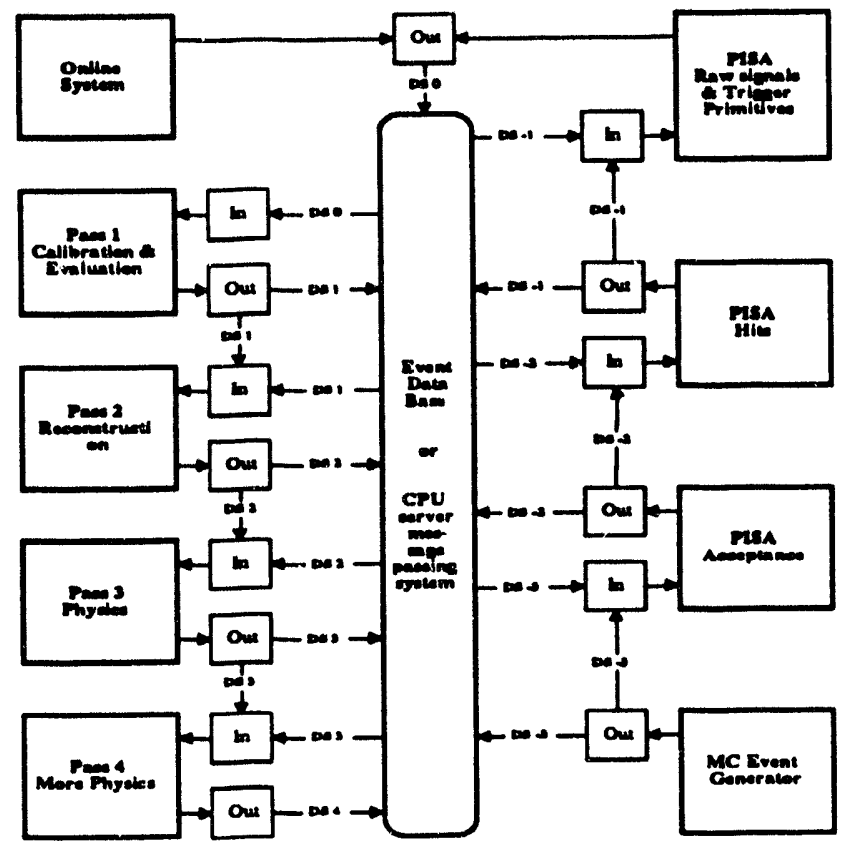

Figure II.C.3.2: Conceptual layout of the main modules of the event handling package and the simulation package. The data flow through the simulation system will be going from the event generator module at the lower right up to the digitization mocule in the upper right corner. The output from this moviuie and the on-line system will be accepted by the off-line system for calibration, event reconstruction and data analysis. The main modules will exchange information through a set of data structures (labeled DS). This design will provide a high degree of modularity.

and a common cluster for $\mathrm{I} / \mathrm{O}$ intensive tasks. Each production cluster will consist of a) a set of CPU servers scaled to the CPU needs of the particular experiment, b) a file server for short time storage of data and experiment specific databases, and c) a tape drive system with an intermediate size tape robot. The I/O intensive cluster, which will be shared between all RHIC users, will contain a large scale disk and tape storage system. It is envisioned, that the data reconstruction is taking place at the production cluster and the data analysis is done at the $\mathrm{I} / \mathrm{O}$ intensive cluster.

In addition to providing the bulk of the hardware needs for PHENIX's offline system RHICCF will also provide a substantial amount of manpower and 
software for core tasks like a) general system support, b) network support, c) code development support, d) general application (CERNLIB, GUI, databases, publishing etc.) e) technical development and f) hardware support. contain:

The current model assumes PHENIX's production cluster allocation to

CPU Servers:

File Servers:

Mass Storage Playback:

Mass Storage Robot:

Network:

In addition PHEI

which currently is envis.

CPU Servers:

File Servers:

Mass Storage Playback:

Mass Storage Robot:

Network:

Probably the most important task of the off-line system is to organize and provide access to the multitudes of data collected by the collaboration. The data can be subdivided into three different classes: a) Simulated or real event data, b) detector and instrumental data and c) general information data. This division of all the PHENIX data into 3 distinct classes is shown in Fig. II.C.3.3, which illustrates a conceptual layout of the general PHENIX multidatabase system.

\section{II.C.4. The RD93 $\mu$ ID Project}

E. C. Cornell ${ }^{6}$, E. J. Howell ${ }^{2}$, Xiaochun He${ }^{5}$, W. Kehoe' ${ }^{1}$, H. J. Kim ${ }^{4}$, P. Kirk ${ }^{3}$, J. G. $\mathrm{Kreke}^{5}$, X.T. Liu ${ }^{2}$, F. E. Obenshain ${ }^{4}$, G. A. Petitt' ${ }^{2}$, S. J. Sorensen ${ }^{5}$, G. R. Young ${ }^{4}$, Ying-Chao Wang $^{3}$, Zhi-Fu Wang ${ }^{3}$, Ziyang Zhang ${ }^{2}$ 
Conceptual Layout of PHENIX's Multi-database

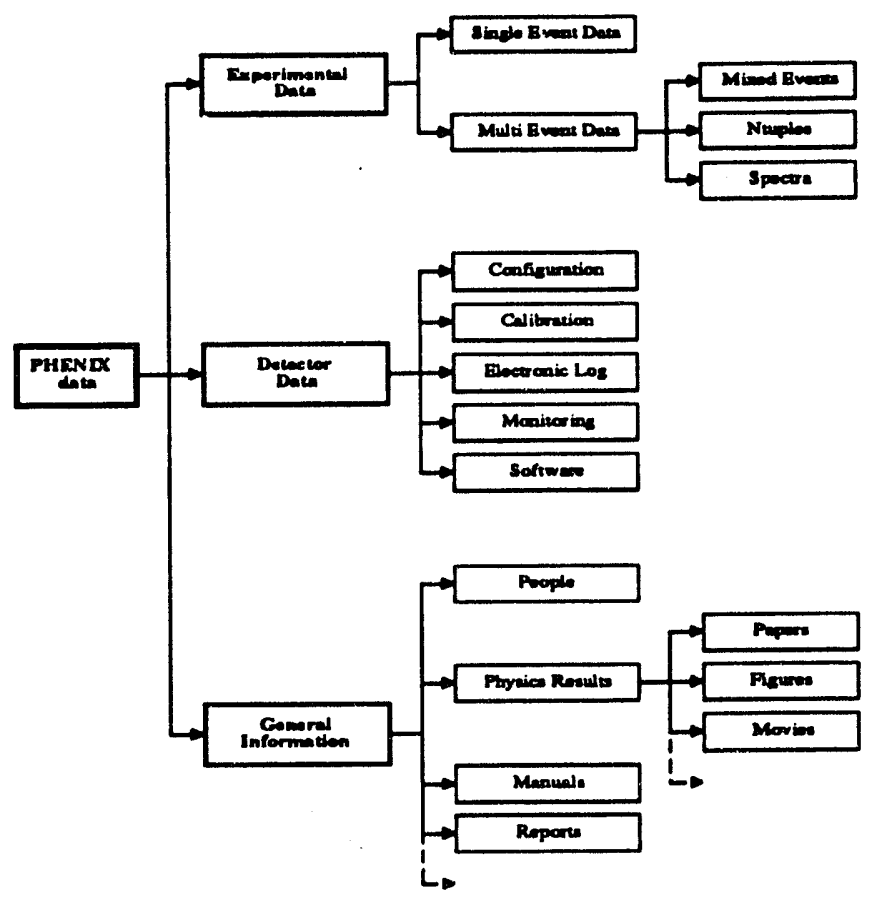

Figure II.C.3.3: Conceptual layout of PHENIX's multidatabase.

UT collaborated in a PHENIX R\&D project at the Brookhaven National Laboratory (BNL) Alternating Gradient Syncrotron (AGS) during the summer (1993) running of the proton beam. The purpose of the project, located on the B2 test beamline, was to study optimization of muon identification in a multiparticle environment, over a large geometrical acceptance, in the momentum range of $1.0-8.0 \mathrm{GeV} / \mathrm{c}$. The Muon Arm of the PHENIX detector expects to operate in such an environment. This project, colloquially termed the "RD93" experiment, is a continuation of the earlier RD10 and RD45 experiments, also located at the BNL AGS test beamline (then, $f_{2} 2$ ). All three experiments used sampling calorimeter technology to study leptonic and hadronic interactions, primarily considering shower volumes, penetration characteristics, interaction lengths, and leakage distributions. RD93, however, shifted emphasis away from leakage studies toward better particle identification, by concentrating on shower profiles and incident muon identification. RD93 replaced all the downstream leakage detectors of the earlier experiments with three sets of muon "tagging" arrays separated by two walls of thick $4 \lambda$ steel blocks. Also, RD93 redistributed its overly-instrumented calorimeter $i y$ more than doubling 
its lateral resolution while keeping the longitudinal segmentation to under $1 \lambda$. Over-instrumenting the calorimeter will aid in establishing design criteria for the minimum resolution needed for the Muon Identifier in PHENIX. In addition, results of the RD93 experiment, like those of the earlier experiments, can then benchmark various computer Monte Carlo routines (i.e. GEANT) which simulate detector response.

The RD93 experiment consisted of 690 particle detectors that fall into one of two categories: experimentation or beam definition. The experimental detectors are themselves not experimental but Limited Streamer Mode (Iorrocci) Tubes (LST) with either strip or pad readout and make up the active portion of the muon identifier. The identifier operated in two "modes": strip mode or pad mode with the pad mode requiring 36 more channels. The beam defining detectors consist of scintillating plastic and Cherenkov counters, all with photomultiplier (PMT) readout. Most of the beam definition occurs upstream of the identifier with 3 start counters, a veto counter, and 3 Cherenkov counters arranged in tandem, but for higher momentum incident particles $(>2 \mathrm{GeV} / \mathrm{c})$, a set of detectors behind the identifier, the $\mu$-taggers, aided in the discrimination of muons and pions.

The design of the RD93 $\mu$ ID was largely based on limiting the number of calorimeter cells to 10 , using an overall thickness to be approximately that used in earlier experiments for continuity, using steel for the absorber, making the calorimeter as thick in $\lambda$ as possible for best $\pi / \mu$ separation, and limiting the channel count to the available electronics. The earlier experiments used plastic scintillator with photomultiplier (PMT) readout for the active layers in the calorimeter. RD93, however, used Limited Streamer Mode Tubes (LSTs) with capacitively coupled readout strips or pads. LSTs seemed a viable instrument for PHENIX's $\mu$ ID because of their reliability, low cost, low maintenance and proven success; yet, their slow timing characteristics (approx. 110ns) virtually preclude their use as a second-level trigger, required in PHENIX. Nevertheless, timing was not an essential part of RD93 so valuable information can still be obtained.

The final design for the RD93 $\mu$ ID used 10 cells, each with one $4 \mathrm{ft} \times 4 \mathrm{ft}$ $\mathrm{x} 5.5$ in thick steel absorber followed by the active layer composed of twelve LSTs stacked in an array. Each array had 24 pick-up strips on either side, front and back, one side oriented horizontally, the other vertically fo: a total of 48 channels per active cell plane. Strip geometry defined spatial resolution with dimensions of $1 \mathrm{~m} \times 4 \mathrm{~cm}(\mathrm{x}, \mathrm{y})$; Pad size was approximately $22 \mathrm{~cm} \times 22 \mathrm{~cm}$. Combined spatial resolution in strip mode was then $4 \mathrm{~cm} \times 4 \mathrm{~cm}$ at each plane, 
barring hodoscopic ambiguity. Each array required a gap of $2.5 \mathrm{in}$ between absorber layers, yielding a cell thickness of $8.0 \mathrm{in}$ and an overall thickness of $80.0 \mathrm{in}$ with $55.0 \mathrm{in}(8.3 \lambda)$ of steel comparing closely to the twelve supercell steel calorimeter used in RD45. Hence, $\mu / \pi$ separation was $2.5 e-4$ behind the RD93 $\mu \mathrm{ID}, 4.6 \mathrm{e}-6$ behind the first steel wall and $8.3 \mathrm{e}-8$ behind the second steel wall. Ana'ysis of the RD93 experiment is underway and details appear in Sec. II.C.5.. Due to problems in the data acquisition, some runs have to be retaken this year in the follow-up, "RD94", experiment also at the B2 line at the AGS. Those runs along with their normalizing "minimum-bias" runs must be retaken. The data acquisition system otherwise worked quite well, with acquisition rates of approximately 25 events per second (see Sec. II.C.7.). To replace lost runs, increase statistics, and maintain continuity within a run set (a given momentum), the RD93 collaboration has requested rerunning all of RD93. Other than repairs, RD94 expects not to deviate from the RD93 experiment either in equipment or design.

${ }^{1}$ Brookhaven National Laboratory; Upton, New York.

${ }^{2}$ Georgia State University; Atlanta, Georgia.

${ }^{3}$ Louisianna State University; Baton Rouge, Louisianna.

${ }^{4}$ Oak Ridge National Laboratory; Oak Ridge, Tennessee.

${ }^{5}$ University of Tennessee; Knoxville, Tennessee.

${ }^{6}$ Vanderbilt University; Nashville, Tennessee.

\section{II.C.5. Status of Data Analysis of the $\mu \mathrm{ID}$ R\&D Project}

E. C. Cornell ${ }^{6}$, E. J. Howell ${ }^{2}$, Xiaochun $\mathrm{He}^{5}$, W. Kehoe ${ }^{1}$, H. J. Kim ${ }^{4}$, P. Kirk ${ }^{3}$, J. G. $\mathrm{Kreke}^{5}$, X.T. Liu ${ }^{2}$, F. E. Obenshain ${ }^{4}$, G. A. Petitt ${ }^{2}$, S. J. Sorensen ${ }^{5}$, G. R. Young ${ }^{4}$, Ying-Chao Wang ${ }^{3}$, Zhi-Fu Wang ${ }^{3}$, Ziyang Zhang ${ }^{2}$

Analysis of the data taken for the $\mu$ ID experiment began shortly after the experiment ended, with the initial goal of determining its quantity and quality. Due to the slow timing characteristics of the Limited Streamer Mode Tube and 70 meter signal cable length to the $A D C$, the relatively weak (10 pC) signal pulse required an $A D C$ integration time of approximately $200 \mathrm{~ns}$. The signal was thus not completely distinguishable from the noise and the initial goal of analysis was to determine if any data was missed (quantity) and how well it could be distinguished from the noise (quality).

After the partially and completely lost runs were completely found, the decision was made to overhaul the analysis package in order to incorporate fortran structures. It was reported that this device worked well in other analysis packages and once learned, their advantages are striking. For instance, because 


\section{Center Strip(High Statistics)}
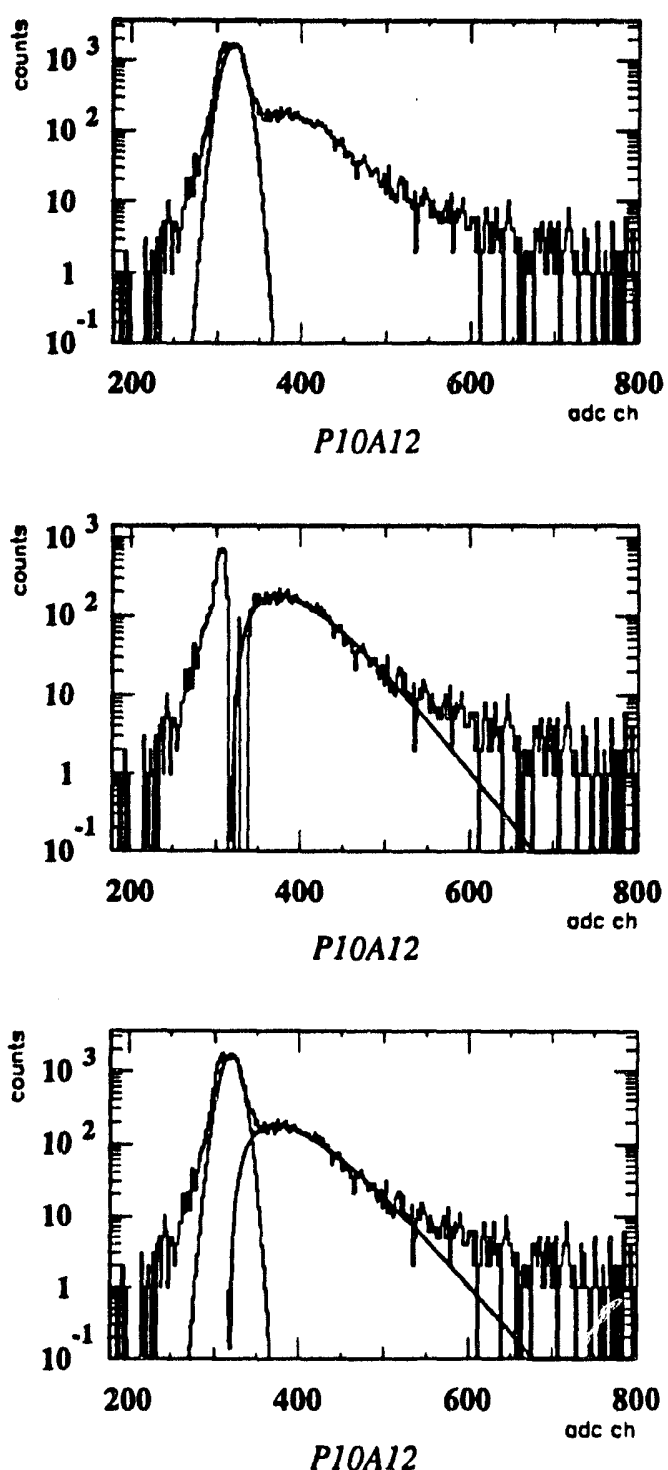

Outer Strip(Low Statistics)
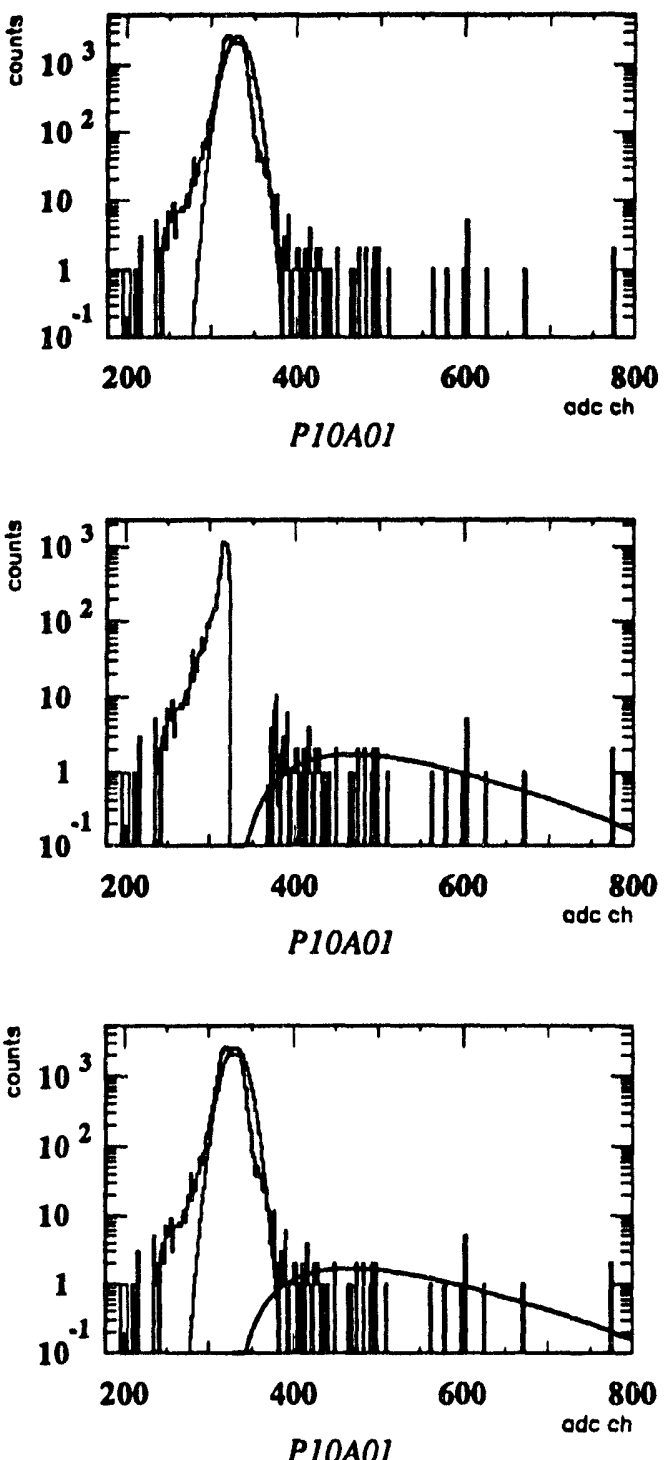

Figure II.C.5.1: Top: raw data with selected Gaussian fit to pedestal. Middle: Poisson fit to raw data after gaussian fit is subtracted. Bottom: raw data with both gaussian and poisson fits superimposed. 


\section{Comparison of 2 Methods of Pedestal Subtraction Using Hit Profiles in VERTICAL Strips for a \\ 3 GeV/c Muon Run -- Run 47}

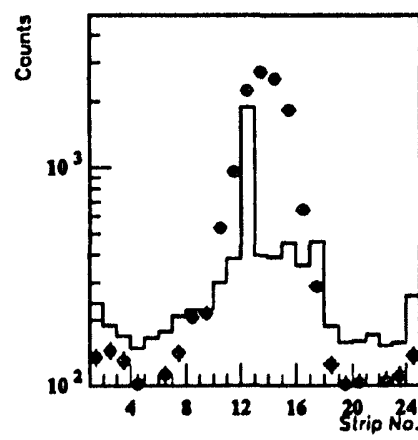

Plane 9 Vertical Strips Hit
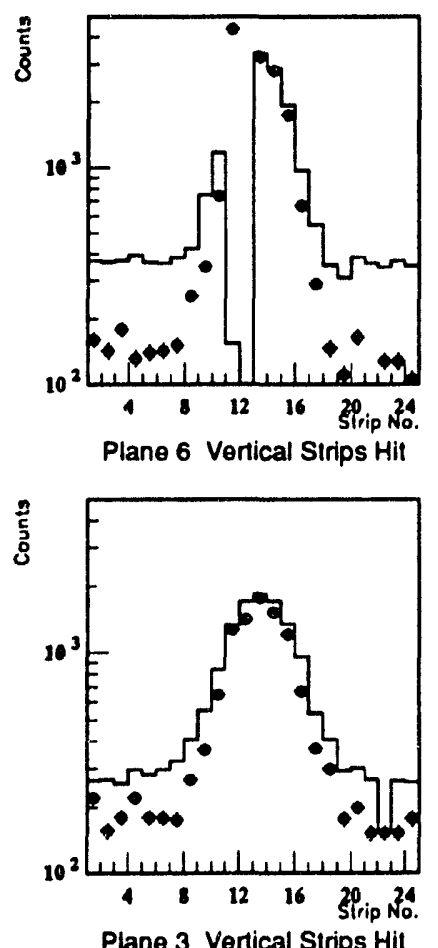

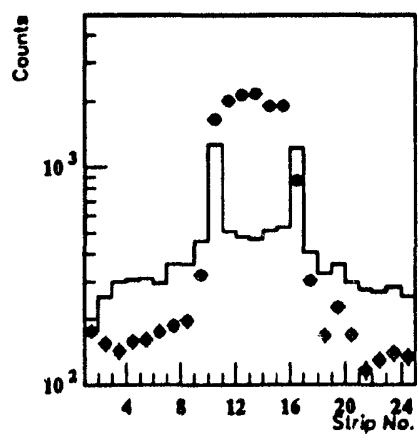

Plane 8 Vertical Strips Hit
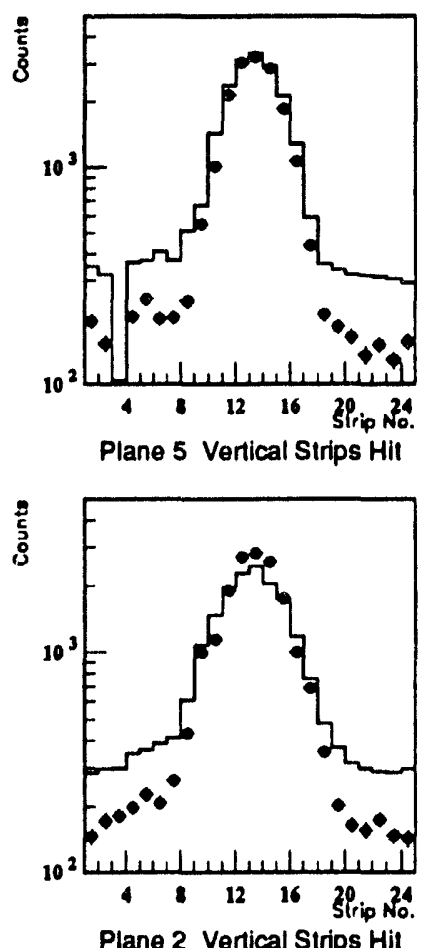
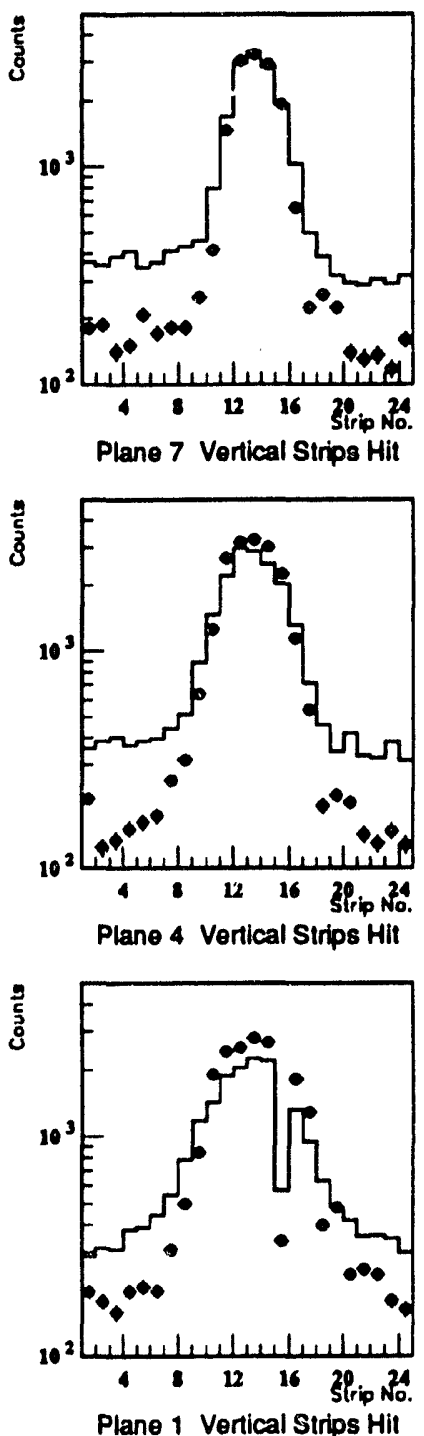

Figure II.C.5.2: Hit profiles for each vertical strip in 9 of the 10 LST planes. Method 1, shown by the bar histogram, used gaussian fits to the pedestal. Hits then had to be above the mean plus a constant (here 2.0) times the pedestal width. Method 2, shown by closed circles, used the threshold as determined by the best poisson fit to raw data above a pedestal. 
a particular channel had been addressed by a variable designated by the electronics crate number(i.e. c2), slot number(i.e. n13), and channel number(i.e. 5), altogether adc_c2_n13(5), the variable name contained no physical detector information. In using fortran structures that same parameter is stored in the variable $\mathrm{m}(2) . \mathrm{e}(5)$.adc, yielding the detector information $M \# 2$, east side, detector \#5. Changing the variable from adc_c2_n13(5) to $\mathrm{m}(2) . \mathrm{e}(5)$.adc obviously adds quite a bit of transparency to analysis.

With a working, modified analysis package, noise measurement began as simple gaussian fits to in- and out- of-beam pedestals. Comparison of these inand out-of-beam pedestals showed that the in-beam pedestal was wider and the mean shifted, clearly calling for a better method of noise measurement. For channels with good statistics, the in-beam pedestal shifted up compared to the pulser; for channels with low statistics, the in-beam pedestal shifted down. The conclusion was that we needed to accurately determine the inbeam pedestal and, moreover, to pin-point the channel where the data and pedestal merge (hereafter called the threshold). The latest scenario in pedestal measurement uses the in- and out-of-beam pedestals to define a range wherein a pedestal mean and width are chosen, incorporated into a gaussian function and subtracted from the raw data [see fig. II.C.5.1]. The remainder above the Gaussian's mean is fitted with a poisson curve which is then associated with that mean and width. Nine sets of means and widths within the defined range are chosen and the best associated poisson fit (based on mininum $\chi^{2}$ ) then selects the best mean- idth set. The method iterates 3 times, with the selected mean-width set defining a new, tighter range. The mean-width set selected at the last iteration then defines the pedestal.

There are two advantages to this method. i) The threshold is accurately determined. The intersection of the Gaussian and poisson fits sits right at the shoulder where the pedestal starts to encroach on the real data. This point, the threshold, provides the best point of separation between pedestal noise and data. 2) Using the fitted functions themselves, a range calı be defined about the threshold for minor deviations from it. Presently, the range is defined according to the ratio

$$
\frac{\text { poisson }(x)-g a u s s i a n(x)}{\text { poisson }(x)+\text { gaussian }(x)}
$$

A simple difference divided by sum. The ratio is -1.0 whele the poisson function is small (which is at the pedestal mean), 0.0 where the functions are equal (at the threshold), and +1.0 where the gaussian function is small. Since the gaussian is defined for all channels, small is defined as less than 1. 
The disadvantage of this method is computation time. Approximately 3 days on a vax 3100 are needed for one run. Another disadvantage, obstensibly, is that pedestals are not accurately determined. The pedestal fit is determined by how well a poisson curve fits the raw data. Since the in-beam pedestals broaden a little at their base, the pedestal fit gets shifted up a few channels to align the fit with the edge of this broadening [see fig. II.C.5.1]. The result is an accurate threshold measurement but inaccurate pedestal measurement. Since the pulse-heights were much lower than what we worked with in the past (order $50-100 \mathrm{mV}$ ) leaving much of the signal buried in the pedestal, accurate determination of the threshold is paramount. Figure II.C.5.2 compares the hit profiles in nine of the ten active layers of the identifier using two methods of pedestal subtraction. One method, represented by the bar histogram, measured the pedestals by fitting the pedestal with a Gaussian, then subtracting the mean value oi the gaussian and a constant times the width. Any $A D C$ value greater that that was considered "hit". The second method, represented by the closed circles, measured the threshold, as opposed to the pedestal by the method described above using poisson fits to raw data. Clearly, the second method presents a truer representation of the hit distribution through the identifier. From preliminary analysis of the data, lost and partial runs have been identified and the quality of the data appears good enough that analysis of the $\mu \mathrm{ID}$ experiment can proceed.

${ }^{1}$ Brookhaven National Laboratory; Upton, New York.

2 Georgia State University; Atlanta, Georgia.

${ }^{3}$ Louisianna State University; Baton Rouge, Louisianna.

${ }^{4}$ Oak Ridge National Laboratory; Oak Ridge, Tennessee.

5 University of Tennessee; Knoxville, Tennessee.

${ }^{6}$ Vanderbilt University; Nashville, Tennessee.

\section{II.C.6. LabVIEW Based Small Data Acquisition System}

Xiaochun $\mathrm{He}^{1}$, Joe Kreke ${ }^{1}$, Soren P. Sorensen ${ }^{1}$, Hee Kim² ${ }^{2}$, Felix E. Obenshain ${ }^{2}$, and Glenn R. Young ${ }^{2}$

Introduction: For a successful experiment, searching and developing new detector technologies have crucial impact on choosing a particular experimental setup both for the detector cost and its detection efficiency considerations. A frequent electronic signal processing is then required. Since the spring of 1993 a small cata acquisition (DAQ) system has been setup up which we have used a software tool, LabVIEW, from the National Instruments[13] and a Macintosh is UBUS plug-in card (NB-DMA-8G). This easily portable and 


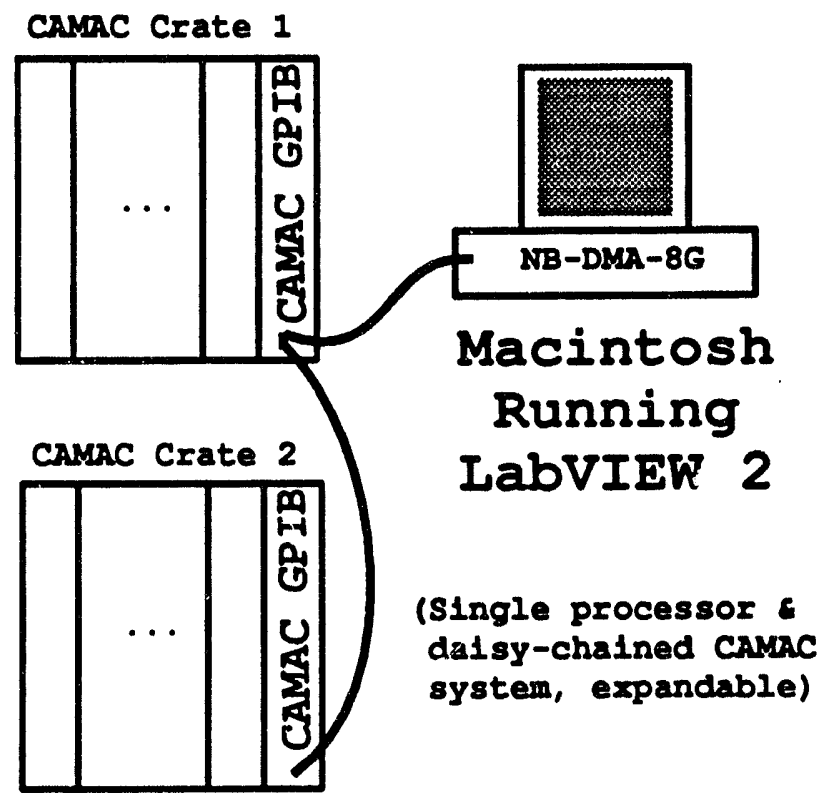

Figure II.C.6.1: Hardware layout for the small DAQsystem.

expandable system worked well for any environment which has a relatively low-rate data taking and a small number of channels. Also, this DAQ system provides a convenient way for checking signal processing modules.

LabVIEW Setup: This DAQ software is a single processor system running LabVIEW 2 on Macintosh. The data taking rate is mainly limited by the characteristic speed of LabVIEW 2. A simple connection layout is shown in Figure II.C.6.1.

The necessary and recommended hardware and software tools are:

Hardware

Macintosh (one NUBUS slot needed)

NB-DMA-8G NUBUS card

CAMAC crate (with CAMAC GPIB controller)
Software

System 7.0 or higher

LabVIEW 2.2.1 (or later version)

Think C compiler (recommended)

${ }^{1}$ University of Tennessee, Knoxville, Tennessee.

2 Oak Ridge National Laboratory, Oak Ridge, Tennessee. 


\section{VMF Crate}

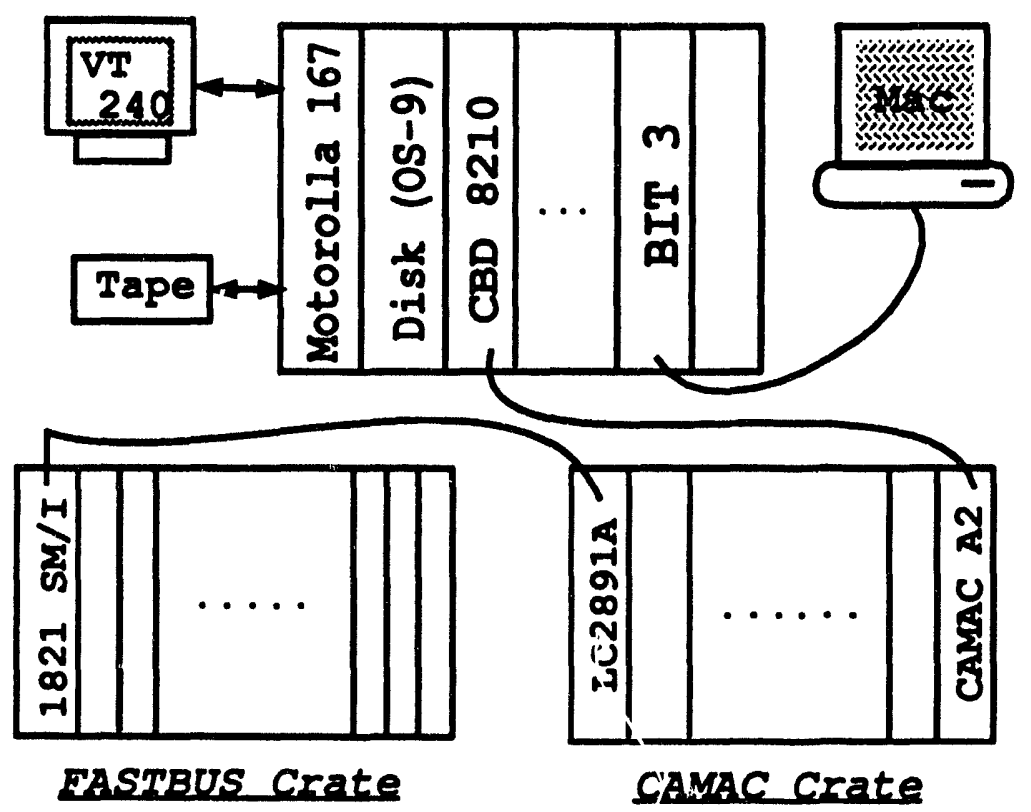

Figure II.C.7.1: The hardware layout for the RD93 DAQ system

\section{II.C.7. RD93 Data Acquisition System}

Authors list is the same as in II.C.6.

A Motorolla 167 (with MC68040 processor) running os-9 was adapted as the main data acquisition (DAQ) engine for a PHENIX R\&D experiment (RD93) in last summer at Brookhaven National Lab (BNL). The overall DAQ structure was originally made by Sean McCorkle from $\mathrm{BNL}$, and modified by our group to include FASTBUS readout interface. The hardware connection is shown in Figure II.C.7.1. The main engine (Motorolla 167 running OS-9) waits for VME interrupts (coming from front-end electronics to CBD 8210 , NIM level), reads outs the digitized electronic signals and assembles event records, and then write the event record to tape. If the space permits, the event records are also copied to a Bit3 dual-port memory for monitoring by Mac. A VT 420 terminal connecting to the Motorolla 167 via RS-232 lines acts as the main control console during run and provides the access to the os-9 system. Under normal data-taking condition, the Mac gets a fraction of event records and histograms the selected channels by LabVIEW 2. The access to the CAMAcsystem is 
through CBD8210 (a CES product) connected to a CAMAC A2 controller. The further access to the FASTBUS is via an CAMAC-FASTBUS interface (LC 2891A) which sits on CAMAC crate.

This DAQ software is a multi-process system (see Figure II.C.7.2). The main component is the Run control - it is running in place of the shell environment on console terminal. Besides reading in simple commands and updating the screen display, it also starts the "main acquisition engine" which consists of the following processes:

- Fetcher - reads out the front-end electronics when there is trigger, and builds an event record in the circular buffer called cbuff.

- Recorder - reads out event records from the cbuff. If the user is recording the data, then each buffer is written to tape. It also transmit a fraction of data to a smaller circular buffer in Bit3 dual-port memory for monitoring by Mac.

- Intercom - a shared memory block for passing informatioz. back and forth between the run-control and the engine processes.

This DAQ system has a high data taking rate. There were about 20 physics events recorded per second on average for about 800 readout channels during last summer's test beam run. The only part which lags its integrated performance is the online monitoring via LabVIEW running on Mac. The LabVIEW[13] is just too slow for processing and displaying the data. A new online monitoring scheme is now under developing in the moment of preparing this report.

\section{II.C.8. Overview of WA80 Progress}

WA80 Collaboration: R. Albrecht ${ }^{1}$, T. C. Awes ${ }^{6}$, P. Beckmann ${ }^{2}$, F. Berger ${ }^{2}$, R.Bock ${ }^{1}$, G. Cleasson ${ }^{3}$, G. Clewing ${ }^{2}$, L. Dragon ${ }^{2}$, A. Eklund ${ }^{3}$, R. L. Ferguson ${ }^{6}$, A. $\mathrm{Franz}^{5}$, S. Garpman ${ }^{3}$, R. Glasow ${ }^{2}$, H. A. Gustafsson ${ }^{3}$, H. H. Gutbrod ${ }^{1}$, J. $\mathrm{Idh}^{3}$, P. Jacobs $^{4}$, K.-H. Kampert ${ }^{2}$, B. W. Kolb ${ }^{1}$, P. Kristiansson ${ }^{3}$, I. Y. Lee ${ }^{6}$, H. Lohner ${ }^{2}$, I. Lund $^{1}$, F. E. Obenshain ${ }^{6}$, A. Oskarsson ${ }^{3}$, I. Otterlund ${ }^{3}$, T. Peitzmann ${ }^{2}$, S. Persson ${ }^{3}$, F. Plasil ${ }^{6}$, A. M. Poskanzer ${ }^{4}$, M. Purschke ${ }^{2}$, H.-G. Ritter ${ }^{4}$, S. Saini ${ }^{6}$, R. Santo ${ }^{2}$, H. R. Schmidt ${ }^{1}$, T. Siemiarczuk ${ }^{1}$, S. P. Sorensen ${ }^{5}$, E. Stenlund ${ }^{3}$, M. L. Tincknell ${ }^{6}$, G. R. Young ${ }^{6}$, and $\mathrm{X} . \mathrm{He}^{5}$

The WA 80 experiment was the first high energy heavy-ion experiment UT participated in and it continues to be ihe workhorse for providing "physics" 


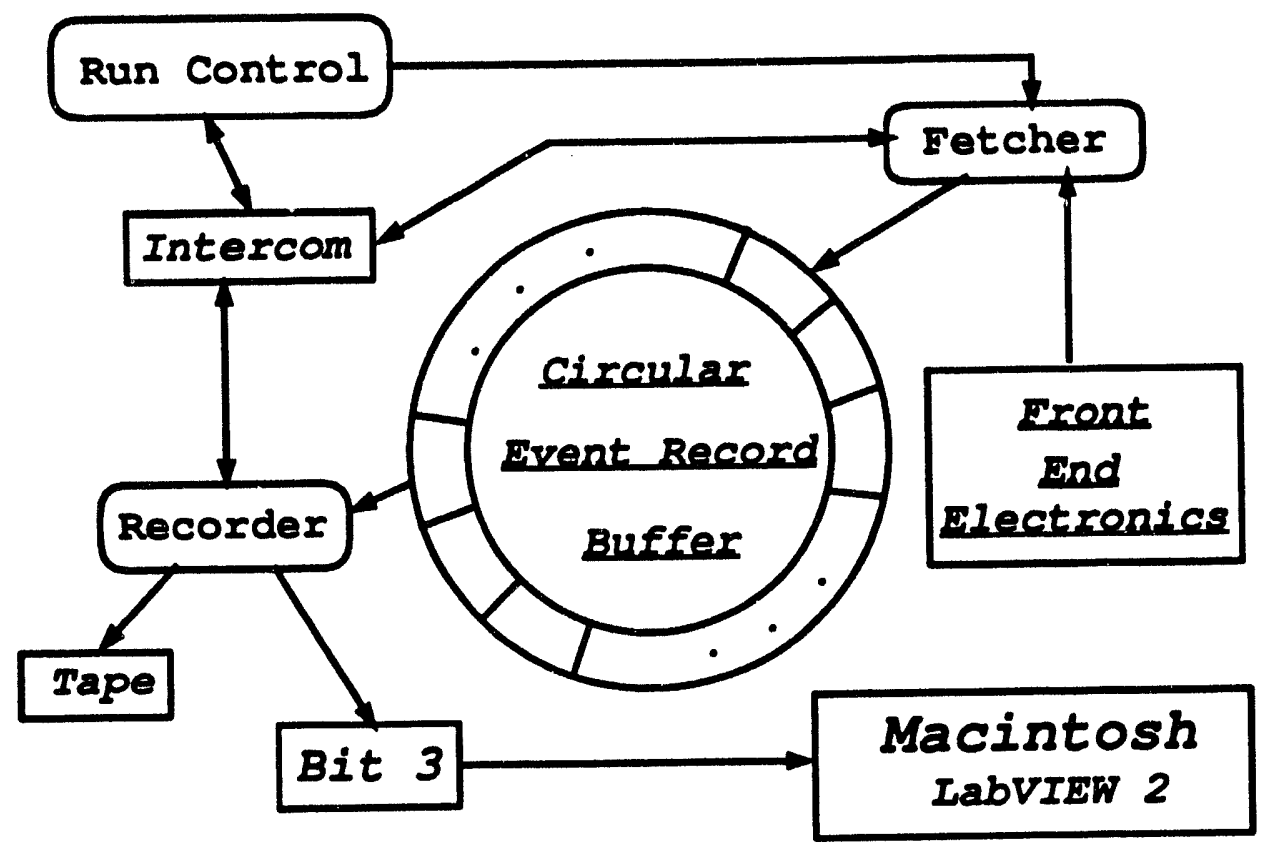

Figure II.C.7.2: The software logic for the RD93 DAQ system

output even if the data collection finished five years ago. In the following is described the highlights of this years analysis.

The "event-mixing" technique, enabling the precise determination of background events, was applied to the high-statistics photon data from ${ }^{32} S$ reactions with ${ }^{32} S$ and ${ }^{197} \mathrm{Au}$ at $200 \mathrm{GeV} /$ nucleon. It was possible to obtain meaningful reconstructed hadron results down to signal/background ratios of 0.002 . The production of $\eta$ mesons was determined in the $\mathrm{p}_{T}$ range from 0.5 to $3 \mathrm{GeV} / \mathrm{c}$. These results were found to be consistent with phenomenological $\mathrm{mT}$-scaling previously observed in hadron-hadron and hadron-nucleus reactions. $\eta / \pi^{\circ}$ ratios vs. $\mathrm{p}_{T}$, obtained from a large number of different reactions at $E / A=200 \mathrm{GeV}$, were found to fall on a universal curve.

The $\pi^{\circ}$ and $\eta$ reconstruction capabilities, toget her with higher-statistics data, improved shower-reconstruction algorithms, and other data analysis improvements, lead to the overall reduction of the errors associated with the extraction single-photon results from $15 \%$ to about $6-7 \%$ in the $\mathrm{p}_{T}$ range of 0.3 to $1.5 \mathrm{GeV} / \mathrm{c}$. A slight, but statistically significant, excess of photons over the number of photons accounted for by hadronic decays was observed for 
central (and minimum bias) events in the $\mathrm{p}_{T}$ range 0.5 to $2.0 \mathrm{GeV} / \mathrm{c}$ for $\mathrm{S}+$ $\mathrm{Au}$ at $200 \mathrm{GeV} /$ nucleon. No such photon excess was observed in peripheral events.

Local slopes of $\pi^{\circ}$ spectra were extracted as a function of $\mathrm{p}_{T}$ and of collision centrality for $\mathrm{S}+\mathrm{Au}$ reactions at $200 \mathrm{GeV} /$ nucleon. For central events a monotonic increase of the slope parameters was observed ranging $\mathrm{f}$. om about $200 \mathrm{MeV} / \mathrm{c}$ at $\mathrm{p}_{T}=0.5 \mathrm{GeV}$ to $250 \mathrm{MeV} / \mathrm{c}$ at $\mathrm{p}_{T}=3.0 \mathrm{GeV}$. A similar monotonic increase was found for the slope parameters of peripheral events, but their absolute values, in this case, were found to be consistently about $20 \mathrm{MeV} / \mathrm{c}$ below those obtained from central collisions. The peripheral reaction results were found to be similar to those obtained from pp reactions.

The target fragmentation region $(-1.7<\eta<1.3)$ has been studied in $\mathrm{p}+\mathrm{A}$ and ${ }^{16} \mathrm{O}+\mathrm{A}$ reactions at 60 and $200 \mathrm{GeV} /$ nucleon with the Plastic Ball. Azimuthal correlations, as well as two-particle correlations fo protons and pions, were obtained. Source radii extracted from pp-correlations were found to follow a $A^{1 / 3}$ dependence. This indicates that the entire target nucleus is involved in the reaction, even in the case of $p$-induced reactions. Thus, a simple target-spectator picture does not seem to apply at these energies. A paper is in the final stages of preparation.

A prototype $8 \times 8$ module high-resolution electromagnetic BGO calorimeter with longitudinal segmentation was exposed to pions and electrons with momenta ranging from 0.5 to $6 \mathrm{GeV} / \mathrm{c}$ at the CERN PS. The crystals were read out via large-area silicon PIN photodiodes coupled to ORNL-developed lownoise preamplifiers and pulse-processor modules with automatic gain-range selection. The energy and position resolution as well as the performance for particle identification and two-shower separation were studied. The energy resolution was found to be better than $1 \%$ at energies above $4 \mathrm{GeV}$. The measured position resolution can be parameterized by $\sigma_{x}=3.3 / \sqrt{E} \mathrm{~mm}$. A paper has been submitted for publication.

${ }^{1}$ Gesellschaft für Schwerionenforschung (GSI), Darmstadt, Germany.

2 University of Münster, Germany.

3 University of Lund, Sweden.

${ }^{4}$ Lawrence Berkeley Laboratory, Berkeley, California.

${ }^{5}$ University of Tennessee, Knoxville, Tennessee.

${ }^{6}$ Oak Ridge National Laboratory, Oak Ridge, Tennessee. 


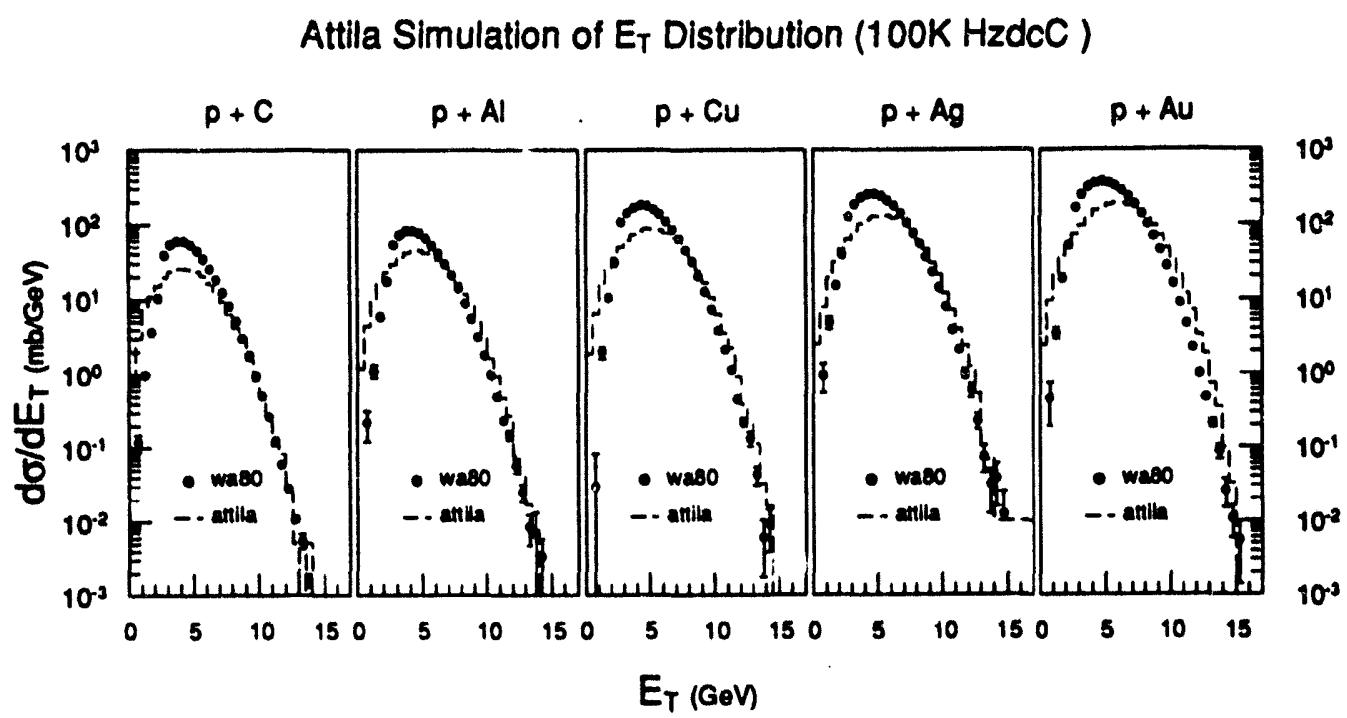

Figure II.C.9.1: Transverse energy spectra for $200 \mathrm{GeV}$ proton induced reactions on ${ }^{12} \mathrm{C},{ }^{27} \mathrm{Al},{ }^{\text {nat }} \mathrm{Cu},{ }^{n a t} \mathrm{Ag}$, and ${ }^{197} \mathrm{Au}$. The full circle points are from our measurement, the histograms from Attila simulation.

\section{II.C.9. Status Report of WA80 Proton Data Analysis WA80 Collaboration (see Sec. II.C.8.}

Since last year's progress report of the WA80 proton data analysis [14], extensive Monte Carlo (MC) simulations have been carried out to compare theoretical results with our data. Those MC codes like Attila, Fritiof, Marco, Mcfm and Venus all have in common that, in first order, incoherent superposition of nucleon-nucleon interactions is used to simulate nucleus-nucleus cases. It will be very important to see how well these codes predict the proton-nucleus collisions.

We have compared some of our data with predictions from Attila, Fritiof 1.7, Marco, ihcfm and Venus 3.11 (with and without rescattering) code. We have found that it is very important to simulate the experimental trigger conditions correctly in order to have a closer fit to the data. Since we had a forward energy threshold cut (measured by ZDC) integrated into the online trigger those very peripheral events were cut away. Overall, by carefully simulating the trigger condition, the Attila code gives a better fit to our data comparing with other codes mentioned here especially for the $d \sigma / d E_{T}$ distribution (see Figure II.C.9.1).

We have also compared our $d E_{T} / d \eta$ data with the predictions of these 


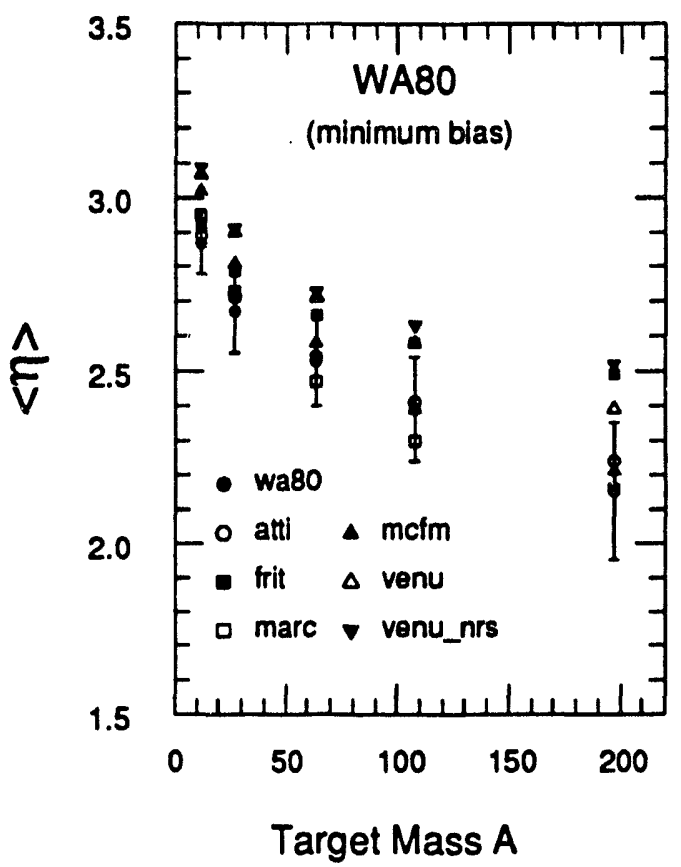

Figure II.C.9.2: Comparison of $\langle\eta\rangle$ vs. target mass between WA80 data and Monte Carlo simulations.

codes. Figure II.C.9.2 shows a compilation of simulated results together with our data. The vertical axis is the $\eta$ centroid extracted from Gaussian fit to the pseudorapidity distributions, and the horizontal axis represents the target mass. It is clearly seen that all of the codes show a correct trend of the $\eta$ centroid dependence on the target mass. However, Attila, Marco and Mcfm codes give a better fit to our data compared with Fritiof and Venus fits. Our data also shows that there is little difference between Venus with rescattering and without rescattering. It starts showing up some differences for the $\mathrm{Au}$ target case.

At this stage, the overall proton data analysis is completed. All the important results have been put into a paper (draft 2.0) for publication in near future, which is under reviewed by our group members for the moment. The main conclusions are summarized below:

The transverse energy $\left(E_{T}\right)$ distribution of $200 \mathrm{GeV} / \mathrm{c}$ proton induced reactions increases with increasing target mass for a given measured $E_{T}$. At high $E_{T}$ end, the $E_{T}$ production shows a saturation trend starting from $\mathrm{Ag}$ target case. The maximum $E_{T}$ measured is about $16 \mathrm{GeV}$. 
The $E_{T}$ pseudorapidity distribution shows the energy flow in pseudorapidity space. A Gaussian fit to our data clearly displays a strong target mass dependence, especially in the target rapidity region. The average pseudorapidity $\langle\eta\rangle$ shifts backward as increasing the target mass. In the projectile rapidity region, our data shows some degree of energy slow depletion for a relatively heavier target mass. This depletion is considered as a reflection of the conservation of energy. No $\langle\eta\rangle$ backward shifting effect observed for different $E_{T}$ bin slices. It stays constant.

A convoluted gamma distribution model fits our data well. It suggests that the inclusion of the energy degradation of the incident proton passing through a target nucleus is needed. The fraction of energy loss of the incident proton for each successive collisions is about $60 \%$, and the average transverse energy produced in the first binary collision is around $3.5 \mathrm{GeV}$ for the pseudorapidity coverage of $2.4<\eta<5.5$.

The Attila MC code gave a relatively better fit to our data comparing with other MC codes (Fritiof, Venus, Mcfm, Marco) which indicates that an accurate nuclear geometry should be used while making any quantitative theoretical calculations. Furthermore, our comparison shows that it is equally important that a right trigger condition should be simulated in any $\mathrm{MC}$ code.

\section{II.C.10. Azimuthal asymmetry of pion emission in proton-nucleus reactions at $200 \mathrm{GeV}$}

WA80 Collaboration (see Sec. II.C.8.)

The study of the target fragmentation region in high energy protonnucleus and nucleus-nucleus interactions has provided conclusive evidence for a strong participation and global excitation of the target nucleus. Experimentally, this finding is based both on the linear rise of the baryon yield with target mass [15] as well as on the linear rise of the pp-correlation radius parameter with target size [16]. Additional information on the mechanism of target excitation can be gained from global azimuthal correlations of protons and pions. In Ref. [17] the azimuthal correlation function was defined by the relative opening angle between the transverse momentum vector sum of particles emitted forward and backward with respect to the rest frame of the target nucleus $\left(y_{t} \approx 0.2\right)$. For the first time it could be shown that pions in the target fragmentation region of $4.9,60$, and $200 \mathrm{GeV} \mathrm{p}+\mathrm{Au}$ collisions are preferably emitted side-by-side. These results were interpreted as an indication for strong pion interactions and $\Delta$-formation in the target nucleus [18].

To verify this interpretation, the analysis has been extended both to im- 

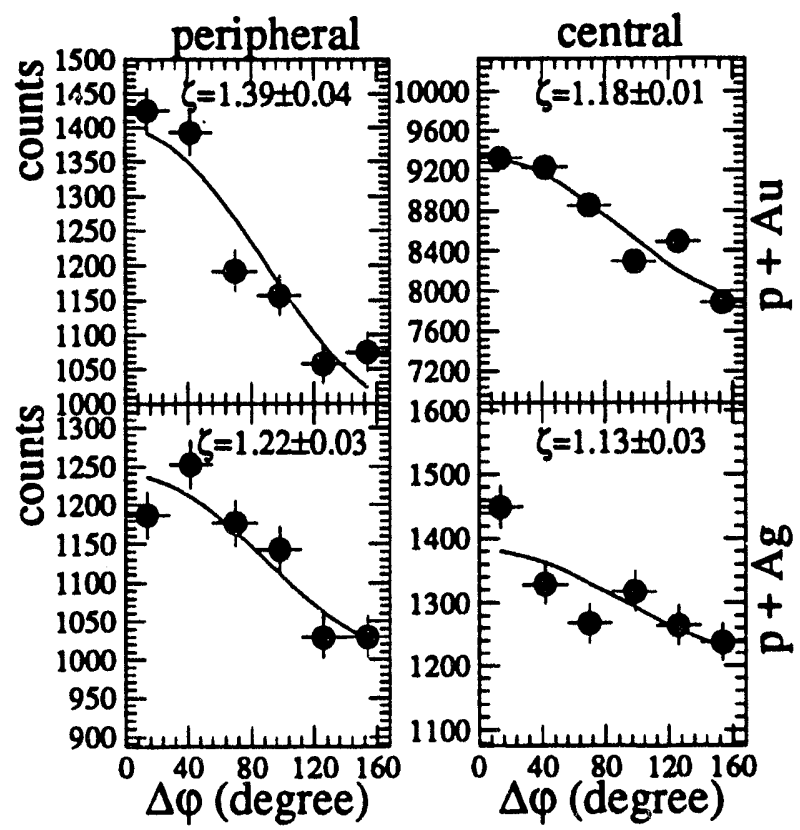

Figure II.C.10.1: Azimuthal correlation functions of positive pions measured with the Plastic-Ball in the pseudorapidity range $-1.7 \leq \eta \leq 1.3$. The upper and lower graphs show results obtained for $200 \mathrm{GeV} \mathrm{p}+\mathrm{Au}$ and $\mathrm{p}+\mathrm{Ag}$ interactions, respectively, and the left and right panel selections of semi-peripheral and semi-central events, respectively.

pact parameter selected data as well as to lighter target nuclei. Figure II.C.10.1 shows the azimuthal correlation functions of positive pions for peripheral and central $200 \mathrm{GeV} \mathrm{p}+\mathrm{Au}$ and $\mathrm{p}+\mathrm{Ag}$ reactions. All distributions show the expected peak at $\Delta \varphi=0$ and thereby demonstrate the side-by-side emission of pions. To quantify the strength of the correlation, the maximum-to-minimum ratio $\zeta$ has been extracted by fitting a $\cos (\Delta \varphi)$ distribution to the data and its results are also given in the figure. Comparing the numbers, we observe an increasing correlation stirength both with increasing impact parameter and increasing target mass. Such a behavior is in fact expected in a geometrical picture as is behind the absorption interpretation discussed above.

The azimuthal correlation at $\Delta \varphi=0$ can be interpreted as direction of the reaction plane and allows to study observables in-plane and out-of-plane. As an example, figure II.C.10.2 shows the ratio of the pion kinetic energy spectra in plane divided by out-of-plane. This ratio increases significantly with increasing pion energy, i.e. the in-plane energy distribution is harder than the out-of-plane distribution. An apparently inverse behavior has been observed 


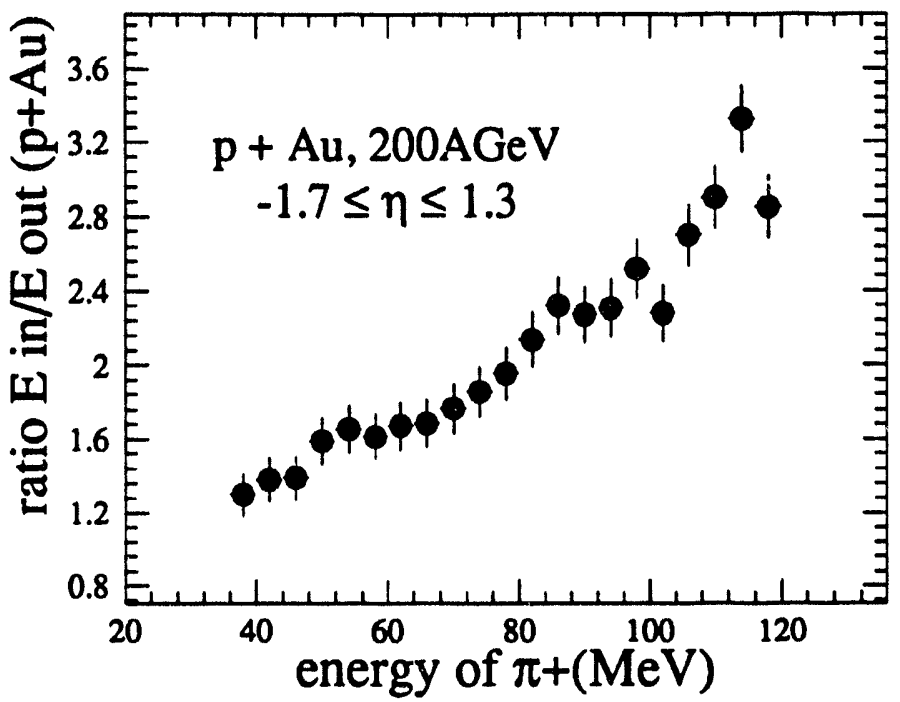

Figure II.C.10.2: Ratio of the pion kinetic energy spectra in-plane to out-ofplane in min. bias $\mathrm{p}+\mathrm{Au}$ interactions at $200 \mathrm{GeV}$.

at SIS [19] but for higher pion energies. However, taking into account the different geometry of $\mathrm{pA}$ and AA collisions, the two results are consistent: in both cases one can think of nuclear matter absorbing the pions and subsequent remission at lower average kinetic energies.

\section{II.C.11. Particle Spectra from Ultrarelativistic Collisions at Back- ward Angles}

WA80 Collaboration (see Sec. II.C.8.)

Cumulative particle production refers to the mechanism of particle emission into the backward laboratory direction $\left(\Theta_{l a b}>90^{\circ}\right)$ in hadron-nucleus and relativistic heavy-ion collisions [20]. Two different processes can be thought of which lead to the emission of cumulative particles: rescattering amongst target spectator matter as a non-local effect on the one hand, and collisions with possible high-mass objects in the hot core of the reaction system as a local effect on the other hand. In order to be able to distinguish between those, different projectile-target combinations from the experiment, were analyzed.

Protons emitted into the angular range $30^{\circ}<\Theta_{l a b}<160^{\circ}$ were measured in the Plastic Ball detector [21] in the setup of the WA80 experiment at CERN 


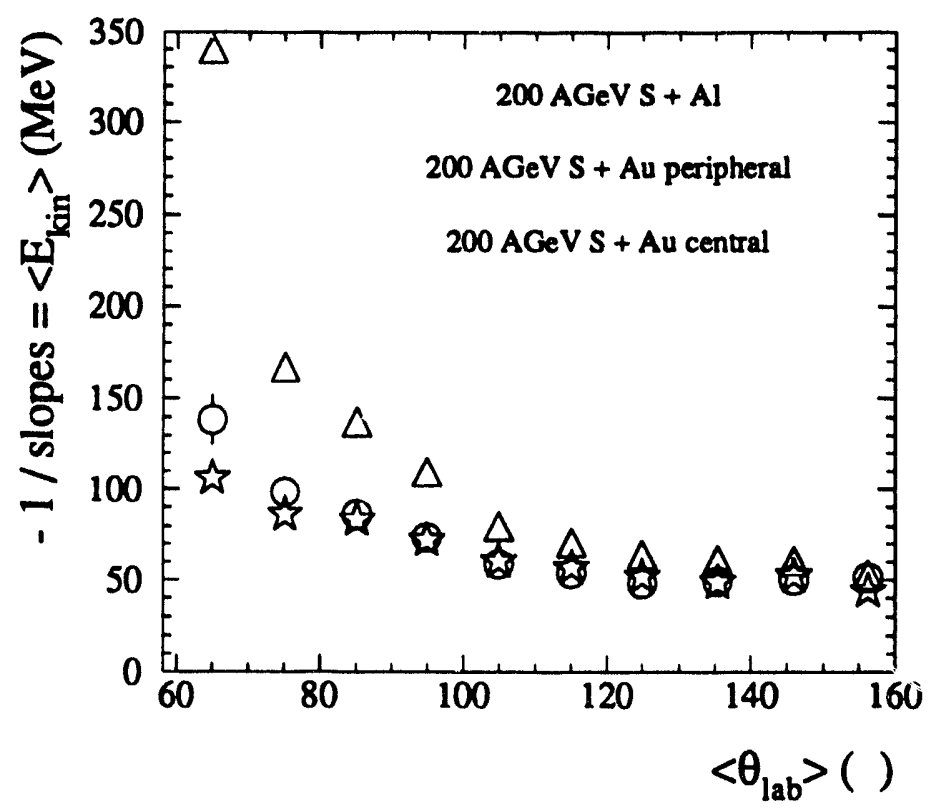

Figure II.C.11.1: Target mass dependence of the slope parameters extracted from proton cross sections as a function of $\langle\Theta\rangle$ from $200 \mathrm{AGeV} \mathrm{S}$ induced reactions. The circles correspond to the $\mathrm{Al}$ target measurements, the triangles to central $\mathrm{S}+\mathrm{Au}$ collisions, and the stars to peripheral $\mathrm{S}+\mathrm{Au}$ reactions.

in 1986-1987. Out of the whole data sample of $200 \mathrm{AGeV}{ }^{16} \mathrm{O}$ and ${ }^{32} \mathrm{~S}$ induced reactions, very central and peripheral events were selected by means of the fraction of beam energy in the WA80 ZDC (Zero-Degree-Calorimeter) [22]. For symmetric systems, the central selection maximizes the overlap of the projectile with the target and minimizes possible rescattering in the target spectator region as source of cumulative particles.

The target mass dependence was analyzed by comparing the proton spectra of the light, almost symmetric system $200 \mathrm{AGeV}^{32} \mathrm{~S}+{ }^{27} \mathrm{Al}$ with the asymmetric system ${ }^{32} \mathrm{~S}+{ }^{197} \mathrm{Au}$. From the xponential - shaped proton spectra, the negative inverse slopes were extracte and the results are summarized in Fig. II.C.11.1. The slope parameters, wh: h represent a mean kinetic energy of the emitted protons, of $\mathrm{S}+\mathrm{Al}$ (circles) are shown together with those of central (triangles) and peripheral (stars) $\mathrm{S}+\mathrm{Au}$ collisions. For the backward region, the proton mean kinetic energies of peripheral $\mathrm{S}+\mathrm{Au}$ match those of $\mathrm{S}+\mathrm{Al}$, which may indicate that the same mechanisms are responsible for cumulative protons in these reactions.

The energy dependence of cumulative proton production was investigated 


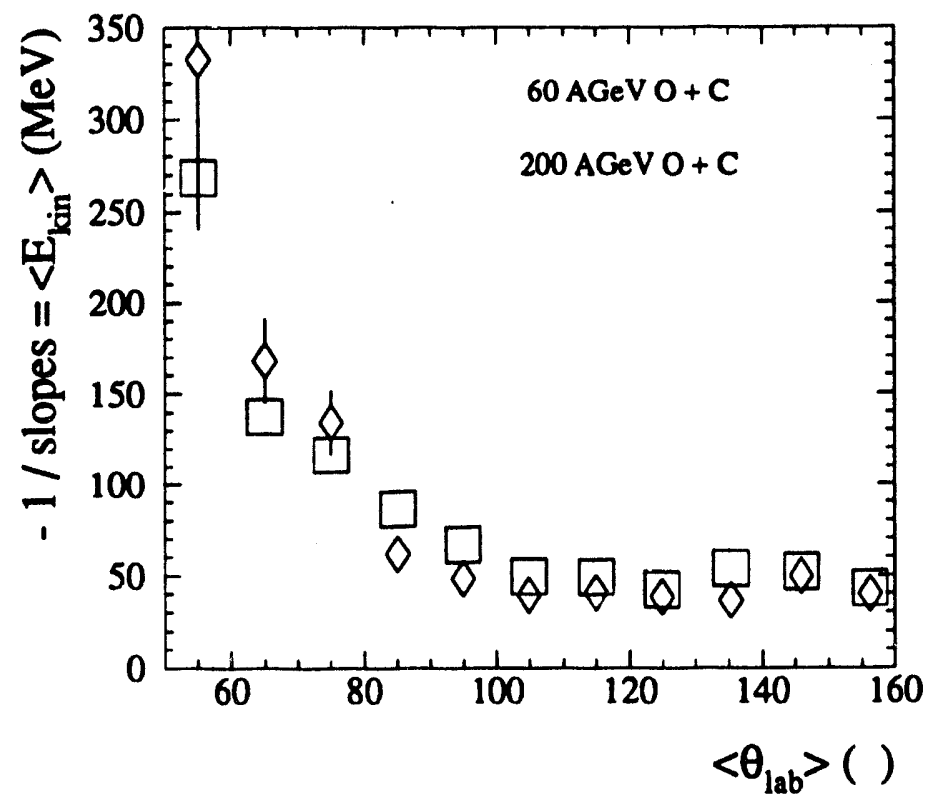

Figure II.C.11.2: Projectile energy dependence of the slope parameters as a function of $\langle\Theta\rangle$. The squares denote $200 \mathrm{AGeV} \mathrm{O}$ induced reactions, the diamonds $60 \mathrm{AGeV} O$ induced reactions. The target was in both cases ${ }^{12} \mathrm{C}$.

by comparing central collisions of the system $\mathrm{O}+\mathrm{C}$ for two projectile energies: $200 \mathrm{AGeV}$ and $60 \mathrm{AGeV}$. The centrality criteria in these cases were the following: for $200 \mathrm{AGeV} \mathrm{O}+\mathrm{C}$, only events with not more than $45 \%$ of the whole beam energy in the ZDC were called central. For $60 \mathrm{AGeV}$ oxygen induced reactions, the condition for centrality was not more than $20 \%$ of the whole energy available in the ZDC. Both correspond to an impact parameter below $1 \mathrm{fm}$, according to calculations with the event generator FRITIOF. In Fig. II.C.11.2, the results for the slope parameters are shown: in the backward hemisphere, both systems exhibit similar behavior, which indicates that proton emission into the backward hemisphere in ultrarelativistic heavy-ion reactions is mostly independent of the projectile's energy.

These findings suggest that cumulative proton production at backward angles in ultrarelativistic heavy-ion collisions is due to local phenomena. 


\section{II.C.12. Overview of WA93 Progress}

WA93 Collaboration: P. Donni ${ }^{1}$, R. Glasow ${ }^{7}$, E. Durieux ${ }^{1}$, G. Holker ${ }^{7}$, M. Izycki $^{1}$, K.-H. Kampert ${ }^{7}$, M. Martin ${ }^{1}$, T. Peitzmann ${ }^{7}$, L. Rosselet ${ }^{1}$, M. Purschke , N. Solomey $^{1}$, R. Santo ${ }^{7}$, R. Bock ${ }^{2}$, K. Steffens ${ }^{7}$, H. H. Gutbrod ${ }^{2}$ P. Steinhauser ${ }^{7}$, B. W. Kolb $^{2}$, D. Stuken ${ }^{7}$, H. R. Schmidt ${ }^{2}$, T. C. Awes ${ }^{1}$ 0, S. Kachroo ${ }^{3}$, F. E. Obenshain ${ }^{1}$, N. K. Rao ${ }^{3}$, F. Plasil ${ }^{10}$, S.S. Sambya ${ }^{13}$, S. Saini ${ }^{10}$, V. Antonenko ${ }^{4}$, G. R. Young ${ }^{10}$, S. Fokin ${ }^{4}$, M. M. Agarwal ${ }^{8}$, M. Ippolitov ${ }^{4}$, V. S. Bhatia ${ }^{8}$, K. Karadjev4, I. S. Mittra $^{8}$, A. Lebedev ${ }^{4}$, B. S. Garcha ${ }^{8}$, V. Manko ${ }^{4}$, K. B. Bhalla ${ }^{9}$, S. Nikolaev ${ }^{4}$, S. K. Gupta ${ }^{9}$, A. Vinogradov ${ }^{4}$, S. Lokanathan ${ }^{9}$, H. Lohner ${ }^{5}$, S. Mukherjee ${ }^{9}$, I. Lund $^{5}$, S. Ramiwala ${ }^{9}$, G. Claesson ${ }^{6}$, X. C. He, A. Eklund ${ }^{6}$, S. P. Sorensen, S. Garpman $^{6}$, N. Van Eijndhoven ${ }^{12}$, H. A. Gustafsson ${ }^{6}$, R. Kamermans ${ }^{12}$, J. Idh ${ }^{6}$, S. Chattopadhyay ${ }^{13}$, A. Oskarsson ${ }^{6}$, A. Das ${ }^{13}$, I. Otterlund ${ }^{6}$, M.R.D. Majumdar ${ }^{13}$, K. Soderstrom ${ }^{6}$, T. Ghosh ${ }^{13}$, E. Stenlund ${ }^{6}$, G.S.N. Murthy ${ }^{13}$, H. J. Whitlow ${ }^{6}$, M. N. Rao ${ }^{13}$, F. Berger ${ }^{7}$, B. C. Sinha ${ }^{13}$, D. Bock ${ }^{7}$, D. K. Srivastava ${ }^{13}$, G. Clewing ${ }^{7}$, M. D. Trivedi ${ }^{13}$, L. Dragon ${ }^{7}$, Y. P. Viyogi ${ }^{13}$.

The WA93 experiment is the successor to WA80. The goals are to obtain higher-statistics photon data and to serve as a prototype setup for a future experiment (WA98) that will take advantage of SPS lead beams. A large magnet (GOLIATH) and multistep avalanche counters (MSAC's) have been added for the purpose of tracking charged particles over a wide angular range. A photon multiplicity detector (PMD), consisting of a lead converter backed by scintillating tiles, was also implemented. MIRAC and the ZDC were retained from WA80 and continued to be the combined responsibility of the UTK and ORNL group. Two WA93 runs have taken place, one each in 1991 and 1992. A large amount of photon data have been obtained, and analysis is in progress.

Analysis of WA93 data will continue. The large-statistics data sample is expected to lead to a further reduction of errors associated with the extraction of single-photon results. A search for the production of mesons other than $\pi^{\circ}$ and $\eta$ are planned.

1 University of Geneva, Switzerland.

${ }^{2}$ GSI, Darmstadt, Germany..

3 University Jammu, India.

${ }^{4}$ Kurchatov Inst., Moscow, USSR..

${ }^{5}$ KVI, Groningen, The Netherlands..

${ }^{6}$ University of Lund, Sweden..

7 University of Munster, Germany..

8 Punjab University, Chandigarh, India..

9 University Rajasthan, Jaipur, India..

${ }^{10}$ Oak Ridge National Laboratory..

11 University of Tennessee.. 
12 University of Utrecht, The Netherlands.

13 VECC, Calcutta, India..

\section{II.C.13. Overview of WA98 Progress}

WA98 Collaboration (see Sec. II.C.12.)

The WA98 is the successor to the WA80 and WA93 experiments and is designed to take advantage of the lead beams which are scheduled to become available in November, 1994. It is based on the experience obtained in WA93 and consists of mostly the same subsystems. The lead glass array of 4000 modules is being expanded to 10,000 modules. ORNL is responsible for the development of monolithic electronics for the 10,000 lead glass detectors. The ZDC calorimeter is being replaced by a new device built by Russian collaborators. MIRAC will be retained, and it remains the combined responsibility of UTK/ORNL. It will very probably provide the most reliable event-characterization capability of WA98. MIRAC, together with another calorimeter recuperated from an earlier experiment, will provide WA98 with the capability to measure transverse-energy production over a reasonably large range of pseudorapidity. WA98 appears to be the only experiment to have the capability to make such measurement with lead beams.

In the summer of 1993 UTK/ORNL collaboration members did undertake the recalibration of all MIRAC modules in the SPS test beam. Following this, MIRAC was disassembled into its component stacks and reassembled in its final location. It was the first WA98 subsystem to be located in the reconfigured WA98 experimental area.

During 1993 we have been involved in revisions of the MIRAC analysis software and have performed calculations for the values of transverse energy trigger weights. In 1994 we plan a major upgrade of all MIRAC software in order to be able to provide transverse energy spectra as soon as possible after the start of data collection in November.

\section{II.C.14. The Photon Spectrometer in the WA98 Experiment}

WA98 Collaboration (see Sec. II.C.12.)

One of the major aims of the WA98 experiment [23] with $\mathrm{Pb}+\mathrm{Pb}$ collisions at $160 \mathrm{~A} \mathrm{GeV}$ is the measurement of direct photons as a signal for the phase transition to the quark-gluon plasma and as a temperature probe for the hot hadronic system in these collisions. For this purpose a 10000 module 


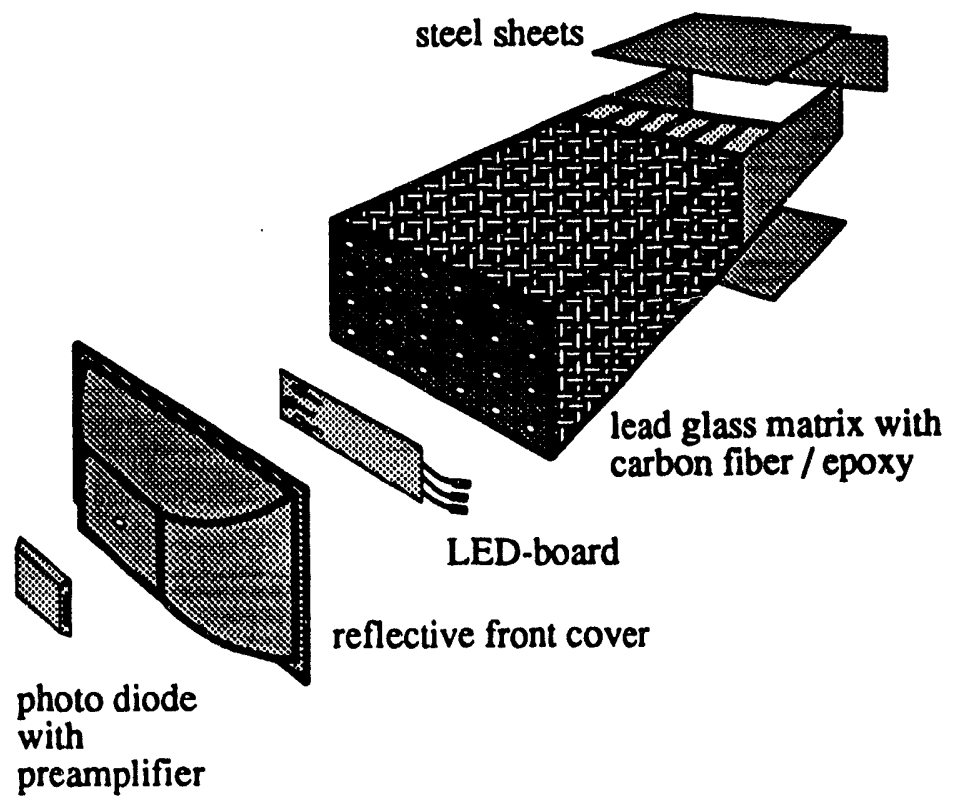

Figure II.C.14.1: Schematic front view of a Supermodule

lead glass calorimeter is currently under construction. This photon spectrometer will consist of two sections mounted below and above the beam tube at a. distance of 21.5 meters from the target.

To allow flexible arrangement of the detector, a design based on subunits (Supermodules) of $6 \times 4$ lead glass blocks was chosen. Plastic PMT housings are glued to the blocks, the glass is wrapped with aluminized mylar and shrinktube. The 24 modules are glued together with carbon fiber and epoxy. Steel sheets used for a metal housing of the PMTs and bases are incorporated in this structure. Figure II.C.14.1 shows a front view of the mechanics of the Supermodules. The stacked Supermodules will thus form a large detector with as little dead material as possible.

Each of these Supermodules will be equipped with its own gain monitoring system based on LED pulsers. One yellow LED is fed by an avalanche pulse generator, which yields PMT pulse widths of $30 \mathrm{~ns}$. These pulses are very similar to electronic pulses originating from Čerenkov light. Short term variations of the pulse amplitude have been shown to be dominantly due to photoelectron number fluctuations. Two other LEDs (one yellow, one blue) with independent pulse generators are used for control of the linearity and changes in the frequency response of the PMTs and for additional cross checks. 


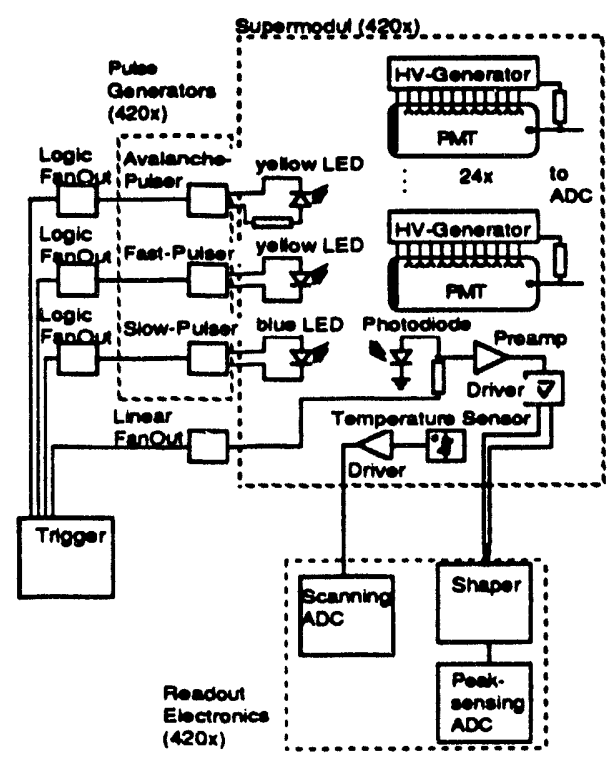

Figure II.C.14.2: Schematic layout of the monitoring system electronics

The LEDs are mounted on the plastic front face of the Supermodules emitting their light towards the inner surface of a plastic cover. This surface is covered with a highly reflective white paint. The light intensity is increased by an additional mirror foil on the front face. The homogenized ligh: reaches the lead glass and the PMTs through holes in the front face.

The light of all three LEDs is also measured by PIN photo diodes mounted on the outside of the plastic cover. The photo diode is read out via hybrid preamplifiers and twisted-pair drivers directly at the Supermodule feeding $40 \mathrm{~m}$ long cables to the shapers and peak-sensing ADCs. A test pulse is distributed to all preamplifiers to monitor the electronics.

In addition, a temperature sensor is sitting close to the photo diode to allow a correction for potential temperature drifts. The sensors are read out via line drivers with scanning ADCs. A schematic layout of the monitoring system electronics is shown in fig. II.C.14.2.

For the readout of the PMTs a new ADC system is currently being developed at the ORNL. The system will use an analog memory unit so that no delay cables are necessary and the ADCs can be mounted directly at the detector. The signals will be amplified by a factor of 8 and both the direct and amplified signals will be digitized with a 10 bit Wilkinson ADC, providing the spectrometer with an effective ADC resolution of 13 bits. 


\section{References for High Energy Heavy-Ion Physics}

[1] A. Shor, in Proceedings of the Second Workshop on Experiments and Detectors for a Relativistic Heavy Ion Collider (RHIC), Hans-Georg Ritter and Asher Shor, Eds., LBL-24604, CONF-870543, p.256.

[2] M. Leitch, E789 collaboration, private communication.

[3] A. Chilingarov et al., Phys. Lett. B83, 136 (1979).

[4] B. Müller and X.-Nv. Wang, Phys. Rev. Lett. 68, 2437 (1992).

E. Shuryak, SUNY-NTG-91-48 (1991). (1979).

[5] T. Akesson et al., Phys. Lett. B152, 411 (1985).

H. Cobbaert et al., Phys. Lett. B191, 456 (1987).

[6] R. Vogt, Atom. Nucl. Data Tabl. 50, 343 (1992).

[7] N. S. Craigie, Phys. Rep. 47,1 (1978).

[8] C. Gerschel and J. Hufner. Nucl. Phys. A544, 513c (1992).

[9] H. Wind, "Principal Component Analysis and its application to track finding", In Formula and Methods in Experimental Data Evaluation, Vol. 3, European Physical Society, 1984

[10] Off-line Computing for RHIC Experiments, Bill Love et al., RHIC Detector Note 8, 1992.

[11] SDC: Technical Design Report, SSCL-SR-1215, 1992.

[12] RHIC Off-line Computing, Study Group Interim. Report, J. Featherly et al., BNL, June 1993.

[13] National Instruments Corporation, Lab VIEW 2 User Manual Jan. 1993 Edition.

[14] This contribution is an update of the proton data analysis reported in last year's Progress Report.

[15] R. Albrecht et al., (WA80-Coll.), Z. Phys. C45 (1990) 529

[16] R. Albrecht et al., (WA80-Coll.), to be subm. to Nucl. Phys. A (1994)

[17] (WA80-Coll.), Nucl. Phys. A544 (1992) 449c

[18] K.H. Kampert et al., (WA80-Coll.), Prog. Part. Nucl. Phys. 30 (1993) 171

[19] D. Brill et al., (KaoS-Coll.), Phys. Rev. Lett. 71 (1993) 336; L.B. Venema et al.,(TAPSColl.) Phys. Rev. Lett. 71 (1993) 835

[20] A. Baldin et al., Yad. Fiz. 18 (1973) 79.; A. Baldin et al., Sovj. Journ. Nucl. Phys. 18 (1973) 41.

[21] A. Baden et al., Nucl. Instr. and Meth. 203 (1982) 189.

[22] G. Young et al., Nucl. Instr. and Meth. A 279 (1989) 503-517.

[23] Proposal for a large acceptance hadron and photon spectrometer, preprint CERN/SPSLC 91-17 (1991) 



\section{The Joint Institute for Heavy Ion Research}

C. R. Bingham [UTK],J. H. Hamilton [Vanderbilt], and R. L. Robinson [ORNL]

The Joint Institute for Heavy Ion Research is a collaborative endeavor between the University of Tennessee, Vanderbilt University, and ORNL. About three-fourths of its funding comes from the State of Tennessee through the Science Alliance at the University of Tennessee. The remainder is provided by Vanderbilt University, DOE, and ORNL. The Joint Institute is housed in two buildings which are adjacent to the Holifield Facility.

It has continued to fulfill its mission as defined in its agreement between the three sponsoring institutes:

"to promote and support heavy-ion research at the Holifield Facility by providing an intellectual center and physical support for researchers who work at the Holifield Facility, to the mutual benefit of sponsors and participants, in the field of heavy ion research."

This is done is three ways: 1) support of workshops and meetings, 2) support of guests, and 3) operation of a dormitory facility.

During FY1992 support was provided for five meetings noted in Table III..0.1 which were attended by about 275 persons. The largest of these was the Workshop on the Production of Intense Radioactive Beams at the Isospin Laboratory, a workshop attended by many representatives from across North America and a few from Europe to discuss techniques for producing and accelerating radioactive ion beams for use in Astrophysics and Nuclear Physics studies. In addition, there was discussion of the exciting physics to be pursued by such a machine. The Workshop on Experimental Apparatus for the Holifield RIB Facility comprised a discussion of details of the experimental program to be pursued at HRIBF, and in particular, the detection equipment and instrumentation needed to pursue this program was discussed. The groundwork was laid to enlist many of the HHIRF users in the design and construction of equipment for the HRIBF. The Vanderbilt Summer Science Collaborative introduced undergraduate students to various scientific endeavors including nuclear structure studies at HRIBF. The symposium New Physics Initiatives with Radioactive Beams was an active one-day discussion of exciting physics studies which will become possible with the startup of radioactive beams. The Symposium on Identical Bands in Nuclear Physics comprised an indepth discussion of this phenomenon in superdeformed and normally deformed nuclei.

Full or partial support was provided to the guests noted in Table III..0.2. The average length of appointment during FY 1993 for the 56 guests was over 3 months. 
Table III..0.1: Meetings sponsored by the JIHIR during FY 1992

\begin{tabular}{llll}
\hline Meeting & Date & Attendees & Chairmen \\
\hline $\begin{array}{l}\text { The Workshop on the } \\
\text { Production of Intense }\end{array}$ & October 7-10,1992 & 125 & J. D. Garrett \\
$\begin{array}{l}\text { nudioactive Beams at } \\
\text { the Isospin Laboratory }\end{array}$ & & & \\
$\begin{array}{l}\text { Workshop on Experimental } \\
\text { Apparatus for the } \\
\text { Holifield RIB Facility }\end{array}$ & October 10-11, 1992 & 46 & R. L. Robinson \\
$\begin{array}{l}\text { Vanderbilt Summer } \\
\begin{array}{l}\text { Science Collaborative } \\
\text { New Physics Initiatives } \\
\text { with Radioactive Beams }\end{array}\end{array}$ & August 11, 1993 16-18, 1993 & 30 & J. H. Hamilton \\
$\begin{array}{l}\text { Symposium on Identical } \\
\text { Bands in Nuclear Physics }\end{array}$ & August 12, 39 & J. D. Garrett \\
\hline
\end{tabular}

The primary effort was in the area of nuclear structure theory with the purpose of maintaining a strong nuclear structure theory effort at ORNL/UT. Thus, we have pledged funds from our state resources to supplement funds from DOE in support of theory pertinent to future measurements at HRIBF. In this fiscal year, we provided half support for a senior nuclear structure theorist (Witek Nazarewicz) and support for a postdoctoral research associate (Javid Sheikh) and an additional $\$ 70,000$ to strengthen this expanding theoretical effort. The new theory effort and the JIHIR will comprise an important component in the study of nuclei near the proton drip line and in the region of astrophysical significance. A significant effort was made this year in the development of radioactive ion beam capability at Oak Ridge, with support of Joe Dellwo and Hermann Wolnik for an extended time and several other experts for shorter visits.

The dormitory was used this yearto house some of the JIHIR visitors and other short-term guests. It provided lodging for 73 guests who stayed for a total of 930 person-nights. 
Table III..0.2: Guest scientists at the Joint Institute for Heavy Ion Research during the period of October 1992 - September 1993

\begin{tabular}{lll}
\hline Name & Institute & $\begin{array}{l}\text { Length of } \\
\text { Appointment }\end{array}$ \\
\hline Sven Aberg & Univ. of Lund (Sweden) & 2 weeks \\
Baha Balantekin & Univ. of Wisconsin-Madison & 1 week \\
Valmir Barbosa & Univ. Federal at Rio de Janeiro (Brazil) & 1 week \\
Frank Berg & Univ. of Giessen (Germany) & 3 days \\
Kumar Bhatt & Univ. of Mississippi & 1 mo. \\
James Blankenship & ORNL & 1 year \\
Efrain Chavez-Lomeli & Nat'l Univ. of Mexico & 3 weeks \\
Yong-Shou Chen & China Inst. of Atomic Energy & 3 mo. \\
Nikola Cindro & Ruder Boskovic Inst. (Croatia) & 5 weeks \\
David Cullen & Univ. of Liverpool & 1 year \\
Joe Dellwo & UT/ORNL & 1 year \\
Jacek Dobaczewski & Warsaw University (Poland) & 1 mo. \\
Raul Donangelo & Inst. de Fisica UFRJ (Brazil) & 1 mo. \\
Jerzy Dudek & Centre de Recherches Nucleaires (France) & 2 weeks \\
Amand Faessler & University of Tubingen (Germany) & 1 week \\
Dsuan Feng & Drexel University & 1 mo. \\
Hubert Flocard & Centre Nat. De La Recherche Sci. (France) & 3 weeks \\
Stefan Frauendorf & NBI (Denmark) & 2 weeks \\
Walter Greiner & University of Frankfurt (Germany) & 2 mo. \\
Gudrun Hagamann & NBI (Denmark) & 2 weeks \\
Ikuko Hamamoto & Lund University (Sweden) & 6 weeks \\
Bruce Harmon & NASA/Marshall Space Flight Center & 1 week \\
Ian Hughes & JET Joint Undertaking (England) & 3 mo. \\
Victor Irby & University of Missouri & 11 mo. \\
Arthur James & University of Liverpool (United Kingdom) & 2 weeks \\
John Klauder & University of Florida & 2 weeks \\
Milorad Korolija & Ruder Boskovic Institute (Croatia) & 1 year \\
\hline & & \\
\hline
\end{tabular}


Table III..0.2: cont. Guest scientists at the Joint Institute for Heavy Ion Research during the period of October 1992 - September 1993

\begin{tabular}{|c|c|c|}
\hline Name & Institute & $\begin{array}{l}\text { Length of } \\
\text { Appointment }\end{array}$ \\
\hline Zhen-Ping Li & Carnegie Mellon Univ. & $1 \mathrm{mo}$. \\
\hline Joachim Maruhn & University of Frankfurt (Germany) & 1 week \\
\hline Francis McGowan & ORNL & 1 year \\
\hline Paul Mueller & University of Illinois & 1 year \\
\hline Witold Nazarewicz & Warsaw University (Poland) & 1 year \\
\hline Yuri Oganessian & JINR ( Russia) & 1 day \\
\hline Erich Ormand & CalTech & 2 days \\
\hline Takaharu Otsuka & Univ. of Tokyo (Japan) & 3 days \\
\hline Alexandrina Petrovic & Univ. of Tubingen (Germany) & 1 week \\
\hline Dieter Rentsch & Univ. of Giessen (Germany) & 3 days \\
\hline Shaheen Rab & Kuwait Inst. for Scientific Research & 1 year \\
\hline John Rasmussen & Lawrence Berkely Lab. & 2 weeks \\
\hline Stanislaw Rohozinski & Warsaw University (Poland) & $1 \mathrm{mo}$. \\
\hline Surender Saini & ORNL & 1 year \\
\hline Marcel Schmorak & ORNL & 1 year \\
\hline Javid Sheikh & Tata Inst. for Fund. Research (India) & $7 \mathrm{mo}$. \\
\hline Janusz Skalski & Soltan Inst. Nuclear Studies (Poland) & $1 \mathrm{mo}$. \\
\hline David Sousa & Eastern Kentucky Univ. & 3 weeks \\
\hline Zdzislaw Szymanski & Warsaw University (Poland) & 2 months \\
\hline Fredrich Thielemann & Harvard University & 1 week \\
\hline S. Umar & Vanderbilt Univ. & $1 \mathrm{mo}$. \\
\hline Michel Vallieres & Drexel University & $1 \mathrm{mo}$. \\
\hline D. Vautherin & Inst. De Physique (France) & 2 weeks \\
\hline Roseli Weàemann & Inst. de Fisica UFRJ (Brazil) & 2 weeks \\
\hline Tomasz Werner & Inst. For Theoretical Phys. & $4 \mathrm{mo}$. \\
\hline Hermann Wollnik & University of Giessen (Germany) & 6 weeks \\
\hline Phillip Woods & Edinburgh Univ. (United Kingdom) & 2 weeks \\
\hline Nobuaki Yoshida & Inst. of Phys. \& Chem. Research (Japan) & $1 \mathrm{mo}$. \\
\hline Tian-Yuan Zhang & Inst. App. Phys. \& Comp. Math (China) & 1 week \\
\hline
\end{tabular}




\section{List of Publications}




\section{IV.A. Papers Published or Ready for Submission}

1. "Search for Population of Superdeformed States in ${ }^{194} \mathrm{~Pb}$ Using ${ }^{194} \mathrm{Bi} \beta^{+}$-Decay," M. A. Stoyer, E. A. Henry, J. A. Becker, R. W. Hoff, A. Kuhnert, D. Manatt, T. F. Wang, J. Breitenhach, M. Jarrio, J. L. Wood, Y. A. Akovali, C. R. Bingham, M. Zhang, P. Joshi, H. K. Carter, J. Kormicki, and P. F. Mantica, Phys. Rev. C47 (1993) 76.

2. "New Results on Superdeformed Bands in $\mathrm{Hg}$ and $\mathrm{Tl}$ Nuclei," M. P. Carpenter, R. V. F. Janssens, Y. Liang, I. G. Bearden, I. Ahmad, M. W. Drigert, U. Garg, R. G. Henry, J. M. Lewis, T. L. Khoo, T. Lauritsen, S. Pilotte, W. Reviol, L. L. Riedinger, F. Soramel, and C. H. Yu, Nuclear Physics A 557 (1993) 57c.

3. "Intruder states at High Spins," L. L. Riedinger, H. Jensen, W. F. Mueller, C.-H. Yu, J.-Y. Zhang, and W. Nazarewicz, Proceedings of International Symposium on Nuclear Physics of Our Times, Sanibel Island, FL, November 16-22, 1992, (World Scientific, 1993) 239.

4. "Production of Slow Single Charged Fragment in $200 \mathrm{GeV} / \mathrm{c}$ Hadron Nucleus Interactions," R. Albrecht, T. C. Awes, C. Baktash, P. Beckmann, F. Berger, R. Bock, G. Claesson, G. Clewing, L. Dragon, A. Eklund, R. L. Ferguson, A. Franz, S. Garpman, R. Glasow, H. A. Gustafsson, H. H. Gutbrod, J. Idh, P. Jacobs, K.-H. Kampert, B. W. Kolb, P. Kristiansson, I. Y. Lee, H. Lohner, I. Lund, F. E. Obenshain, A. Oskarsson, I. Otterlund, T. Peitzmann, S. Persson, F. Plasil, A. M. Poskanzer, M. Purschke, H.-G. Ritter, S. Saini, R. Santo, H. R. Schmidt, T. Siemiarczuk, S. P. Sorensen, K. Steffens, E. Stenlund, D. Stueken, M. L. Tincknell, and G. R. Young, Z. Phys. C 57 (1993) 37.

5. "Emission of Slow Singly Charged Fragments in Relativistic 160-Nucleus Interactions," R. Albrecht, T. C. Awes, C. Baktash, P. Beckmann, F. Berger, R. Bock, G. Claesson, G. Clewing, L. Dragon, A. Eklund, R. L. Ferguson, A. Franz, S. Garpman, R. Glasow, H. A. Gustafsson, H. H. Gutbrod, J. Idh, P. Jacobs, K.-H. Kampert, B. W. Kolb, P. Kristiansson, I. Y. Lee, H. Lohner, I. Lund, F. E. Obenshain, A. Oskarsson, I. Otterlund, T. Peitzmann, S. Persson, F. Plasil, A. M. Poskanzer, M. Purschke, H.-G. Ritter, S. Saini, R. Santo, H. R. Schmidt, T. Siemiarczuk, S. P. Sorensen, K. Steffens, E. Stenlund, D. Stueken, M. L. Tincknell, and G. R. Young, Phys. Lett. B 307 (1993) 269.

6. "Search for Diabolical Pair Transfer in Two-Neutron Transfer Reactions," K. G. Helmer, C. Y. Wu, D. Cline, A. E. Kavka, W. J. Kernan, E. G. Vogt, M. W. Guidry, X. 
L. Han, R. W. Kincaid, X. T. Liu, H. Schechter, J. O. Rasmussen, A. Shihab-Eldin, M. A. Stoyer, and M. L. Halbert, Phys. Rev. C 48(4) (1993) 1879.

7. "Search for Low-Spin Superdeformed States in Nuclei," C. R. Bingham, M. Zhang, J. A. Becker, E. A. Henry, R. W. Hoff, A. Kuhnert, M. A. Stoyer, T. F. Wang, Y. A. Akovali, P. Joshi, T. S. Lam, D. Rupnik, E. F. Zganjar, J. Breitenbach, M. Jarrio, J. L. Wood, H. K. Carter, P. F. Mantica, and J. Kormicki, Nucl. Instru. and Meth. in Physics Research B79 (1993) 309.

8. "Recent Results from the Argonne Fragment Mass Analyzer," C. N. Davids, B. Back, I. G. Bearden, K. Bindra, C. R. Bingham. R. Broda, M. P. Carpenter, W. Chung, P. J. Daly, B. Fornal, Z. W. Grabowski, D. J. Henderson, R. G. Henry, R. V. F. Janssens, T. L. Khoo, T. Lauritsen, Y. Liang, R. H. Mayer, D. M. Moltz, D. Nisius, A. V. Ramayya, J. D. Robertson, F. Scarlassara, F. Soramel, P. Spolaore, K. S. Toth, and W.B. Walters, Proceedings of International Symposium on Nuclear Physics in Our Times, Sanibel Island, FL, November 16-22, 1992, (World Scientific, 1993) 355.

9. "Nuclear Superdeformation and the Supershell Fermion Dynamical Symmetry Model," C.-L. Wu, D. H. Feng, and M. W. Guidry, Annals of Physics 222 (1993) 187.

10. "Some General Constraints on Identical Band Symmetries," M. W. Guidry, M. R. Strayer, C.-L. Wu, and D. H. Feng, Phys. Rev. C 48 (1993) 1739.

11. "Relation of Colective Observables to Nuclear Shapes," J.-Y. Zhang, R. F. Casten, and N. V. Zamfir, Phys. Rev. C48 (1993) R10.

12. "Is Nuclear Structure Really so Simple?," D. S. Brenner, R. F. Casten, W.T. Chou, J.-Y. Zhang, N. V. Zamfir, and K. Heyde, 6th Int. Conf. on Nuclei Far From Stability and 9th Int. Conf. on Atomic Mass and Fundamental Constants, Bernkastel-Kues, Germany, July 19-24, 1992, Inst. Phys. Conf. Ser. 132 (1993) 615.

13. "First Results from the Fragment Mass Analyzer at ATLAS," C. N. Davids, I. Ahmad, B. B. Back, I. G. Bearden, R. R. Betts, K. Bindra, C. R. Bingham, D. J. Blumenthal, R. Broda, M. P. Carpenter, P. Chowdhury, W. Chung, B. Crowell, P. J. Daly, B. Fornal, S. J. Freeman, M. Freer, J. Gehring, Z. W. Grabowski, D. J. Henderson, R. Henry, R. V. F. Janssens, T. Lauritsen, Y. Liang, C. J. Lister, R. H. Mayer, D. M. Moltz, D. Nisius, A. V. Ramayya, J. D. Robertson, F. Scarlassara, P. Spolaore, F. Soramel, and W. B. Walters, 6th Int. Conf. on Nuclei far from Stability and 9th Int. Conf. on Atomic Masses and Fundamental Constants, 
Bernkastel-Kues, Germany, July 19-24, 1992, Inst. Phys. Conf. Ser. 132 (1993) 931.

14. "Tracking Intruder States," L. L. Riedinger, W. F. Mueller, and C.-H. Yu, Proceedings of Int. Conf. on Nucl. Struc. at High Angular Momentum, Ottawa, Canada, May, 1992, AECL 10613 Vol 2 (1993) 339.

15. “Superdeformation in ${ }^{191}$ Tl," S. Pilotte, J. M. Lewis, L. L. Riedinger, C.-H. Yu, M. P. Carpenter, R. V. F. Janssens, T. L. Khoo, T. Lauritsen, Y. Liang, F. Soramel, and I. G. Bearden, Proceedings of Int. Conf. on Nucl. Struc. at High Angular Momentum, Ottawa, Canada, May, 1992, AECL 10613 Vol 2 (1993) 2.

16. "Spectroscopy of ${ }^{96,97,98} \mathrm{Ru}$ : Possible Emergence of Collectivity at $\mathrm{N} \geq 52$," W. Reviol, U. Garg, I. Ahmad, A. Aprahamian, M. P. Carpenter, B. F. Davis, R. V. F. Janssens, T. L. Khoo, T. Lauritsen, Y. Liang, S. Naguleswaran, J. C. Walpe, and D. Ye, Nucl. Phys. A 557 (1993) 391c.

17. "Experiments Using the Argonne Fragment Mass Analyzer," C. N. Davids, B. Back, I. G. Bearden, K. Bindra, C. R. Bingham, R. Broda, M. P. Carpenter, W. Chung, P. J. Daly, B. Fornal, Z. W. Grabowski, D. J. Henderson, R. G. Henry, R. V. F. Janssens, T. L. Khoo, T. Lauritsen, Y. Liang, R. H. Mayer, D. M. Moltz, D. Nisius, A. V. Ramayya, J. D. Robertson, F. Scarlassara, F. Soramel, P. Spolaore, K. S. Toth, and W. B. Walters, Proceedings of the XVI Symposium on Nuclear Physics, Oaxtepec, Mexico, January, 1993.

18. "Research at the Fragment Mass Analyzer at ATLAS," C. N. Davids, B. Back, I. G. Bearden, K. Bindra, C. R. Bingham, R. Broda, M. P. Carpenter, W. Chung, L. Conticchio, P. J. Daly, B. Fornal, M. Freer, Z. W. Grabowski, D. J. Henderson, R. G. Henry, R. V. F. Janssens, C.-L. Jiang, T. L. Khoo, T. Lauritsen, Y. Liang, R. H. Mayer, D. Nisius, A. V. Ramayya, F. Scarlassara, F. Soramel, P. Spolaore, W. B. Walters, A. H. Wuosmaa, and B. E. Zimmerman, Proceedings of the International School on Heavy Ion Physics, Dubna, Russia, May, 1993.

19. "Dynamical Symmetry and Shape Coexistence," M. W. Guidry, Proceedings of International Symposium on Nuclear Physics of Our Times, Sanibel Island, FL, November 16-22, 1992, (World Scientific, 1993) 277.

20. "Spectroscopy of the Superdeformed band in ${ }^{196} \mathrm{~Pb}$, , E. F. Moore, Y. Liang, R. V. F. Janssens, M. P. Carpenter, I. Ahmad, I. G. Bearden, P. J. Daly, M. W. Drigert, B. Fornal, U. Garg, Z. W. Grabowski, H. L. Harrington, R. G. Henry, T. L. Khoo, T. Lauritsen, R. H. Mayer, D. Nissius, W. Reviol, and M. Sferrazza, Phys. Rev. C 48 (1993) 2261. 
21. "Lifetime Measurements in ${ }^{118,120}$ Xe," P. F. Mantica, P. K. Joshi, S. J. Robinson, E. F. Zganjar, R. L. Gill, W. B. Walters, D. Rupnik, H. K. Carter, J. Kormicki, and C. R. Bingham, 6th Int. Conf. on Nuclei far from Stability and 9th Int. Conf. on Atomic Masses and Fundamental Constants, Bernkastel-Kues, Germany, July 19-24, 1992, Inst. Phys. Conf. Ser. 132 (1993) 667.

22. "The $\alpha$-Decay Properties of Light Uranium Isotopes," K. S. Toth, H. J. Kim, J. W. McConnell, C. R. Bingham, and D. C. Sousa, 6th Int. Conf. on Nuclei far from Stability and 9th Int. Conf. on Atomic Masses and Fundamental Constants, Bernkastel-Kues, Germany, July 19-24, 1992, Insi. Phys. Conf. Ser. 132 (1993) 707.

23. "Deformation of ${ }^{187} \mathrm{Tl}$ from Laser Spectroscopy and its Interpretation in the Strutinsky and Particle Plus Rotor Models," H. A. Schuessler, E. C. Benck, F. Buchinger, H. Climura, R. Wyss, C. R. Bingham, H. K. Carter, and J. Rikovska, 6th Int. Conf. on Nuclei far from Stability and 9th Int. Conf. on Atomic Masses and Fundamental Constants, Bernkastel-Kues, Germany, July 19-24, 1992, Inst. Phys. Conf. Ser. 132 (1993) 221.

24. "Symmetry and the Origin of Nuclear Deformation," M. W. Guidry and C. L. Wu, Int. J. Mod. Physics E 2 (1993) 17.

25. "Investigation of Proton-Rich Platinum and Mercury Isotopes with the Fragment Mass Analyzer at ATLAS and the Isotope Separator at UNISOR," K. S. Toth, C. N. Davids, Y. A. Akovali, B. B. Back, K. Bindra, C. R. Bingham, H. K. Carter, W. Chung, Y. Hatsukawa, D. J. Henderson, T. Lauritsen, P. F. Mantica, D. M. Moltz, A. V. Ramayya, J. D. Robertson, and W. B. Walters, 6th Int. Conf. on Nuclei far from Stability and 9th Int. Conf. on Atomic Masses and Fundamental Constants, Bernkastel-Kues, Germany, July 19-24, 1992, Inst. Phys. Conf. Ser. 132 (1993) 589.

26. "Lifetime of the $\mathrm{O}_{2}^{+}$Configuration in ${ }^{186} \mathrm{Hg}$ and ${ }^{188} \mathrm{Hg}$," P. K. Joshi, S. J. Robinson, P. F. Mantica, E. F. Zganjar, D. Rupnik, R. L. Gill, W. B. Walters, H. K. Carter, C. R. Bingham, J. Kormicki, A. V. Ramayya, and W. C. Ma, 6th Int. Conf. on Nuclei far from Stability and 9th Int. Conf. on Atomic Masses and Fundamental Constants, Bernkastel-Kues, Germany, July 19-24, 1992, Inst. Phys. Conf. Ser. 132 (1993) 727.

27. "New Mass Differences and $\alpha$-Decay Rates for Au and Pt Isotopes," C. R. Bingham, Y. A. Akovali, H. K. Carter, W. D. Hamilton, M. M. Jarrio, M. B. Kassim, J. Kormicki, J. Schwarzenberg, K. S. Toth, and M. Zhang, 6th Int. Conf. on Nuclei far from Stability and 9th Int. Conf. on Atomic Masses and Fundamental 
Constants, Bernkastel-Kues, Germany, July 19-24, 1992, Inst. Phys. Conf. Ser. 132 (1993) 735.

28. "The Polarizing Effect of SO(6) Symmetry in the Fermion Dynamical Symmetry Model," L.-A. Wu, D.-L. Wu, M. W. Guidry, and D. H. Feng, Nucl. Phys. A 565 (1993) 455.

29. "The Fermion Dynamical Symmetry Model," C. L. Wu, D. H. Feng, and M. W. Guidry, Advances in Nuclear Physics 21 (1994) 227.

30. "Lifetimes of the $0_{2}^{+}$Configuration in ${ }^{186} \mathrm{Hg}$ and ${ }^{188} \mathrm{Hg}$," P. K. Joshi, E. F. Zganjar, D. Rupnik, S. J. Robinson, P. F. Mantica, H. K. Carter, R. L. Gill, W. B. Walters, C. R. Bingham, J. Kormicki, A. V. Ramayya, W. C. Ma, and J. H. Hamilton, accepted for publication, Int'l Journal of Mod. Phys. E: Nuclear Physics.

31. "Superdeformation in ${ }^{191}$ Tl," S. Pilotte, C.-H. Yu, H.-Q. Jin, J. M. Lewis, L. L. Riedinger, M. P. Carpenter, R. V. F. Janssens, T. L. Khoo, T. Lauritsen, Y. Liang, F. Soramel, I. G. Bearden, C. Baktash, J. D. Garrett, N. R. Johnson, I. Y. Lee, and F. K. McGowan, accepted for publication, Physical Review C.

32. "New Signature for Phase/Shape Transitional Regions," N. V. Zamfir, R. F. Casten, J.-Y. Zhang, and D. S. Brenner, 8th International Symposium on Capture Gamma Ray Spectroscopy and Related Topics, Fribourg, Switzerland, Sept. 2024,1993 , to be published.

33. "Some Collective Observables in Nuclear Motion," Jing-ye Zhang, accepted for publication, High Energy Physics and Nuclear Physics (China).

34. "Observation of New Neutron-Rich Nuclide ${ }^{208} \mathrm{Hg}$," L. Zhang, G.-M. Jin, J.-H. Zhao, W.-F. Yang, Y.-F. Yang, Z.-Z. Zhao, J.-W. Zheng, X.-R. Sun, J.-C. Wang, Z.-W. Li, Z. Qin, G.-H. Guo, Y.-X. Lu, Jan Zylioz, and J.-Y. Zhang, accepted for publication, Phys. Rev. C.

35. "Prolate Collectivity in ${ }^{187} \mathrm{Tl}$," W. Reviol, L. L. Riedinger, J.-Y. Zhang, W. F. Mueller, C. R. Bingham, B. E. Zimmerman, R. V. F. Janssens, M. P. Carpenter, I. Ahmad, I. G. Bearden, R. G. Henry, T. L. Khoo, T. Lauritsen, and Y. Liang, accepted for publication, Phys. Rev. C.

36. "Measurements of Transverse Energy and Foward Energy in High Energy Nuclear Collisions," S. P. Sorensen, Proc. 2nd Int. Conf. on the Physics and Astrophysics of the Quark-Gluon Plasma, Calcutta, India, January, 1993, to be published.

37. " $\alpha$-Decay Rates for ${ }^{181-186} \mathrm{Au}$ and ${ }^{181-185} \mathrm{Pt}$ Isotopes," C. R. Bingham, M. B. 
Kassim, M. Zhang, Y. A. Akovali, K. S. Toth, W. D. Hamilton, H. K. Carter, J. Kormicki, J. Von Schwarzenberg, and M. M. Jarrio, submitted to Phys. Rev. C.

38. "Anomalous Population of Excited States in Transfer Reactions with Heavy Ions," C. Y. Wu, X. T. Liu, M. W. Guidry, C. R. Bingham, D. Cline, T. Czosnyka, A. E. Kavka, W. J. Kernan, and S. P. Sorensen, submitted to Phys. Lett. B.

39. "Possible Evidence for a Nuclear Josephson Effect," M. W. Guidry, X. T. Liu, C. Y. Wu, C. R. Bingham, D. Cline, T. Czosnyka, A. E. Kavka, and W. J. Kernan, submitted to Phys. Rev. C.

40. "Probabilities for the Direct Population of High-Spin States in Transfer Reactions," X. T. Liu, C. Y. Wu, M. W. Guidry, D. Cline, T. Czosnyka, A. E. Kavka, W. J. Kernan, R. W. Kincaid, and S. P. Sorensen, submitted to Phys. Rev. C.

41. "Effects of Intruder States in Ir Nuclei," H. Q. Jin, C. R. Bingham, M. P. Carpenter, V. P. Janzen, L. L. Riedinger, C. H. Yu, L. Zhou, P. B. Semmes, J.-Y. Zhang, M. A. Riley, C. Baktash, M. L. Halbert, N. R. Johnson, I. Y. Lee, and F. K. McGowan, submitted to Physical Review $C$.

42. "Identical Transitions between Superdeformed and Normally Deformed Bands and the Cancellation Picture," Jing-ye Zhang, submitted to Phys. Lett. B.

43. "Signature Splitting in Nuclear Rotational Bands: I. Neutron $i_{13 / 2}$ Systematics," W. F. Mueller, H. J. Jensen, W. Reviol, L. L. Riedinger, C.-H. Yu, J.-Y. Zhang, W. Nazarewicz, and R. Wyss, submitted to Phys. Rev. C.

44. "Inclusive Photon Transverse Momentum Distributions from Ultrarelativistic Heavy-Ion Collisions at $60 \mathrm{~A}$ and $200 \mathrm{~A} \mathrm{GeV/c}$ at the CERN-SPS," R. Albrecht, T. C. Awes, C. Baktash, P. Beckmann, F. Berger, R. Bock, G. Claesson, G. Clewing, L. Dragon, A. Eklund, R. L. Ferguson, A. Franz, S. Garpman, R. Glasow, H. A. Gustafsson, H. H. Gutbrod, J. Idh, P. Jacobs, K.-H. Kampert, B. W. Kolb, P. Kristiansson, I. Y. Lee, H. Lohner, I. Lund, F. E. Obenshain, A. Oskarsson, I. Otterlund, T. Peitzmann, S. Persson, F. Plasil, A. M. Poskanzer, M. Purschke, H.-G. Ritter, S. Saini, R. Santo, H. R. Schmidt, T. Siemiarczuk, S. P. Sorensen, K. Steffens, E. Stenlund, D. Stueken, M. L. Tincknell, and G. R. Young, submitted to Z. Phys. C.

45. "Intermittency and Correlations in $200 \mathrm{GeV} /$ nucleon S+S and S+Au Collisions," R. Albrecht, A. Antonenko, T. C. Awes, F. Berger, M. A. Bloomer, D. Bock, R. Bock, G. Claesson, G. Clewing, L. Dragon, A. Eklund, S. Fokin, A. Franz, S. Garpman, R. Glasow, H. A. Gustafsson, H. H. Gutbrod, M. Hartig, X. He, G. Holker, J. Idh, M. Ippolitov, P. Jacobs, K.-H. Kampert, K. Karadjev, B. W. Kolb, A. Lebedev, H. Lohner, I. Lund, V. manko, S. Nikolaev, J. Nystrand, F. E. Obenshain, A. Oskarsson, 
I. Otterlund, T. Peitzmann, F. Plasil, A. M. Poskanzer, M. Purschke, H.-G. Ritter, B. Roters, S. Saini, R. Santo, H. R. Schmidt, K. Soderstrom, S. P. Sorensen, K. Steffens, P. Steinhauser, E. Stenlund, D. Stueken, D. Stuken, and G. R. Young, submitted to Phys. Rev. C.

46. "Calorimeter/Absorber Optimization for a RHIC Dimuon Experiment," S. H. Aronson, M. J. Murtagh, M. Starks, X. T. Liu, G. A. Petitt, Ziyang Zhang, L. A. Ewell, J. C. Hill, F. K. Wohn, J. B. Costales, M. N. Namboodiri, T, C. Sangster, J. H. Thomas, A. Gavron, L. Waters, W. L. Kehoe, S. G. Steadman, T. C. Awes, Hee Kim, F. E. Obenshain, S. Saini, M. L. Tinkcnell, G. R. Young, J. Chang, S.-Y. Fung, J. H. Kang, Xiaochun He, J. Kreke, S. P. Sorensen, E. W. Cornell, and C. F. Maguire, submitted to Nucl. Instr. Meth.

\section{IV.B. Abstracts at Meetings}

1. "On-Line Laser Spectroscopy of the Very Neutron Deficient Thallium Isotopes," H. A. Schuessler, et. al., Bull. Am. Phys. Soc. 38 (1993) 946.

2. "Proton Radioactivities ${ }^{146,147}$ Tm," C. N. Davids, et. al. , Bull. Am. Phys. Soc. 38 (1993) 1818.

3. "Decay of Mass-Separated ${ }^{114}$ Te," B. E. Zimmerman, W. B. Walters, P. F. Mantica, H. K. Carter, and J. Kormicki, Bull. Am. Phys. Soc. 38(9) (1993) .

4. "Lifetime Measurements in ${ }^{181}$ Ir and ${ }^{187} \mathrm{Au}$," J. C. Walpe, U. Garg, S. Naguleswaran, Q.-W. Ren, W. Reviol, L. L. Riedinger, X.-Z. Wang, R. V. F. Janssens, I. Ahmad, I. G. Bearden, M. P. Carpenter, R. G. Henry, T. L. Khoo, and T. Lauritsen, Bull. Am. Phys. Soc. 38(9) (1993).

5. "High Spin Studies in ${ }^{187}$ Tl," W. Reviol, L. L. Riedinger, W. F. Mueller, C. R. Bingham, B. E. Zimmerman, J.-Y. Zhang, R. V. F. Janssens, M. P. Carpenter, T. L. Khoo, T. Lauritsen, Y. Liang, R. G. Henry, I. Ahmad, and I. G. Bearden, Bull. Am. Phys. Soc. 38(9) (1993) 1819.

6. "Spectroscopic Studies of the Superdeformed band in ${ }^{196} \mathrm{~Pb}$," E. F. Moore, H. L. Harrington, Y. Liang, R. V. F. Janssens, M. P. Carpenter, I. Ahmad, R. G. Henry, T. L. Khoo, T. Lauritsen, W. Reviol, U. garg, M. W. Drigert, I. G. Bearden, P. J. Daly, B. Fornal, Z. W. Grabowski, R. H. Mayer, and D. Nissius, Bull. Am. Phys. Soc. 38(9) (1993). 
7. "Calculations of Signature Splitting for Dy, Er, Yb Nuclei," W. F. Mueller, H. J. Jensen, W. Reviol, L. L. Riedinger, C.-H. Yu, J.-Y. Zhang, and W. Nazarewicz, Bull. Am. Phys. Soc. 38(9) (1993).

\section{IV.C. Invited Talks}

\section{R. Bingham}

"The Study of Nuclei at and beyond the Proton Drip Line," Int'l Workshop on the Latest Topics in Nuclear Structure, Beijing, PRC, October 24-28, 1993.

"Physics to be Explored with Exotic Beams at the Holifield Radioactive Beam Facility," Int'l Workshop on the Latest Topics in Nuclear Structure, Beijing, PRC, October 24-28, 1993.

\section{W. Guidry}

"Lectures on Nuclear Astrophysics and Radioactive Ion Beam Physics," Tianjin Summer School on Astrophysics, Tianjin, China, september, 1993.

\section{L. Riedinger}

"Use of Signature Splitting for Classification of Multitudes of Rotational Bands," Workshop on Nuclear Spectroscopy at the New Arrays, Lund, Sweden, June 21-22, 1993.

\section{S. P. Sorensen}

"Measurements of Transverse Energy and Foward Energy in High Energy Nuclear Collisions," The 2nd Int. Conf. on the Physics and Astrophysics of the Quark-Gluon Plasma, Calcutta, India, January, 1993.

\section{IV.D. Colloquia}

\section{R. Bingham}

"Study of Particle Emission near the Proton Drip Line," Invited seminar, Institute of Modern Physics, Lanzhou, PRC, October 18, 1993. 
"Study of Nuclei Far From Stability at HHIRF and Conversion to a Radioactive Ion Beam Facility," Invited seminar, Institute of Modern Physics, Lanzhou, PRC, October 19, 1993.

"Search for Low-Spin Low-Energy Superdeformed States in Nuclei," Invited seminar, Institute of Modern Physics, Lanzhou, PRC, October 21, 1993.

\section{L. Riedinger}

"Importance of Intruder Orbitals in the Spectroscopy of High-Spin States at Normal and Super Deformations," Uppsala University, Sweden, June 24, 1993.

\section{W. Reviol}

"Prolate Collectivity in ${ }^{187} \mathrm{Tl}$," Nuclear Physics Lunch at ORNL, Oak Ridge, TN, September 7, 1993.

B. E. Zimmerman

"Report on the Search for $\mathrm{I}=0^{+}$Superdeformed Isomers in non-actinide Nuclides," Given at Indiana University Cyclotron Facility, IN, October, 1993.

\section{IV.E. Nuclear Physics Group Seminars}

"A Systematic Description of the Xe-Ba-Ce Region in IBM," X.W. Pan (Drexel University), February 24, 1993.

"Report on the FMAWorkshop at Argonne," C.R Bingham (UTK), March 3, 1993.

"Pair Correlations in Rapidly-Rotating Nuclei," J.D. Garrett (ORNL), March 10, 1993.

"Proton-Neutron Multiplets in odd-odd Sb Nuclei: the Decay of ${ }^{114}$ Te," B.E. Zimmerman (UTK), March 17, 1993.

“Octupole Deformations in Pr Nuclei," P. Mantica (ORNL), March 31, 1993.

"The Shape of Quantal Droplets: Alkali Atom Droplets Compared with Atomic Nuclei," S. Frauendorf (Rossendorf), April 21, 1993.

"Shape-Coexistence in odd- $A$ Pb," M. Zhang (UTK), April 28, 1993. 
"X-ray Yields for the Superdeformed States in ${ }^{193} \mathrm{Hg}, "$ D.M. Cullen (ORNL), May $5,1993$.

"Early Experiments with RIB at ORNL: Plans, Techniques and Instruments," C. Baktash (ORNL), May 12, 1993.

"Alpha-Decay Studies in even-A Pb," J. Richards (UTK), May 18, 1993.

"Interplay of Single Particle and Collective Motion in Po Nuclei," H.Q. Jin (Rutgers University), May 19, 1993.

"Search for Co-existing Rotational Bands in ${ }^{187}$ Tl," W. Reviol (UTK), May 26, 1993.

"Dramatic Deformation Driving Effect of Rotating Quasiparticle Orbits at Exotic Isospin," Y. Chen (Bejing, China), August 9, 1993.

"The Relation of Collective Observables to Nuclear Shapes," J.Y. Zhang (UTK), September 22, 1993.

"Exotic Nuclei in a Relativistic Framework," J. Sheikh (ORNL), September 29, 1993.

"The UNISOR Picosecond Lifetime Measurement System," P.K. Joshi (LSU), October 6, 1993.

"Signature Splitting in even-odd Rare Earth Nuclei," W.F. Mueller (UTK), October 13, 1993. 


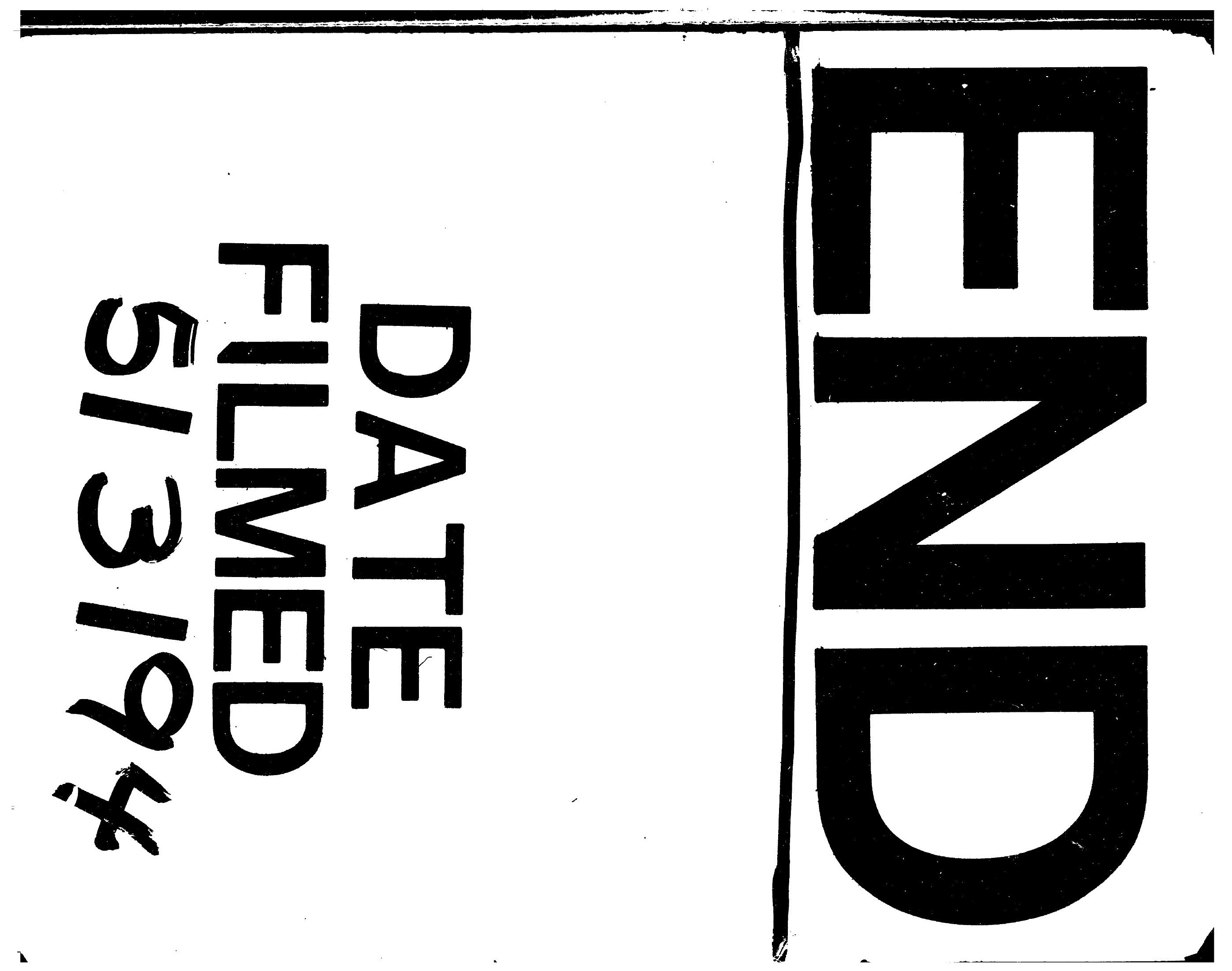


